\title{
U.S.
}

A Preliminary Analysis of Dose Rates Associated with IIER CVCS Equipment/ Area Logation 
This report was prepared as an account of work sponsored by an agency of the United States Government. Neither the United States Government nor any agency thereof, nor any of their employees, makes any warranty, express or implied, or assumes any legal liability or responsibility for the accuracy, completeness, or usefulness of any information, apparatus, product, or process disclosed, or represents that its use would not infringe privately owned rights. Reference herein to any specific commercial product, process, or service by trade name, trademark, manufacturer, or otherwise, does not necessarily constitute or imply its endorsement, recommendation, or favoring by the United States Government or any agency thereof. The views and opinions of authors expressed herein do not necessarily state or reflect those of the United States Government or any agency thereof. 


\title{
A PRELIMINARY ANALYSIS OF DOSE RATES ASSOCIATED WITH THE ITER CVCS EQUIPMENT/AREA LOCATION
}

\author{
Edward D. Blakeman \\ Dan Ilas \\ Technical Project Officer - Andrei Petrov, PhD \\ Tokamak Cooling Water System \\ ORNL US ITER Office
}

Date Published: February 2012

Prepared by

OAK RIDGE NATIONAL LABORATORY

Oak Ridge, Tennessee 37831-6283

managed by

UT-BATTELLE, LLC

for the

U.S. DEPARTMENT OF ENERGY

under contract DE-AC05-00OR22725 



\section{A PRELIMINARY ANALYSIS OF DOSE RATES ASSOCIATED WITH THE ITER CVCS EQUIPMENTIAREA LOCATION}

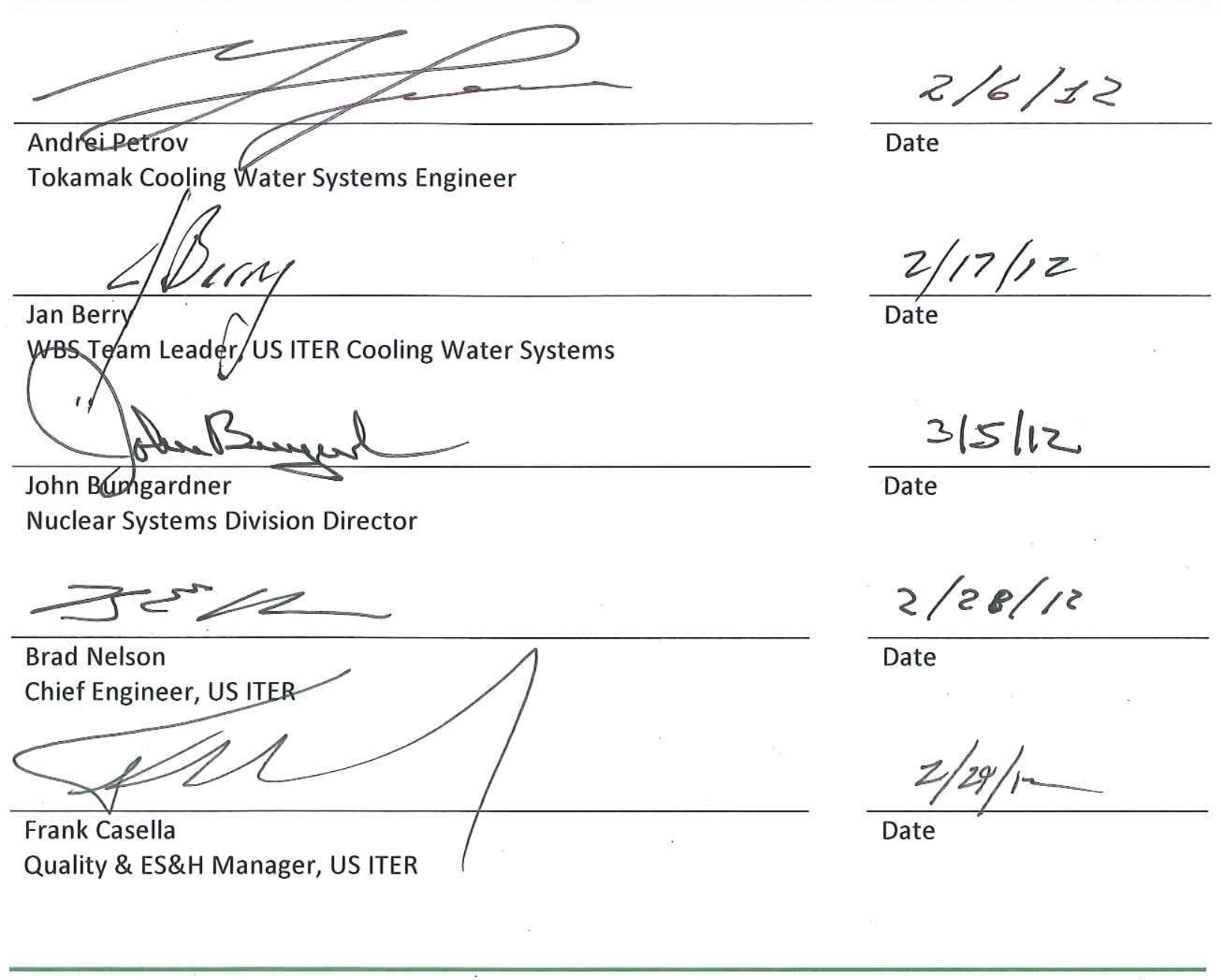





\section{REVISION RECORD}

\begin{tabular}{ccl}
\hline Revision & Date & Description of Changes \\
\hline 00 & $2 / 17 / 2012$ & Original \\
& & \\
\hline
\end{tabular}





\section{TABLE OF CONTENTS}

Page

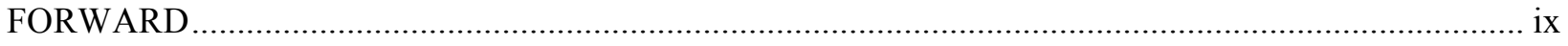

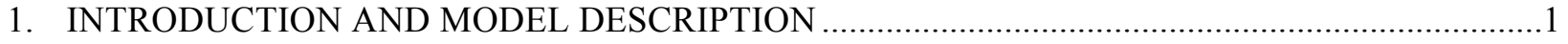

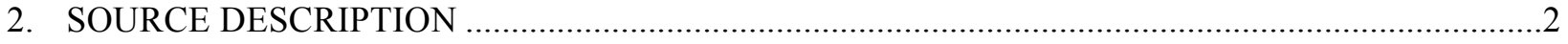

3. DOSE RATE CALCULATION OUTSIDE WALLS AND CEILING ….........................................

4. CALCULATION AT LOCATIONS INSIDE THE FACILITY FOR STEADY-STATE OPERATION (AT SHUtDOWN), AND FOR 2 AND 10 DAYS AFTER SHUTDOWN ...................4

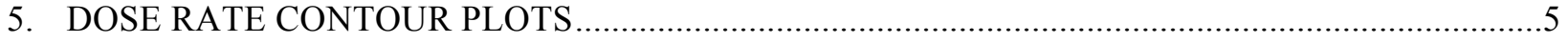

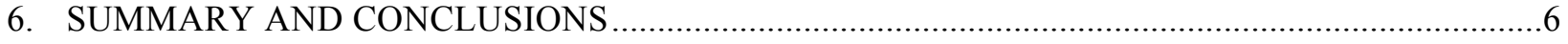

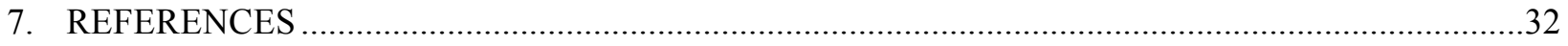

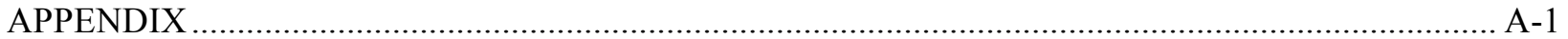





\section{FORWARD}

The report is a revision of an earlier informal report issued on October 10, 2011. It contains results from an updated model in which the walls and ceiling shielding were changed to Barytes concrete, and from a modified model in which particle scattering and shielding for internal dose tallies were eliminated. Also, additional dose rate tally cells at $5 \mathrm{~cm}$ from each component on both horizontal and vertical axes were included. Although most results from the earlier analysis are included in this revised report, the primary emphasis is on the updated model results. In general, descriptions of the model apply to both the earlier and the updated model, except where differences are pointed out. 



\section{INTRODUCTION AND MODEL DESCRIPTION}

A preliminary analysis of the ITER Chemical and Volume Control System (CVCS) Area was performed to assess dose rates outside the walls and ceiling of the facility after 1.5 years of operation at shutdown, 2 days, and 10 days after shutdown. For this purpose a simplified Monte Carlo computer model was developed using the MCNP (MCNP5 Ver. 1.51) [1] code. Two components are included: the smaller filter tank and the larger ion exchanger. These pieces of equipment are associated with the Integrated Blanket ELM Divertor Primary Heat Transfer System, which will have the largest dose rates associated with activated corrosion products during operation in comparison with other systems [2]. The ion exchanger contained two source regions, a 1.2-m-thick resin bed above a $0.55 \mathrm{~m}$-thick skirt, and a 0.8 -m-thick water region. The filter constituted an additional source. Thus the model consisted of three sources (filter, resin, water), homogeneously distributed within the appropriate source regions. However, much of the results (that address individual isotopes) are presented with the two sources in the ion exchanger combined. In these cases the sources are referred to as the "ion exchanger source" and the "filter source." Dimensions for the facility and components, as well as source isotopes and strengths, and material densities, were supplied by US ITER (see Fig.1 and Appendix). Because of its simplification, the model does not contain pipes. Consequently, radiation streaming through pipe penetrations, radiation emanating from the pipes, and shielding from the pipes were not considered in this analysis. Dose rates on the outside of two walls and the ceiling were calculated. The two walls are labeled as the "long" wall (aligned with the $\mathrm{X}$-axis) and the "short" wall (aligned with the Y-axis). These walls and ceiling were nominally set to 30 -cm-thick concrete. In the original analysis, standard concrete [3] (2.3 g/cc density) was used. In the updated analysis standard concrete in these regions was replaced with Barytes concrete [1] (3.35 g/cc density). Compositions of both concrete types by element weight percent are as follows:

Standard concrete: $\mathrm{O}-53.2 \%, \mathrm{Si}-33.7 \%, \mathrm{Ca}-4.4 \%, \mathrm{Al}-3.4 \%, \mathrm{Na}-2.9 \%, \mathrm{Fe}-1.4 \%, \mathrm{H}-1 \%$

Barytes concrete: $\mathrm{Ba}-46.34 \%, \mathrm{O}-31.1622 \%, \mathrm{~S}-10.7858 \%, \mathrm{Ca}-5.0194 \%, \mathrm{Fe}-4.7505 \%$ $\mathrm{Al}-4.138 \%, \mathrm{Mg}-1.195 \%, \mathrm{Si}-1.0457 \%, \mathrm{H}-0.3585 \%$

Except where noted, the following descriptions apply to the original and updated models.

In addition to the shielding walls/ceiling, a floor and an additional wall opposite the long wall were added for photon scattering contributions. These were both 10 -cm-thick, standard concrete structures. Other components (tanks, pipes, etc.), that were not included in the model, would potentially add additional scattering and shielding. Possibly these additional effects will be addressed in a later, moredetailed analysis. The room was $29.6 \mathrm{~m}$ in length (X-axis), but was limited to $15 \mathrm{~m}$ in the model. The inside width ( $\mathrm{Y}$ axis) and height ( $\mathrm{Z}$ axis) were $4 \mathrm{~m}$ and $3.4 \mathrm{~m}$, respectively. The origin for the model was located inside the room at the corner opposite the long wall and adjacent to the short wall at the floor level (shown in Fig. 2). The room was filled with air at standard temperature and pressure.

Outer dimensions of the filter and ion exchanger were as follows:

Filter: $\quad$ diameter $86.4 \mathrm{~cm}$, height: $116.8 \mathrm{~cm}$

Ion exchanger: diameter $250 \mathrm{~cm}$, height: $255 \mathrm{~cm}$

The stainless steel (SS304) wall thicknesses for the ion exchanger and filter were $2.2 \mathrm{~cm}$ and $0.8 \mathrm{~cm}$, respectively. The axial center of the filter was located $140 \mathrm{~cm}$ from the short wall and $100 \mathrm{~cm}$ from the long wall (outer surface). The axial center of the ion exchanger was located $440 \mathrm{~cm}$ from the short wall and $250 \mathrm{~cm}$ from the long wall (inner surface). 
The resin was assumed to be a homogeneous mixture of equal atom density fractions of hydrogen and carbon $^{*}$ at a specified density of $1.136 \mathrm{~g} / \mathrm{cc}$. The filter material was assumed to be homogeneous carbon at a specified density of $1.8 \mathrm{~g} / \mathrm{cc}$. If the filter media were stainless steel and the accumulated activity were the same, the dose rate outside the filter would be lower, provided the density of the stainless steel sintered filter material is significantly higher than $1.8 \mathrm{~g} / \mathrm{cc}$. The densities of the water and air were assumed to be $1.0 \mathrm{~g} / \mathrm{cc}$ and $1.096 \mathrm{E}-3 \mathrm{~g} / \mathrm{cc}$, respectively.

The model included 10 small volumes placed outside the model adjacent to the wall at locations where the dose rates were expected to be highest and which showed to a degree the fall-off of the dose rate with distance along a particular wall or ceiling. These "tally cells" are shown in Fig. 2 and are described in Table 1. Each cell had dimensions of $50 \mathrm{~cm} \times 50 \mathrm{~cm} \times 1 \mathrm{~cm}$ and was oriented so that the 1 -cm-thick dimension was perpendicular to the wall against which the cell was located.

Also, smaller tally volumes were placed at $5 \mathrm{~cm}$ (updated model), $30 \mathrm{~cm}, 50 \mathrm{~cm}$, and $100 \mathrm{~cm}$ from each source, both radially from the outside of each component at the height of each source mid-plane, and vertically, along the axial centerline, above the ion-exchanger and below the filter. (For the ion exchanger, the vertical distance was limited to $75 \mathrm{~cm}$ because of interference from the ceiling.) These tally regions can be seen in Fig. $3^{\dagger}$. Each cell had dimensions of $5 \mathrm{~cm} \times 5 \mathrm{~cm} \times 1 \mathrm{~cm}$, oriented so that the 1-cm-thick dimension was perpendicular to the $\mathrm{X}, \mathrm{Y}$ or $\mathrm{Z}$ axis.

Gamma dose rates were calculated using two sets of photon flux-to-dose conversion factors: 1) ANSI/ANS-6.1.1-1977 and 2) ICRP-2, both from Appendix H of Reference [1]. In general, values from the ANSI set were somewhat higher and judged to be more conservative. Therefore, the ANSI results are reported in this analysis.

The limiting personnel dose rate inside and outside the facility is $0.1 \mathrm{mSv} / \mathrm{h}(100 \mu \mathrm{Sv} / \mathrm{h})$ [4].

\section{SOURCE DESCRIPTION}

The sources provided were of the following strengths (GBq) [Appendix]:

$\begin{array}{lr}\text { Filter: } & 728 \\ \text { Ion exchanger (resin): } & 7283 \\ \text { Ion exchanger (water): } & 632\end{array}$

Gamma-emitting isotopes included Fe-59, Mn-54, Fe-55, Co-58, Co-60, Cr-51, Mn-56, and Cu-64. Gamma energy lines and their production rates were obtained from a database [5,6]. Energies of the lines varied from 126 to $3369.6 \mathrm{KeV}$. Table 2 shows the sources and spreadsheet used to calculate the isotopic source strengths.

Some general observations about the sources as shown in Table 2 can be made. The filter and ion exchanger resin relative isotopic source contributions are nearly identical; however the resin source activity is $\sim 10$ times greater. For each source, $\sim 97.2 \%$ of the activity is from $\mathrm{Cu}-64, \sim 2.3 \%$ from $\mathrm{Mn}-56$, and the remainder from 6 other isotopes. Thus, $\mathrm{Cu}-64$ and $\mathrm{Mn}-56$ were expected to dominate calculated results for these two sources. Although the activity from the $\mathrm{Cu}-64$ is 42 times that from $\mathrm{Mn}-56$, the

\footnotetext{
${ }^{*}$ The formulas for polystyrene $\left(\mathrm{C}_{8} \mathrm{H}_{8}\right) \mathrm{n}$ and divinylbensene $\left(\mathrm{C}_{10} \mathrm{H}_{10}\right)$ both have equal parts (by atom) of hydrogen and carbon.

${ }^{\dagger}$ The tally cells at $5 \mathrm{~cm}$ from the filter and ion exchanger surfaces were added in the updated model and are not shown in Fig. 3 (and would be difficult to discern were they shown).
} 
higher energies and/or higher \%-fractions for three of the lines $(846.754,1810.72$, and $2113.05 \mathrm{KeV})$ from Mn-56 should compensate for the difference in activity levels (as is shown in sections to follow). For the water source, the contribution from the Mn-56 should dominate since the activity of the $\mathrm{Cu}-64$ is only 13 times as great as that from the Mn-56. Contributions from the remaining isotopes should be very small and likely negligible for the water source alone. However, for the isotopic calculations, the resin and water sources are combined for convenience. Thus, these effects for the water source alone were not studied.

\section{DOSE RATE CALCULATION OUTSIDE WALLS AND CEILING}

The highest dose rate contributions were expected to come from tallies $1,4,5,6,7$, and 10, which are either above or across from one of the components.

Calculated values for the 10 tally regions are shown for each source and for the total in Table 3 . Results from the original model are shown for 30-cm-thck and $15-\mathrm{cm}$-thick standard concrete walls and ceilings. Results from the updated model are shown for 30-cm-thick Barytes concrete walls and ceilings. Monte Carlo statistical errors are not shown but are typically $<1 \%$. Exceptions are for the resin source to the ceiling tallies, where the errors are typically $\sim 3 \%$ or less. Also, the error for tally $\# 2$ for the filter source was somewhat high for the Barytes shielding, $\sim 12 \%$, and for the $30-\mathrm{cm}$-thick regular concrete shielding, $\sim 4 \%$, because of the distance of that tally from the source and the large distance that particles would need to travel diagonally from the filter to arrive at that location. From Table 3 it is seen that for the $30-\mathrm{cm}-$ thick, standard concrete walls and ceiling, the largest dose rate is $\sim 0.13 \mathrm{mSv} / \mathrm{h}$ at location 10 (directly above filter transverse center), the second largest is $\sim 0.1 \mathrm{mSv} / \mathrm{h}$ at location 4 which is directly across the long wall, also from the filter vertical center. The third largest is $\sim 0.06 \mathrm{mSv} / \mathrm{h}$, which is at location 1 (directly across the long wall at the resin vertical center). Thus, most dose rate values are below the $0.1 \mathrm{mSv} / \mathrm{h}$ limit for the 30 -cm-thick concrete walls and ceiling.

For the half-thickness $(15 \mathrm{~cm})$ standard concrete wall, the three largest dose rates are at the same locations as above $(10,4$, and 1$)$, and are $\sim 1.1, \sim 0.8$, and $\sim 0.4 \mathrm{mSv} / \mathrm{h}$, respectively. By reducing the wall/ceiling thickness by a factor of 2 , the dose rate is increased by about an order of magnitude. All of the results, with the exception of tally cell \#2, which is located further from the sources, are above the $0.1 \mathrm{mSv} / \mathrm{h}$ limit; this suggests that at some distance (X-Axis), e.g., $400 \mathrm{~cm}$ from the ion chamber center, standard concrete can be used for the long wall and ceiling and the thickness can be reduced to a smaller than nominal $(30 \mathrm{~cm})$ value.

For the 30-cm-thick Barytes concrete walls/ceiling (calculated with updated model), the three largest dose rates are also at locations 10,4 , and 1 , and are $\sim 0.019, \sim 0.015$, and $\sim 0.011 \mathrm{mSv} / \mathrm{h}$, respectively. These results are all well below the $0.1 \mathrm{mSv} / \mathrm{h}$ limit and the other 7 results are more than an order-of- magnitude below the limit.

From Table 3 it can be seen that the contribution of the filter to the dose rates at locations 4,6 , and 10 (long wall, short wall, and ceiling, respectively, close to the filter) is dominant; the contribution from the resin source at long wall locations 1,2 , and 3 , and the short wall position 5 is dominant; the contribution from the water at ceiling positions 7, 8, and 9 (due to the fact that the water is above the resin and shields its effects). If we consider the resin and water as combined sources, then the ion exchanger is dominant at all positions except 4,6 , and 10 . These observations are consistent for all the wall/ceiling configurations shown in Table 3.

Calculations based on individual isotopic sources were also performed with the 30 -cm-thick shielding walls and ceiling using regular (original model) and Barytes (updated model) concrete. This approach 
enabled the evaluation of contributions from each isotope and the calculation of "conversion factors", i.e., dose rate divided by the activity, for each isotope. Using these factors, the dose rate can be recalculated easily at specified locations for different isotopic mixtures. For these calculations, and for calculations in the next section, the resin and water sources were combined into a single source, thus simplifying the sources to "filter" and "ion exchanger". Results for each isotope at each of the 10 external locations shown in Fig. 2 are shown in Tables 4 through 7. Dose rates and conversion factors for the regular concrete walls and ceiling are shown in Tables 4 and 5, respectively. Equivalent values for the Barytes concrete walls and ceiling are shown in Tables 6 and 7. Total dose rate values for each source and for the combined sources are shown in the bottom rows of the sub-tables in Tables 4 and 6 . Combined dose rates in Tables 4 and 6 are equivalent to those in Table 3. Small differences are attributed to run statistics. (Runs performed using the separate isotopes are judged to produce the more accurate values.)

Results show that for both sources and concrete types, external dose rates at steady state are dominated by $\mathrm{Cu}-64$ and Mn-56 as was discussed in Section 2. In most cases the contribution from Mn-56 is up to several times higher and the combined contribution of $\mathrm{Cu}-64$ and Mn-56 is typically $\sim 98 \%$ of the total.

\section{CALCULATION AT LOCATIONS INSIDE THE FACILITY FOR STEADY-STATE OPERATION (AT SHUTDOWN), AND FOR 2 AND 10 DAYS AFTER SHUTDOWN}

For calculated values within the facility at specified locations horizontally or vertically from the components, a special version of the updated model was used in which the walls, floor, and ceiling were removed. Also, components for which the source was not being calculated were removed (i.e., ion exchanger was removed if filter source was used and vice versa). This assured that at locations at specified horizontal or vertical distances from a component (i.e., filter or ion chamber), there would be no influence from the surrounding structure, including the other component, on the results. In particular, since there is no scattering off the walls or otherwise, this approach removes the dependence on specific $(\mathrm{X}, \mathrm{Y})$ coordinates for a specified radial distance from a component outer surface. All calculations were performed using each isotope as a separate source and with the water and resin sources combined as in the isotopic calculations discussed in Section 3.

Dose rate values were obtained for three time stamps: shutdown, 2 days after shutdown, and 10 days after shutdown. The assumed purpose for examining different time periods was to obtain estimates of the waiting period following shutdown before inside components could be accessed (and to determine if additional shielding would be required) without exceeding the dose rate limit. At shutdown it is assumed that the source isotopes are no longer produced and dose rates begin to drop because of radioactive decay. A brief investigation into the decay modes of the isotopes considered showed that they all decay by simple means to stable isotopes. The assumption, which has been examined and is valid, is that no new isotopes and gamma lines are generated in the decay process following shutdown. Thus, the calculated dose rate from each isotope at times following shutdown can be determined by multiplying the results at shutdown by exponential decay factors based solely on the isotope's half-life. (Similarly, the activity of each isotope can be determined by multiplying the activity at shutdown by the same factor. This means that the conversion factor for each isotope for a specified source and location is independent of the decay time because the decay factor cancels out when the conversion factor is calculated.) Table 8 shows decay factors for each of the eight isotopes, where the decay factor is the fraction of the isotope remaining after a specified time following shutdown. Note that, because of their short half-lives, $\mathrm{Mn}-56$ and $\mathrm{Cu}-64$, the two largest source activity contributors at shutdown, decay rapidly, whereas Fe-55 and Co-60, minor contributors initially, scarcely change after 10 days because of their long half-lives. (As shown, the contribution from Fe-55 is actually negligible at all times.) Consequently, the isotopic compositions of the sources change considerably over a 10-day decay period. 
Tables 9 through 12 show results for tallies inside the structure at radial and axial distances from the outside surfaces of the ion exchanger and filter (for the ion exchanger, the radial values were those calculated at the vertical midplane of the resin region). Each table is devoted to a particular distance [5 cm, $30 \mathrm{~cm}, 50 \mathrm{~cm}, 100(75) \mathrm{cm}]$ and shows the results for the three time stamps. Dose rates contributed by each isotope are shown and the total dose rate summed over the isotopes is shown in the bottom row of each table for each distance. Dose rates that are within the $0.1 \mathrm{mSv} / \mathrm{h} \mathrm{limits}$ are highlighted as with green.

Table 13 is a convenient summary of the total dose rate values from the last rows of Tables 9 through 12.

From Tables 9 through 12, several conclusions can be drawn with regard to the isotopic contributions and are in general true for both components at all distances:

1) The dose rate contribution from $\mathrm{Fe}-55$ is negligible in all cases. Also, the contributions from Fe-59 and Cr-51 are small to negligible in all cases.

2) At shutdown the primary contributors are $\mathrm{Cu}-64$ and $\mathrm{Mn}-56$.

3) At 2 days after shutdown $\mathrm{Cu}-64$ is still the primary contributor, but the contribution from Mn-56 is negligible.

4) At 10 days after shutdown the primary contributors are Co-60, Co-58, and Mn-54 (largest to smallest) and the contribution from $\mathrm{Cu}-64$ is negligible.

From the summary in Table 13, the following conclusions can be drawn:

1) For vertical axis locations above the ion exchanger, dose rates are within the $0.1 \mathrm{mSv} / \mathrm{h}$ limit 2 days after shutdown for all distances.

2) For the horizontal radial locations from the ion exchanger, dose rates are within the $0.1 \mathrm{mSv} / \mathrm{h}$ limit 10 days after shutdown at distances of $50 \mathrm{~cm}$ and beyond.

3) For vertical and horizontal locations from the filter, dose rates are within the $0.1 \mathrm{mSv} / \mathrm{h}$ limit 10 days after shutdown at distances of $30 \mathrm{~cm}$ and beyond.

Table 14 provides an additional dose rate summarization, which shows the isotopic dose rate contributions at all the distances for both components. Values are shown only for shutdown. The attenuation of the contributions with distance can be clearly observed for all isotopes except Fe-55 for the ion exchanger, where the values remain nearly constant for the vertical axis and increase slightly with distance for the horizontal axis. The apparent increase with the distance of the dose produced by Fe-55 is most likely a numerical artifact and is of no concern given that the contribution of this isotope to the dose is many orders of magnitude below the contribution of any of the other isotopes.

Table 15 shows, in a format similar to Table 14, conversion factors for the isotope contributions, i.e., dose rates in Table 14 divided by the activity for each source isotope. Note that as explained earlier, the conversion factors are independent of isotope decay time.

\section{DOSE RATE CONTOUR PLOTS}

Figures 4 through 11 show dose rate contour plots extracted from combined MCNP mesh tally calculations for the combined sources. Contour plots are shown for 30-cm-thick walls/ceiling as follows:

1) Standard concrete walls/ceiling, at shutdown (Fig. 4 and 5)

2) Barytes concrete walls/ceiling, at shutdown (Fig. 6 and 7) 
3) Barytes concrete walls/ceiling, 2 days after shutdown (Fig. 8 and 9)

4) Barytes concrete walls/ceiling, 10 days after shutdown (Fig. 10 and 11)

These plots provide an overall perspective of how the dose rate varies in the geometry. For each pair of figures, the first figure shows values in the horizontal (XY) plane at the vertical centers of the filter, resin, and water, respectively, and the second figure shows values in the XZ plane for the filter and the ion exchanger at their $\mathrm{Y}$ axis centers. Dose rates are shown above $0.01 \mathrm{mSv} / \mathrm{h}(10 \%$ of the personnel limit $)$ for all images. For the shutdown, 2 days after shutdown, and 10 days after shutdown, the upper limits are 100,10 , and $1 \mathrm{mSv} / \mathrm{h}$, respectively. Several general observations can be made as follows:

1) For the standard concrete walls at shutdown, internal dose rates fall below the $0.1 \mathrm{mSv} / \mathrm{h}$ limit at $\sim 1200 \mathrm{~cm}$ from the short wall on the X-axis. Dose rates outside the walls are below the limit at most locations.

2) The plots at shutdown for the Barytes concrete walls/ceiling are essentially identical to those for the standard concrete, except that all values outside the walls/ceiling are below $0.1 \mathrm{mSv} / \mathrm{h}$.

3) The plots 2 days and 10 days after shutdown for the barytes concrete walls/ceiling show a dramatic decrease in dose rates. Note the extensive increase in "white" space in internal areas where dose rates are below the lower plot limit of $0.01 \mathrm{mSv} / \mathrm{h}$.

Values at exact locations are difficult to read from the plots but can be obtained from software used to read the MCNP mesh tallies and generate the plots.

\section{SUMMARY AND CONCLUSIONS}

For the simple model and the assumptions used (sources, materials, dimensions, etc.), the 30-cm-thick regular concrete walls/ceiling appear to provide sufficient shielding for the $0.1 \mathrm{mSv} / \mathrm{h}$ limit outside the facility at most locations, but was not sufficient at locations adjacent to the ceiling and long wall above and across from the filter. A standard concrete wall thickness of $15 \mathrm{~cm}$ was shown to be insufficient at all of the 10 specific cell-tally locations. At some distance along the $\mathrm{X}$ axis, not determined precisely in this analysis, the walls and ceiling thickness are of no concern for shielding the two components considered since the $0.1 \mathrm{mSv} / \mathrm{h}$ limit is met within the enclosure.

A barytes concrete wall of the same thickness $(15 \mathrm{~cm})$, however, is sufficient to reduce the dose rates to an acceptable level. The use of 30 -cm-thick barytes concrete in the walls/ceiling appears to be an overlyconservative design since the external dose rates are calculated to be at least an order-of-magnitude below the $0.1 \mathrm{mSv} / \mathrm{h}$ limit at almost all locations. However, it must again be mentioned that the model is simplified and, until a more detailed and sophisticated analysis is performed, it may be prudent to retain this as a conservative design.

Isotopic calculations show that during operation the external dose rate is dominated by contributions from Cu-64 and Mn-56 ( $\sim 98 \%$ of total) for standard concrete and barytes concrete walls/ceiling. At most locations, the contribution from Mn-56 is greater than that from $\mathrm{Cu}-64$ by up to several times.

Isotopic calculations were performed for the internal locations using a special model version in which all shielding and scattering components were removed (except air). Results for each isotope were also determined by modifying the results by the calculated amount of the isotope following decay for specified times after shutdown as follows: 0 days (at shutdown, no decay), 2 days, and 10 days. Results, which are discussed in detail in Section 4, are expected to provide some guidance as to when components could be approached without exceeding the $0.1 \mathrm{mSv} / \mathrm{h}$ limit. Again, at shutdown results are dominated by $\mathrm{Cu}-64$ and Mn-56. However, these isotopes decay quickly and the isotopic dominance changes over time 
(Tables 9 through 12). In general it is concluded that even after a 10 day waiting period, the ion exchanger cannot be approached within a radial distance of $<50 \mathrm{~cm}$, and the filter cannot be approached within an axial or radial distance of $<30 \mathrm{~cm}$. Consequently, the feasibility of using local or movable shielding may need to be addressed.

For all isotopic calculations, conversion factors (dose rate divided by activity) were calculated. This enables the estimation of dose rates for different mixtures of isotopes without having to perform additional MCNP calculations.

It is suggested that more detailed analyses be undertaken, which include additional components, piping, and wall penetrations, as well as the sensitivity of the results to the different material properties (densities, composition, dimensions). Dose rate evaluations for piping components and penetrations can lead to the necessity for local extra-shielding if the radiation field is to be maintained below the admissible limits.

Fluctuations in material properties can lead to underestimations of the dose by the reference model, which can be accounted for by additional safety factors.

Also an analysis of impact on dose distributions of the volumetric distribution (such as due to precipitation) of the nuclides in the tanks volumes should be evaluated. An inhomogeneous dose distribution can lead to a higher dose rate near regions with accumulation of radioactive nuclides. For example, if for any reason the radioactive nuclides accumulate preferentially near the wall, the dose rates outside the respective component would likely increase. Therefore, an investigation into the possibility that this can reasonably occur is recommended. 

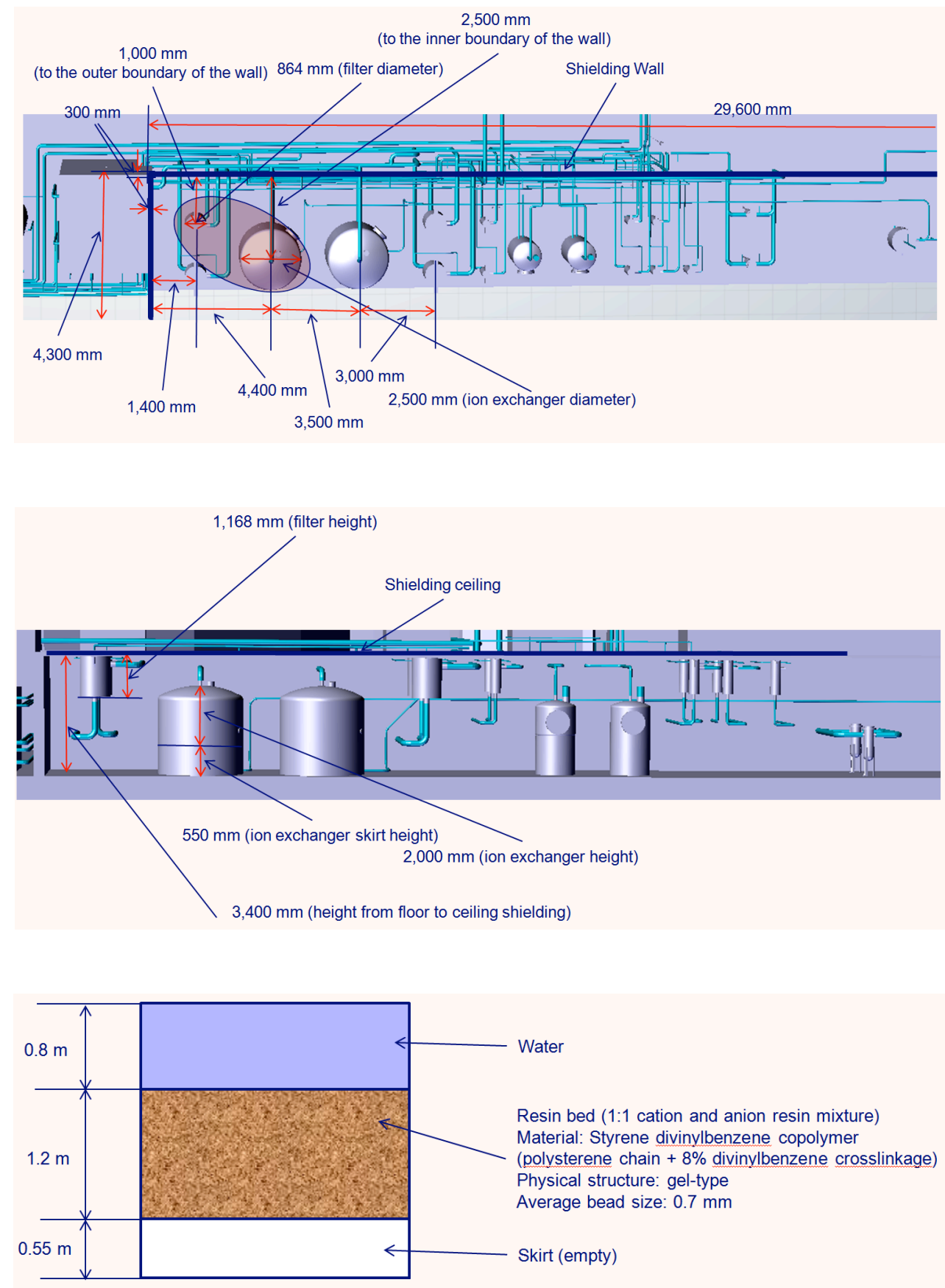

Fig. 1. Layout and dimensions of the shielding wall and equipment in the CVCS area. 


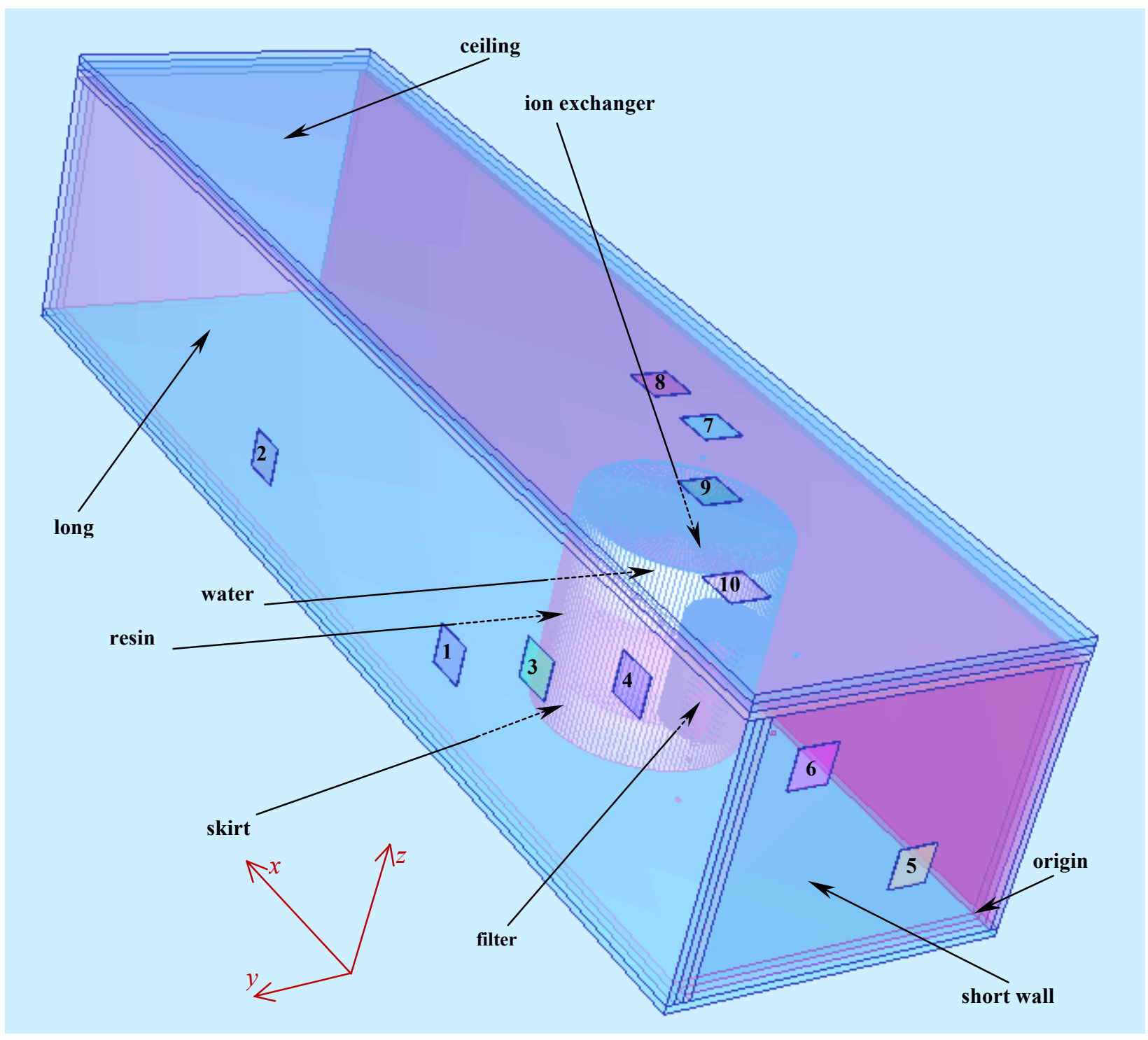

Fig. 2. View of MCNP model showing components and outside tally cells. 
Table 1. Locations of external dose rate tally cells

\begin{tabular}{|c|c|c|c|}
\hline \multirow[t]{2}{*}{ No. } & \multicolumn{2}{|r|}{ Description of external tally cell location } & \multirow{2}{*}{$\begin{array}{c}X, Y, Z(\mathbf{c m}, \mathbf{c m}, \mathbf{c m}) \\
\text { location of tally cell } \\
\text { center }\end{array}$} \\
\hline & $\begin{array}{l}\text { Wall/ } \\
\text { Ceiling }\end{array}$ & Other axes location & \\
\hline 1 & Long & $(\mathrm{X}, \mathrm{Z})$ coordinates at center of resin. & $440,431,115.55$ \\
\hline 2 & Long & Same as 1 , but X-coordinate increased $440 \mathrm{~cm}$. & $880,431,115.55$ \\
\hline 3 & Long & $(\mathrm{X}, \mathrm{Z})$ coordinates halfway between resin and filter centers. & $290,431,193.58$ \\
\hline 4 & Long & $(\mathrm{X}, \mathrm{Z})$ coordinates at center of filter. & $140,431,271.6$ \\
\hline 5 & Short & $\begin{array}{l}\mathrm{X} \text {-coordinate outside short (Y-axis) wall. }(\mathrm{Y}, \mathrm{Z}) \text { coordinates } \\
\text { at center of resin. }\end{array}$ & $-31,150,115.55$ \\
\hline 6 & Short & $\mathrm{X}$-coordinate same as $5 .(\mathrm{Y}, \mathrm{Z})$ coordinates at center of filter. & $-31,330,271.6$ \\
\hline 7 & Ceiling & $(\mathrm{X}, \mathrm{Y})$ coordinates at center of ion exchanger. & $440,150,371$ \\
\hline 8 & Ceiling & Same as 7, but $X$-coordinate increased $100 \mathrm{~cm}$. & $540,150,371$ \\
\hline 9 & Ceiling & $\begin{array}{l}(\mathrm{X}, \mathrm{Y}) \text { coordinates halfway between ion exchanger and filter } \\
\text { centers. }\end{array}$ & $315,240,371$ \\
\hline 10 & Ceiling & $(\mathrm{X}, \mathrm{Y})$ coordinates at center of filter. & $140,330,371$ \\
\hline
\end{tabular}

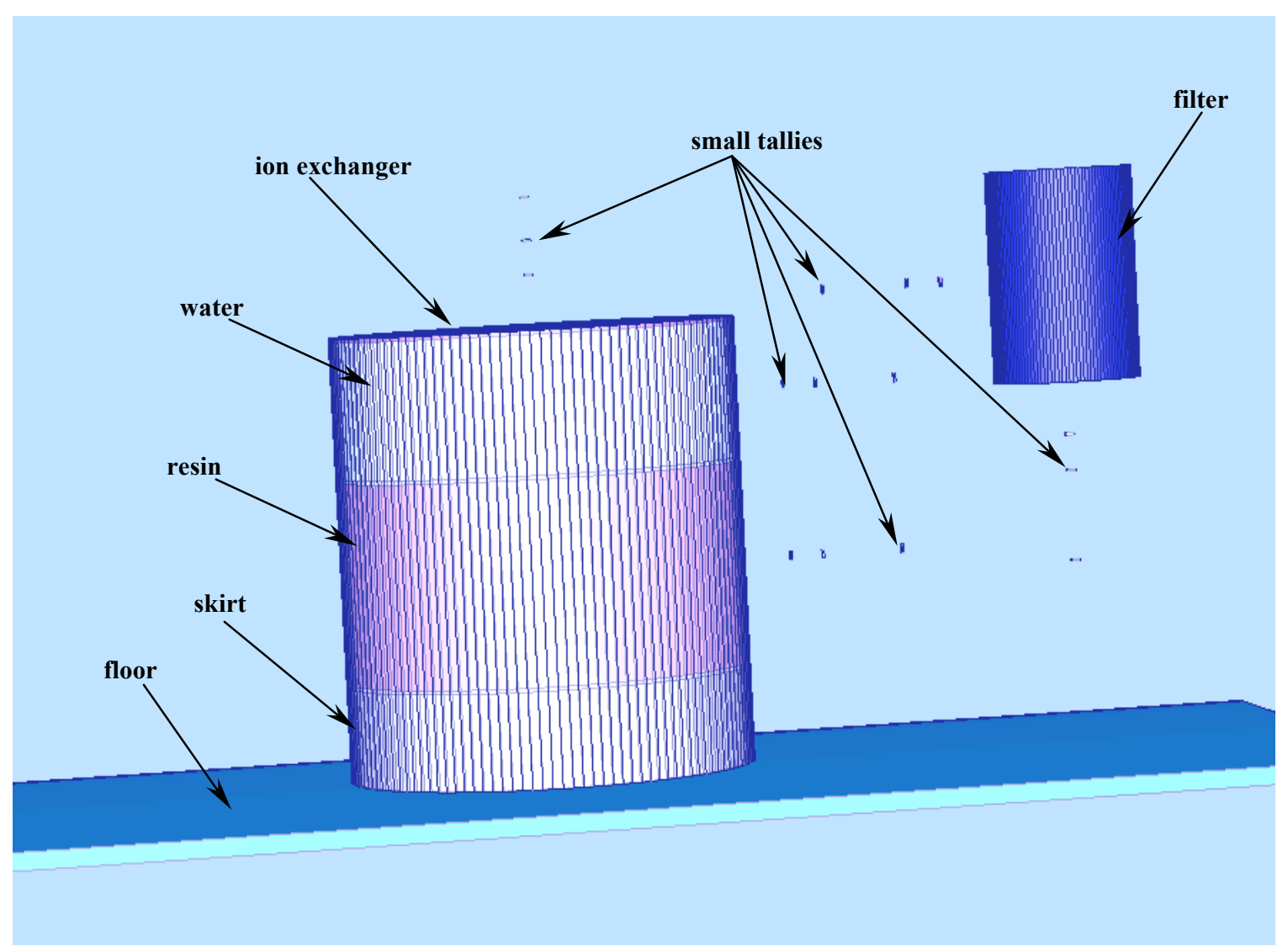

Fig. 3. Detailed inside view showing components and small internal tally cells. 
Table 2. Calculation of photon sources (steady state)

\begin{tabular}{|c|c|c|c|c|c|c|c|c|}
\hline \multirow[b]{2}{*}{ Isotope } & \multirow[b]{2}{*}{ E (keV) } & \multirow[b]{2}{*}{$\%$-fract. } & \multicolumn{3}{|c|}{ Activity (GBq) } & \multicolumn{3}{|c|}{ Source $\mathrm{x}$ fract. (photons/s) } \\
\hline & & & filter & $\begin{array}{c}\text { ion-exc. } \\
\text { resin }\end{array}$ & $\begin{array}{c}\text { ion-exc. } \\
\text { water }\end{array}$ & filter & $\begin{array}{c}\text { ion-exc. } \\
\text { resin }\end{array}$ & $\begin{array}{c}\text { ion-exc. } \\
\text { water }\end{array}$ \\
\hline \multirow[t]{8}{*}{ Fe59 } & 142.651 & 1.02 & 0.05 & 0.54 & 0.0009 & $5.100 \mathrm{E}+05$ & $5.508 \mathrm{E}+06$ & $9.180 \mathrm{E}+03$ \\
\hline & 189 & $9.00 \mathrm{E}-04$ & & & & $4.500 \mathrm{E}+02$ & $4.860 \mathrm{E}+03$ & $8.100 \mathrm{E}+00$ \\
\hline & 192.343 & 3.08 & & & & $1.540 \mathrm{E}+06$ & $1.663 \mathrm{E}+07$ & $2.772 \mathrm{E}+04$ \\
\hline & 334.8 & 0.27 & & & & $1.350 \mathrm{E}+05$ & $1.458 \mathrm{E}+06$ & $2.430 \mathrm{E}+03$ \\
\hline & 382 & 0.018 & & & & $9.000 \mathrm{E}+03$ & $9.720 \mathrm{E}+04$ & $1.620 \mathrm{E}+02$ \\
\hline & 1099.245 & 56.5 & & & & $2.825 \mathrm{E}+07$ & $3.051 \mathrm{E}+08$ & $5.085 \mathrm{E}+05$ \\
\hline & 1291.59 & 43.2 & & & & $2.160 \mathrm{E}+07$ & $2.333 \mathrm{E}+08$ & $3.888 \mathrm{E}+05$ \\
\hline & 1481.7 & 0.059 & & & & $2.950 \mathrm{E}+04$ & $3.186 \mathrm{E}+05$ & $5.310 \mathrm{E}+02$ \\
\hline \multirow[t]{2}{*}{ Mn54 } & 511 & $6.00 \mathrm{E}-07$ & 0.34 & 3.43 & 0.0021 & $2.040 \mathrm{E}+00$ & $2.058 \mathrm{E}+01$ & $1.260 \mathrm{E}-02$ \\
\hline & 834.848 & 99.976 & & & & $3.399 \mathrm{E}+08$ & $3.429 \mathrm{E}+09$ & $2.099 \mathrm{E}+06$ \\
\hline $\mathrm{Fe} 55$ & 126 & $1.28 \mathrm{E}-07$ & 0.16 & 2.96 & 0.0015 & $2.048 \mathrm{E}-01$ & $3.789 \mathrm{E}+00$ & $1.920 \mathrm{E}-03$ \\
\hline \multirow[t]{4}{*}{ Co58 } & 511 & 29.8 & 0.52 & 5.18 & 0.0059 & $1.550 \mathrm{E}+08$ & $1.544 \mathrm{E}+09$ & $1.758 \mathrm{E}+06$ \\
\hline & 810.7593 & 99.45 & & & & $5.171 \mathrm{E}+08$ & $5.152 \mathrm{E}+09$ & $5.868 \mathrm{E}+06$ \\
\hline & 863.951 & 0.686 & & & & $3.567 \mathrm{E}+06$ & $3.553 \mathrm{E}+07$ & $4.047 \mathrm{E}+04$ \\
\hline & 1674.725 & 0.517 & & & & $2.688 \mathrm{E}+06$ & $2.678 \mathrm{E}+07$ & $3.050 \mathrm{E}+04$ \\
\hline \multirow[t]{6}{*}{ Co60 } & 347.14 & 0.0075 & 0.28 & 2.79 & 0.0014 & $2.100 \mathrm{E}+04$ & $2.093 \mathrm{E}+05$ & $1.050 \mathrm{E}+02$ \\
\hline & 826.1 & 0.0076 & & & & $2.128 \mathrm{E}+04$ & $2.120 \mathrm{E}+05$ & $1.064 \mathrm{E}+02$ \\
\hline & 1173.228 & 99.85 & & & & $2.796 \mathrm{E}+08$ & $2.786 \mathrm{E}+09$ & $1.398 \mathrm{E}+06$ \\
\hline & 1332.492 & 99.9826 & & & & $2.800 \mathrm{E}+08$ & $2.790 \mathrm{E}+09$ & $1.400 \mathrm{E}+06$ \\
\hline & 2158.57 & 0.0012 & & & & $3.360 \mathrm{E}+03$ & $3.348 \mathrm{E}+04$ & $1.680 \mathrm{E}+01$ \\
\hline & 2505.692 & $2.00 \mathrm{E}-06$ & & & & $5.600 \mathrm{E}+00$ & $5.580 \mathrm{E}+01$ & $2.800 \mathrm{E}-02$ \\
\hline Cr51 & 320.0824 & 9.91 & 1.78 & 17.84 & 0.0043 & $1.764 \mathrm{E}+08$ & $1.768 \mathrm{E}+09$ & $4.261 \mathrm{E}+05$ \\
\hline \multirow[t]{10}{*}{ Mn56 } & 846.754 & 98.9 & 16.94 & 169.37 & 43.72 & $1.675 \mathrm{E}+10$ & $1.675 \mathrm{E}+11$ & $4.324 \mathrm{E}+10$ \\
\hline & 1037.818 & 0.04 & & & & $6.776 \mathrm{E}+06$ & $6.775 \mathrm{E}+07$ & $1.749 \mathrm{E}+07$ \\
\hline & 1238.255 & 0.099 & & & & $1.677 \mathrm{E}+07$ & $1.677 \mathrm{E}+08$ & $4.328 \mathrm{E}+07$ \\
\hline & 1810.72 & 27.2 & & & & $4.608 \mathrm{E}+09$ & $4.607 \mathrm{E}+10$ & $1.189 \mathrm{E}+10$ \\
\hline & 2113.05 & 14.3 & & & & $2.422 \mathrm{E}+09$ & $2.422 \mathrm{E}+10$ & $6.252 \mathrm{E}+09$ \\
\hline & 2522.88 & 0.99 & & & & $1.677 \mathrm{E}+08$ & $1.677 \mathrm{E}+09$ & $4.328 \mathrm{E}+08$ \\
\hline & 2598.4 & 0.0188 & & & & $3.185 \mathrm{E}+06$ & $3.184 \mathrm{E}+07$ & $8.219 \mathrm{E}+06$ \\
\hline & 2657.45 & 0.653 & & & & $1.106 \mathrm{E}+08$ & $1.106 \mathrm{E}+09$ & $2.855 \mathrm{E}+08$ \\
\hline & 2959.77 & 0.306 & & & & $5.184 \mathrm{E}+07$ & $5.183 \mathrm{E}+08$ & $1.338 \mathrm{E}+08$ \\
\hline & 3369.6 & 0.168 & & & & $2.846 \mathrm{E}+07$ & $2.845 \mathrm{E}+08$ & $7.345 \mathrm{E}+07$ \\
\hline \multirow[t]{2}{*}{$\mathrm{Cu} 64$} & 511 & 35.2 & 708.06 & 7080.58 & 587.74 & $2.492 \mathrm{E}+11$ & $2.492 \mathrm{E}+12$ & $2.069 \mathrm{E}+11$ \\
\hline & 1345.77 & 0.475 & & & & $3.363 \mathrm{E}+09$ & $3.363 \mathrm{E}+10$ & $2.792 \mathrm{E}+09$ \\
\hline \multicolumn{3}{|c|}{ TOTAL ACTIVITY } & 728.13 & 7282.69 & 631.48 & & & \\
\hline \multicolumn{3}{|c|}{ TOTAL COMBINED ACTIVITY } & & & 8642.3 & & & \\
\hline \multicolumn{6}{|c|}{ TOTAL PHOTON SOURCE (photons/s) } & $2.786 \mathrm{E}+11$ & $2.786 \mathrm{E}+12$ & $2.721 \mathrm{E}+11$ \\
\hline \multicolumn{6}{|c|}{ TOTAL PHOTON SOURCE/ACTIVITY (photons/s)/GBq) } & $3.826 \mathrm{E}+08$ & $3.825 \mathrm{E}+08$ & $4.308 \mathrm{E}+08$ \\
\hline
\end{tabular}


Table 3. External dose rates for separate and combined sources

30-cm-thick wall/ceiling, standard concrete

\begin{tabular}{|c|c|c|c|c|c|}
\hline \multirow{3}{*}{ Cell No. } & \multirow{3}{*}{$\begin{array}{c}\text { Wall/ } \\
\text { Ceiling }\end{array}$} & \multicolumn{4}{|c|}{ Dose Rate (mSv/h) } \\
\hline & & \multicolumn{3}{|c|}{ Source } & \multirow[b]{2}{*}{ TOTAL } \\
\hline & & Filter & Resin & Water & \\
\hline 1 & Long & $1.88 \mathrm{E}-04$ & $5.49 \mathrm{E}-02$ & $4.80 \mathrm{E}-03$ & $5.99 \mathrm{E}-02$ \\
\hline 2 & Long & $1.40 \mathrm{E}-05$ & $1.99 \mathrm{E}-03$ & $3.39 \mathrm{E}-04$ & $2.34 \mathrm{E}-03$ \\
\hline 3 & Long & $3.23 \mathrm{E}-03$ & $2.00 \mathrm{E}-02$ & $5.90 \mathrm{E}-03$ & $2.91 \mathrm{E}-02$ \\
\hline 4 & Long & $9.73 \mathrm{E}-02$ & $3.07 \mathrm{E}-03$ & $1.32 \mathrm{E}-03$ & $1.02 \mathrm{E}-01$ \\
\hline 5 & Short & $2.12 \mathrm{E}-03$ & $1.81 \mathrm{E}-02$ & $2.79 \mathrm{E}-03$ & $2.30 \mathrm{E}-02$ \\
\hline 6 & Short & 4.22E-02 & $6.20 \mathrm{E}-03$ & $1.74 \mathrm{E}-03$ & $5.01 \mathrm{E}-02$ \\
\hline 7 & Ceiling & $2.02 \mathrm{E}-04$ & $7.90 \mathrm{E}-04$ & $4.33 \mathrm{E}-02$ & $4.43 \mathrm{E}-02$ \\
\hline 8 & Ceiling & 8.38E-05 & $5.45 \mathrm{E}-04$ & $2.73 \mathrm{E}-02$ & $2.79 \mathrm{E}-02$ \\
\hline 9 & Ceiling & $2.05 \mathrm{E}-03$ & $5.81 \mathrm{E}-04$ & $1.14 \mathrm{E}-02$ & $1.41 \mathrm{E}-02$ \\
\hline 10 & Ceiling & $1.29 \mathrm{E}-01$ & $1.34 \mathrm{E}-03$ & $4.02 \mathrm{E}-04$ & $1.31 \mathrm{E}-01$ \\
\hline
\end{tabular}

15-cm-thick wall/ceiling, standard concrete

\begin{tabular}{|c|c|c|c|c|c|}
\hline \multirow{3}{*}{ Cell No. } & \multirow{3}{*}{$\begin{array}{c}\text { Wall/ } \\
\text { Ceiling }\end{array}$} & \multicolumn{4}{|c|}{ Dose Rate (mSv/h) } \\
\hline & & \multicolumn{3}{|c|}{ Source } & \multirow[b]{2}{*}{ TOTAL } \\
\hline & & Filter & Resin & Water & \\
\hline 1 & Long & 4.13E-03 & $3.77 \mathrm{E}-01$ & $3.02 \mathrm{E}-02$ & 4.12E-01 \\
\hline 2 & Long & $3.18 \mathrm{E}-04$ & $2.62 \mathrm{E}-02$ & $3.80 \mathrm{E}-03$ & $3.03 \mathrm{E}-02$ \\
\hline 3 & Long & 4.73E-02 & $1.64 \mathrm{E}-01$ & $3.70 \mathrm{E}-02$ & $2.48 \mathrm{E}-01$ \\
\hline 4 & Long & $7.59 \mathrm{E}-01$ & $3.50 \mathrm{E}-02$ & $1.11 \mathrm{E}-02$ & $8.05 \mathrm{E}-01$ \\
\hline 5 & Short & $2.82 \mathrm{E}-02$ & $1.22 \mathrm{E}-01$ & $1.60 \mathrm{E}-02$ & $1.66 \mathrm{E}-01$ \\
\hline 6 & Short & $3.13 \mathrm{E}-01$ & 4.99E-02 & $1.07 \mathrm{E}-02$ & 3.74E-01 \\
\hline 7 & Ceiling & 4.11E-03 & $3.84 \mathrm{E}-03$ & $2.68 \mathrm{E}-01$ & $2.76 \mathrm{E}-01$ \\
\hline 8 & Ceiling & $1.81 \mathrm{E}-03$ & $3.71 \mathrm{E}-03$ & $1.71 \mathrm{E}-01$ & $1.77 \mathrm{E}-01$ \\
\hline 9 & Ceiling & $3.05 \mathrm{E}-02$ & $6.05 \mathrm{E}-03$ & 7.94E-02 & $1.16 \mathrm{E}-01$ \\
\hline 10 & Ceiling & $1.05 \mathrm{E}+00$ & $1.43 \mathrm{E}-02$ & $5.08 \mathrm{E}-03$ & $1.07 \mathrm{E}+00$ \\
\hline
\end{tabular}

30-cm-thick wall/ceiling, Barytes concrete

\begin{tabular}{|c|c|c|c|c|c|}
\hline \multirow{3}{*}{ Cell No. } & \multirow{3}{*}{$\begin{array}{c}\text { Wall/ } \\
\text { Ceiling }\end{array}$} & \multicolumn{4}{|c|}{ Dose Rate (mSv/h) } \\
\hline & & \multicolumn{3}{|c|}{ Source } & \multirow[b]{2}{*}{ TOTAL } \\
\hline & & Filter & Resin & Water & \\
\hline 1 & Long & $7.96 \mathrm{E}-06$ & $9.60 \mathrm{E}-03$ & $9.76 \mathrm{E}-04$ & $1.06 \mathrm{E}-02$ \\
\hline 2 & Long & $3.27 \mathrm{E}-07$ & $1.97 \mathrm{E}-04$ & $4.11 \mathrm{E}-05$ & $2.38 \mathrm{E}-04$ \\
\hline 3 & Long & $3.01 \mathrm{E}-04$ & $3.18 \mathrm{E}-03$ & $1.19 \mathrm{E}-03$ & $4.67 \mathrm{E}-03$ \\
\hline 4 & Long & $1.45 \mathrm{E}-02$ & $3.89 \mathrm{E}-04$ & $2.13 \mathrm{E}-04$ & $1.52 \mathrm{E}-02$ \\
\hline 5 & Short & $2.13 \mathrm{E}-04$ & $3.19 \mathrm{E}-03$ & $6.13 \mathrm{E}-04$ & $4.02 \mathrm{E}-03$ \\
\hline 6 & Short & $6.60 \mathrm{E}-03$ & $1.02 \mathrm{E}-03$ & $3.71 \mathrm{E}-04$ & $7.98 \mathrm{E}-03$ \\
\hline 7 & Ceiling & $9.30 \mathrm{E}-06$ & $1.97 \mathrm{E}-04$ & $8.75 \mathrm{E}-03$ & $8.96 \mathrm{E}-03$ \\
\hline 8 & Ceiling & $3.07 \mathrm{E}-06$ & $1.25 \mathrm{E}-04$ & $5.48 \mathrm{E}-03$ & $5.61 \mathrm{E}-03$ \\
\hline 9 & Ceiling & $1.81 \mathrm{E}-04$ & $9.24 \mathrm{E}-05$ & $2.16 \mathrm{E}-03$ & $2.43 \mathrm{E}-03$ \\
\hline 10 & Ceiling & $1.83 \mathrm{E}-02$ & $2.07 \mathrm{E}-04$ & $4.69 \mathrm{E}-05$ & $1.86 \mathrm{E}-02$ \\
\hline
\end{tabular}


Table 4. Dose Rates $(\mathrm{mSv} / \mathrm{h})$ for individual isotopes at external locations (30-cm thick wall, standard concrete)

Ion Exchanger

\begin{tabular}{|l|c|c|c|c|c|c|c|c|c|c|}
\hline \multirow{2}{*}{ Isotope } & \multicolumn{10}{|c|}{ Location number (numbering as in Fig. 1) } \\
\cline { 2 - 12 } & $\mathbf{1}$ & $\mathbf{2}$ & $\mathbf{3}$ & $\mathbf{4}$ & $\mathbf{5}$ & $\mathbf{6}$ & $\mathbf{7}$ & $\mathbf{8}$ & $\mathbf{9}$ & $\mathbf{1 0}$ \\
\hline Fe-59 & $5.14 \mathrm{E}-05$ & $1.77 \mathrm{E}-06$ & $1.86 \mathrm{E}-05$ & $2.78 \mathrm{E}-06$ & $1.68 \mathrm{E}-05$ & $5.84 \mathrm{E}-06$ & $7.79 \mathrm{E}-07$ & $4.98 \mathrm{E}-07$ & $4.92 \mathrm{E}-07$ & $1.18 \mathrm{E}-06$ \\
\hline $\mathrm{Mn}-54$ & $1.17 \mathrm{E}-04$ & $3.71 \mathrm{E}-06$ & $3.99 \mathrm{E}-05$ & $5.45 \mathrm{E}-06$ & $3.83 \mathrm{E}-05$ & $1.23 \mathrm{E}-05$ & $6.46 \mathrm{E}-07$ & $4.82 \mathrm{E}-07$ & $8.98 \mathrm{E}-07$ & $1.93 \mathrm{E}-06$ \\
\hline Fe-55 & $4.73 \mathrm{E}-18$ & $5.11 \mathrm{E}-20$ & $7.30 \mathrm{E}-18$ & $3.76 \mathrm{E}-16$ & $3.46 \mathrm{E}-18$ & $1.80 \mathrm{E}-16$ & $9.70 \mathrm{E}-19$ & $2.18 \mathrm{E}-19$ & $3.86 \mathrm{E}-18$ & $5.36 \mathrm{E}-16$ \\
\hline $\mathrm{Co}-58$ & $1.80 \mathrm{E}-04$ & $5.75 \mathrm{E}-06$ & $6.09 \mathrm{E}-05$ & $8.18 \mathrm{E}-06$ & $5.87 \mathrm{E}-05$ & $1.88 \mathrm{E}-05$ & $1.44 \mathrm{E}-06$ & $1.01 \mathrm{E}-06$ & $1.48 \mathrm{E}-06$ & $2.94 \mathrm{E}-06$ \\
\hline $\mathrm{Co}-60$ & $6.20 \mathrm{E}-04$ & $2.18 \mathrm{E}-05$ & $2.26 \mathrm{E}-04$ & $3.40 \mathrm{E}-05$ & $2.02 \mathrm{E}-04$ & $7.08 \mathrm{E}-05$ & $6.80 \mathrm{E}-06$ & $4.31 \mathrm{E}-06$ & $5.35 \mathrm{E}-06$ & $1.47 \mathrm{E}-05$ \\
\hline $\mathrm{Cr}-51$ & $2.06 \mathrm{E}-06$ & $6.06 \mathrm{E}-08$ & $6.19 \mathrm{E}-07$ & $7.68 \mathrm{E}-08$ & $6.68 \mathrm{E}-07$ & $1.91 \mathrm{E}-07$ & $2.84 \mathrm{E}-09$ & $5.06 \mathrm{E}-09$ & $1.76 \mathrm{E}-08$ & $1.56 \mathrm{E}-08$ \\
\hline $\mathrm{Mn}-56$ & $3.60 \mathrm{E}-02$ & $1.56 \mathrm{E}-03$ & $1.72 \mathrm{E}-02$ & $3.19 \mathrm{E}-03$ & $1.27 \mathrm{E}-02$ & $5.27 \mathrm{E}-03$ & $3.64 \mathrm{E}-02$ & $2.30 \mathrm{E}-02$ & $1.01 \mathrm{E}-02$ & $1.39 \mathrm{E}-03$ \\
\hline $\mathrm{Cu}-64$ & $2.29 \mathrm{E}-02$ & $7.37 \mathrm{E}-04$ & $8.17 \mathrm{E}-03$ & $1.15 \mathrm{E}-03$ & $7.72 \mathrm{E}-03$ & $2.53 \mathrm{E}-03$ & $7.68 \mathrm{E}-03$ & $4.82 \mathrm{E}-03$ & $1.90 \mathrm{E}-03$ & $3.55 \mathrm{E}-04$ \\
\hline TOTAL & $5.99 \mathrm{E}-02$ & $2.33 \mathrm{E}-03$ & $2.58 \mathrm{E}-02$ & $4.39 \mathrm{E}-03$ & $2.07 \mathrm{E}-02$ & $7.91 \mathrm{E}-03$ & $4.41 \mathrm{E}-02$ & $2.78 \mathrm{E}-02$ & $1.20 \mathrm{E}-02$ & $1.76 \mathrm{E}-03$ \\
\hline
\end{tabular}

Filter

\begin{tabular}{|l|c|c|c|c|c|c|c|c|c|c|}
\hline \multirow{2}{*}{ Isotope } & \multicolumn{9}{|c|}{ Location number (numbering as in Fig. 1) } \\
\cline { 2 - 12 } & $\mathbf{1}$ & $\mathbf{2}$ & $\mathbf{3}$ & $\mathbf{4}$ & $\mathbf{5}$ & $\mathbf{6}$ & $\mathbf{7}$ & $\mathbf{8}$ & $\mathbf{9}$ & $\mathbf{1 0}$ \\
\hline Fe-59 & $1.39 \mathrm{E}-07$ & $9.79 \mathrm{E}-09$ & $2.59 \mathrm{E}-06$ & $8.00 \mathrm{E}-05$ & $1.70 \mathrm{E}-06$ & $3.45 \mathrm{E}-05$ & $1.50 \mathrm{E}-07$ & $6.23 \mathrm{E}-08$ & $1.62 \mathrm{E}-06$ & $1.05 \mathrm{E}-04$ \\
\hline $\mathrm{Mn}-54$ & $4.01 \mathrm{E}-07$ & $2.98 \mathrm{E}-08$ & $6.17 \mathrm{E}-06$ & $2.15 \mathrm{E}-04$ & $4.04 \mathrm{E}-06$ & $9.41 \mathrm{E}-05$ & $4.17 \mathrm{E}-07$ & $1.85 \mathrm{E}-07$ & $4.01 \mathrm{E}-06$ & $2.87 \mathrm{E}-04$ \\
\hline Fe-55 & $8.26 \mathrm{E}-20$ & $4.58 \mathrm{E}-21$ & $7.43 \mathrm{E}-19$ & $4.06 \mathrm{E}-17$ & $3.83 \mathrm{E}-19$ & $1.94 \mathrm{E}-17$ & $8.45 \mathrm{E}-20$ & $2.67 \mathrm{E}-20$ & $4.28 \mathrm{E}-19$ & $5.86 \mathrm{E}-17$ \\
\hline Co-58 & $6.46 \mathrm{E}-07$ & $4.91 \mathrm{E}-08$ & $9.62 \mathrm{E}-06$ & $3.37 \mathrm{E}-04$ & $6.31 \mathrm{E}-06$ & $1.48 \mathrm{E}-04$ & $6.71 \mathrm{E}-07$ & $2.93 \mathrm{E}-07$ & $6.31 \mathrm{E}-06$ & $4.52 \mathrm{E}-04$ \\
\hline Co-60 & $1.80 \mathrm{E}-06$ & $1.24 \mathrm{E}-07$ & $3.43 \mathrm{E}-05$ & $1.03 \mathrm{E}-03$ & $2.24 \mathrm{E}-05$ & $4.45 \mathrm{E}-04$ & $1.94 \mathrm{E}-06$ & $7.97 \mathrm{E}-07$ & $2.13 \mathrm{E}-05$ & $1.36 \mathrm{E}-03$ \\
\hline $\mathrm{Cr}-51$ & $1.30 \mathrm{E}-08$ & $1.23 \mathrm{E}-09$ & $1.40 \mathrm{E}-07$ & $5.60 \mathrm{E}-06$ & $8.86 \mathrm{E}-08$ & $2.53 \mathrm{E}-06$ & $1.29 \mathrm{E}-08$ & $5.82 \mathrm{E}-09$ & $9.60 \mathrm{E}-08$ & $7.77 \mathrm{E}-06$ \\
\hline $\mathrm{Mn}-56$ & $9.21 \mathrm{E}-05$ & $5.22 \mathrm{E}-06$ & $1.95 \mathrm{E}-03$ & $5.04 \mathrm{E}-02$ & $1.26 \mathrm{E}-03$ & $2.14 \mathrm{E}-02$ & $1.01 \mathrm{E}-04$ & $3.94 \mathrm{E}-05$ & $1.18 \mathrm{E}-03$ & $6.54 \mathrm{E}-02$ \\
\hline $\mathrm{Cu}-64$ & $9.37 \mathrm{E}-05$ & $8.64 \mathrm{E}-06$ & $1.25 \mathrm{E}-03$ & $4.53 \mathrm{E}-02$ & $8.15 \mathrm{E}-04$ & $2.01 \mathrm{E}-02$ & $9.62 \mathrm{E}-05$ & $4.48 \mathrm{E}-05$ & $8.26 \mathrm{E}-04$ & $6.15 \mathrm{E}-02$ \\
\hline TOTAL & $1.89 \mathrm{E}-04$ & $1.41 \mathrm{E}-05$ & $3.25 \mathrm{E}-03$ & $9.74 \mathrm{E}-02$ & $2.11 \mathrm{E}-03$ & $4.22 \mathrm{E}-02$ & $2.00 \mathrm{E}-04$ & $8.56 \mathrm{E}-05$ & $2.04 \mathrm{E}-03$ & $1.29 \mathrm{E}-01$ \\
\hline
\end{tabular}

Ion Exchanger + Filter

\begin{tabular}{|l|c|c|c|c|c|c|c|c|c|c|}
\hline \multirow{2}{*}{ Isotope } & \multicolumn{10}{|c|}{ Location number (numbering as in Fig. 1) } \\
\cline { 2 - 11 } & $\mathbf{1}$ & $\mathbf{2}$ & $\mathbf{3}$ & $\mathbf{4}$ & $\mathbf{5}$ & $\mathbf{6}$ & $\mathbf{7}$ & $\mathbf{8}$ & $\mathbf{9}$ & $\mathbf{1 0}$ \\
\hline Fe-59 & $5.15 \mathrm{E}-05$ & $1.78 \mathrm{E}-06$ & $2.12 \mathrm{E}-05$ & $8.27 \mathrm{E}-05$ & $1.85 \mathrm{E}-05$ & $4.04 \mathrm{E}-05$ & $9.29 \mathrm{E}-07$ & $5.60 \mathrm{E}-07$ & $2.11 \mathrm{E}-06$ & $1.06 \mathrm{E}-04$ \\
\hline $\mathrm{Mn}-54$ & $1.18 \mathrm{E}-04$ & $3.74 \mathrm{E}-06$ & $4.60 \mathrm{E}-05$ & $2.20 \mathrm{E}-04$ & $4.24 \mathrm{E}-05$ & $1.06 \mathrm{E}-04$ & $1.06 \mathrm{E}-06$ & $6.67 \mathrm{E}-07$ & $4.91 \mathrm{E}-06$ & $2.89 \mathrm{E}-04$ \\
\hline Fe-55 & $4.81 \mathrm{E}-18$ & $5.57 \mathrm{E}-20$ & $8.04 \mathrm{E}-18$ & $4.16 \mathrm{E}-16$ & $3.84 \mathrm{E}-18$ & $1.99 \mathrm{E}-16$ & $1.05 \mathrm{E}-18$ & $2.45 \mathrm{E}-19$ & $4.29 \mathrm{E}-18$ & $5.94 \mathrm{E}-16$ \\
\hline Co-58 & $1.80 \mathrm{E}-04$ & $5.80 \mathrm{E}-06$ & $7.05 \mathrm{E}-05$ & $3.45 \mathrm{E}-04$ & $6.50 \mathrm{E}-05$ & $1.67 \mathrm{E}-04$ & $2.11 \mathrm{E}-06$ & $1.30 \mathrm{E}-06$ & $7.78 \mathrm{E}-06$ & $4.55 \mathrm{E}-04$ \\
\hline Co-60 & $6.22 \mathrm{E}-04$ & $2.19 \mathrm{E}-05$ & $2.61 \mathrm{E}-04$ & $1.07 \mathrm{E}-03$ & $2.25 \mathrm{E}-04$ & $5.16 \mathrm{E}-04$ & $8.74 \mathrm{E}-06$ & $5.11 \mathrm{E}-06$ & $2.67 \mathrm{E}-05$ & $1.37 \mathrm{E}-03$ \\
\hline Cr-51 & $2.07 \mathrm{E}-06$ & $6.18 \mathrm{E}-08$ & $7.59 \mathrm{E}-07$ & $5.67 \mathrm{E}-06$ & $7.57 \mathrm{E}-07$ & $2.72 \mathrm{E}-06$ & $1.57 \mathrm{E}-08$ & $1.09 \mathrm{E}-08$ & $1.14 \mathrm{E}-07$ & $7.79 \mathrm{E}-06$ \\
\hline Mn-56 & $3.61 \mathrm{E}-02$ & $1.57 \mathrm{E}-03$ & $1.92 \mathrm{E}-02$ & $5.36 \mathrm{E}-02$ & $1.39 \mathrm{E}-02$ & $2.67 \mathrm{E}-02$ & $3.65 \mathrm{E}-02$ & $2.30 \mathrm{E}-02$ & $1.13 \mathrm{E}-02$ & $6.68 \mathrm{E}-02$ \\
\hline Cu-64 & $2.30 \mathrm{E}-02$ & $7.46 \mathrm{E}-04$ & $9.42 \mathrm{E}-03$ & $4.65 \mathrm{E}-02$ & $8.53 \mathrm{E}-03$ & $2.27 \mathrm{E}-02$ & $7.77 \mathrm{E}-03$ & $4.87 \mathrm{E}-03$ & $2.72 \mathrm{E}-03$ & $6.19 \mathrm{E}-02$ \\
\hline TOTAL & $6.01 \mathrm{E}-02$ & $2.34 \mathrm{E}-03$ & $2.90 \mathrm{E}-02$ & $1.02 \mathrm{E}-01$ & $2.28 \mathrm{E}-02$ & $5.02 \mathrm{E}-02$ & $4.43 \mathrm{E}-02$ & $2.79 \mathrm{E}-02$ & $1.40 \mathrm{E}-02$ & $1.31 \mathrm{E}-01$ \\
\hline
\end{tabular}


Table 5. Conversion factors ( $\mathrm{mSv} / \mathrm{h}-\mathrm{per}-\mathrm{GBq})$ for individual isotopes at external locations (30-cm thick wall, standard concrete)

Ion Exchanger

\begin{tabular}{|l|c|c|c|c|c|c|c|c|c|c|}
\hline \multirow{2}{*}{ Isotope } & \multicolumn{8}{|c|}{ Location number (numbering as in Fig. 1) } \\
\cline { 2 - 12 } & $\mathbf{1}$ & $\mathbf{2}$ & $\mathbf{3}$ & $\mathbf{4}$ & $\mathbf{5}$ & $\mathbf{6}$ & $\mathbf{7}$ & $\mathbf{8}$ & $\mathbf{9}$ & $\mathbf{1 0}$ \\
\hline $\mathrm{Fe}-59$ & $9.50 \mathrm{E}-05$ & $3.27 \mathrm{E}-06$ & $3.44 \mathrm{E}-05$ & $5.14 \mathrm{E}-06$ & $3.10 \mathrm{E}-05$ & $1.08 \mathrm{E}-05$ & $1.44 \mathrm{E}-06$ & $9.20 \mathrm{E}-07$ & $9.10 \mathrm{E}-07$ & $2.18 \mathrm{E}-06$ \\
\hline $\mathrm{Mn}-54$ & $3.42 \mathrm{E}-05$ & $1.08 \mathrm{E}-06$ & $1.16 \mathrm{E}-05$ & $1.59 \mathrm{E}-06$ & $1.12 \mathrm{E}-05$ & $3.60 \mathrm{E}-06$ & $1.88 \mathrm{E}-07$ & $1.40 \mathrm{E}-07$ & $2.62 \mathrm{E}-07$ & $5.61 \mathrm{E}-07$ \\
\hline $\mathrm{Fe}-55$ & $1.60 \mathrm{E}-18$ & $1.73 \mathrm{E}-20$ & $2.46 \mathrm{E}-18$ & $1.27 \mathrm{E}-16$ & $1.17 \mathrm{E}-18$ & $6.07 \mathrm{E}-17$ & $3.27 \mathrm{E}-19$ & $7.38 \mathrm{E}-20$ & $1.30 \mathrm{E}-18$ & $1.81 \mathrm{E}-16$ \\
\hline $\mathrm{Co}-58$ & $3.46 \mathrm{E}-05$ & $1.11 \mathrm{E}-06$ & $1.17 \mathrm{E}-05$ & $1.58 \mathrm{E}-06$ & $1.13 \mathrm{E}-05$ & $3.62 \mathrm{E}-06$ & $2.78 \mathrm{E}-07$ & $1.94 \mathrm{E}-07$ & $2.85 \mathrm{E}-07$ & $5.66 \mathrm{E}-07$ \\
\hline $\mathrm{Co}-60$ & $2.22 \mathrm{E}-04$ & $7.81 \mathrm{E}-06$ & $8.11 \mathrm{E}-05$ & $1.22 \mathrm{E}-05$ & $7.24 \mathrm{E}-05$ & $2.54 \mathrm{E}-05$ & $2.44 \mathrm{E}-06$ & $1.54 \mathrm{E}-06$ & $1.92 \mathrm{E}-06$ & $5.27 \mathrm{E}-06$ \\
\hline $\mathrm{Cr}-51$ & $1.15 \mathrm{E}-07$ & $3.39 \mathrm{E}-09$ & $3.47 \mathrm{E}-08$ & $4.30 \mathrm{E}-09$ & $3.74 \mathrm{E}-08$ & $1.07 \mathrm{E}-08$ & $1.59 \mathrm{E}-10$ & $2.84 \mathrm{E}-10$ & $9.84 \mathrm{E}-10$ & $8.73 \mathrm{E}-10$ \\
\hline $\mathrm{Mn}-56$ & $1.69 \mathrm{E}-04$ & $7.32 \mathrm{E}-06$ & $8.09 \mathrm{E}-05$ & $1.50 \mathrm{E}-05$ & $5.95 \mathrm{E}-05$ & $2.47 \mathrm{E}-05$ & $1.71 \mathrm{E}-04$ & $1.08 \mathrm{E}-04$ & $4.73 \mathrm{E}-05$ & $6.51 \mathrm{E}-06$ \\
\hline $\mathrm{Cu}-64$ & $2.98 \mathrm{E}-06$ & $9.61 \mathrm{E}-08$ & $1.07 \mathrm{E}-06$ & $1.50 \mathrm{E}-07$ & $1.01 \mathrm{E}-06$ & $3.31 \mathrm{E}-07$ & $1.00 \mathrm{E}-06$ & $6.29 \mathrm{E}-07$ & $2.47 \mathrm{E}-07$ & $4.62 \mathrm{E}-08$ \\
\hline
\end{tabular}

Filter

\begin{tabular}{|l|c|c|c|c|c|c|c|c|c|c|}
\hline \multirow{2}{*}{ Isotope } & \multicolumn{10}{|c|}{ Location number (numbering as in Fig. 1) } \\
\cline { 2 - 12 } & $\mathbf{1}$ & $\mathbf{2}$ & $\mathbf{3}$ & $\mathbf{4}$ & $\mathbf{5}$ & $\mathbf{6}$ & $\mathbf{7}$ & $\mathbf{8}$ & $\mathbf{9}$ & $\mathbf{1 0}$ \\
\hline Fe-59 & $2.78 \mathrm{E}-06$ & $1.96 \mathrm{E}-07$ & $5.18 \mathrm{E}-05$ & $1.60 \mathrm{E}-03$ & $3.40 \mathrm{E}-05$ & $6.91 \mathrm{E}-04$ & $3.01 \mathrm{E}-06$ & $1.25 \mathrm{E}-06$ & $3.24 \mathrm{E}-05$ & $2.10 \mathrm{E}-03$ \\
\hline $\mathrm{Mn}-54$ & $1.18 \mathrm{E}-06$ & $8.76 \mathrm{E}-08$ & $1.81 \mathrm{E}-05$ & $6.31 \mathrm{E}-04$ & $1.19 \mathrm{E}-05$ & $2.77 \mathrm{E}-04$ & $1.23 \mathrm{E}-06$ & $5.44 \mathrm{E}-07$ & $1.18 \mathrm{E}-05$ & $8.44 \mathrm{E}-04$ \\
\hline Fe-55 & $5.17 \mathrm{E}-19$ & $2.86 \mathrm{E}-20$ & $4.64 \mathrm{E}-18$ & $2.54 \mathrm{E}-16$ & $2.39 \mathrm{E}-18$ & $1.21 \mathrm{E}-16$ & $5.28 \mathrm{E}-19$ & $1.67 \mathrm{E}-19$ & $2.68 \mathrm{E}-18$ & $3.66 \mathrm{E}-16$ \\
\hline $\mathrm{Co}-58$ & $1.24 \mathrm{E}-06$ & $9.44 \mathrm{E}-08$ & $1.85 \mathrm{E}-05$ & $6.49 \mathrm{E}-04$ & $1.21 \mathrm{E}-05$ & $2.85 \mathrm{E}-04$ & $1.29 \mathrm{E}-06$ & $5.64 \mathrm{E}-07$ & $1.21 \mathrm{E}-05$ & $8.69 \mathrm{E}-04$ \\
\hline Co-60 & $6.42 \mathrm{E}-06$ & $4.44 \mathrm{E}-07$ & $1.22 \mathrm{E}-04$ & $3.69 \mathrm{E}-03$ & $8.00 \mathrm{E}-05$ & $1.59 \mathrm{E}-03$ & $6.93 \mathrm{E}-06$ & $2.85 \mathrm{E}-06$ & $7.62 \mathrm{E}-05$ & $4.84 \mathrm{E}-03$ \\
\hline Cr-51 & $7.31 \mathrm{E}-09$ & $6.92 \mathrm{E}-10$ & $7.85 \mathrm{E}-08$ & $3.14 \mathrm{E}-06$ & $4.98 \mathrm{E}-08$ & $1.42 \mathrm{E}-06$ & $7.24 \mathrm{E}-09$ & $3.27 \mathrm{E}-09$ & $5.39 \mathrm{E}-08$ & $4.37 \mathrm{E}-06$ \\
\hline $\mathrm{Mn}-56$ & $5.44 \mathrm{E}-06$ & $3.08 \mathrm{E}-07$ & $1.15 \mathrm{E}-04$ & $2.98 \mathrm{E}-03$ & $7.44 \mathrm{E}-05$ & $1.26 \mathrm{E}-03$ & $5.95 \mathrm{E}-06$ & $2.32 \mathrm{E}-06$ & $6.99 \mathrm{E}-05$ & $3.86 \mathrm{E}-03$ \\
\hline $\mathrm{Cu}-64$ & $1.32 \mathrm{E}-07$ & $1.22 \mathrm{E}-08$ & $1.77 \mathrm{E}-06$ & $6.40 \mathrm{E}-05$ & $1.15 \mathrm{E}-06$ & $2.84 \mathrm{E}-05$ & $1.36 \mathrm{E}-07$ & $6.33 \mathrm{E}-08$ & $1.17 \mathrm{E}-06$ & $8.69 \mathrm{E}-05$ \\
\hline
\end{tabular}


Table 6. Dose Rates $(\mathrm{mSv} / \mathrm{h})$ for individual isotopes at external locations (30-cm thick wall, Barytes concrete)

Ion Exchanger

\begin{tabular}{|l|c|c|c|c|c|c|c|c|c|c|}
\hline \multirow{2}{*}{ Isotope } & \multicolumn{10}{|c|}{ Location number (numbering as in Fig. 1) } \\
\cline { 2 - 12 } & $\mathbf{1}$ & $\mathbf{2}$ & $\mathbf{3}$ & $\mathbf{4}$ & $\mathbf{5}$ & $\mathbf{6}$ & $\mathbf{7}$ & $\mathbf{8}$ & $\mathbf{9}$ & $\mathbf{1 0}$ \\
\hline Fe-59 & $9.31 \mathrm{E}-06$ & $1.49 \mathrm{E}-07$ & $2.81 \mathrm{E}-06$ & $2.83 \mathrm{E}-07$ & $3.14 \mathrm{E}-06$ & $9.01 \mathrm{E}-07$ & $1.20 \mathrm{E}-07$ & $7.58 \mathrm{E}-08$ & $5.66 \mathrm{E}-08$ & $1.35 \mathrm{E}-07$ \\
\hline $\mathrm{Mn}-54$ & $1.11 \mathrm{E}-05$ & $1.29 \mathrm{E}-07$ & $2.98 \mathrm{E}-06$ & $2.44 \mathrm{E}-07$ & $3.78 \mathrm{E}-06$ & $9.45 \mathrm{E}-07$ & $4.78 \mathrm{E}-08$ & $2.78 \mathrm{E}-08$ & $3.70 \mathrm{E}-08$ & $1.01 \mathrm{E}-07$ \\
\hline Fe-55 & $0.00 \mathrm{E}+00$ & $0.00 \mathrm{E}+00$ & $0.00 \mathrm{E}+00$ & $0.00 \mathrm{E}+00$ & $0.00 \mathrm{E}+00$ & $0.00 \mathrm{E}+00$ & $0.00 \mathrm{E}+00$ & $0.00 \mathrm{E}+00$ & $0.00 \mathrm{E}+00$ & $0.00 \mathrm{E}+00$ \\
\hline Co-58 & $1.62 \mathrm{E}-05$ & $2.01 \mathrm{E}-07$ & $4.43 \mathrm{E}-06$ & $3.75 \mathrm{E}-07$ & $5.51 \mathrm{E}-06$ & $1.38 \mathrm{E}-06$ & $1.31 \mathrm{E}-07$ & $7.93 \mathrm{E}-08$ & $6.91 \mathrm{E}-08$ & $1.51 \mathrm{E}-07$ \\
\hline Co-60 & $1.21 \mathrm{E}-04$ & $2.00 \mathrm{E}-06$ & $3.69 \mathrm{E}-05$ & $3.80 \mathrm{E}-06$ & $4.05 \mathrm{E}-05$ & $1.18 \mathrm{E}-05$ & $1.12 \mathrm{E}-06$ & $6.98 \mathrm{E}-07$ & $6.53 \mathrm{E}-07$ & $1.84 \mathrm{E}-06$ \\
\hline $\mathrm{Cr}-51$ & $8.45 \mathrm{E}-10$ & $8.43 \mathrm{E}-12$ & $1.30 \mathrm{E}-10$ & $1.04 \mathrm{E}-11$ & $3.85 \mathrm{E}-10$ & $2.46 \mathrm{E}-11$ & $0.00 \mathrm{E}+00$ & $0.00 \mathrm{E}+00$ & $2.72 \mathrm{E}-12$ & $0.00 \mathrm{E}+00$ \\
\hline Mn-56 & $9.13 \mathrm{E}-03$ & $2.24 \mathrm{E}-04$ & $3.92 \mathrm{E}-03$ & $5.52 \mathrm{E}-04$ & $3.32 \mathrm{E}-03$ & $1.23 \mathrm{E}-03$ & $8.58 \mathrm{E}-03$ & $5.38 \mathrm{E}-03$ & $2.16 \mathrm{E}-03$ & $2.36 \mathrm{E}-04$ \\
\hline Cu-64 & $1.31 \mathrm{E}-03$ & $1.94 \mathrm{E}-05$ & $4.10 \mathrm{E}-04$ & $4.41 \mathrm{E}-05$ & $4.62 \mathrm{E}-04$ & $1.33 \mathrm{E}-04$ & $3.99 \mathrm{E}-04$ & $2.57 \mathrm{E}-04$ & $9.53 \mathrm{E}-05$ & $1.67 \mathrm{E}-05$ \\
\hline TOTAL & $1.06 \mathrm{E}-02$ & $2.45 \mathrm{E}-04$ & $4.37 \mathrm{E}-03$ & $6.00 \mathrm{E}-04$ & $3.84 \mathrm{E}-03$ & $1.38 \mathrm{E}-03$ & $8.98 \mathrm{E}-03$ & $5.63 \mathrm{E}-03$ & $2.26 \mathrm{E}-03$ & $2.55 \mathrm{E}-04$ \\
\hline
\end{tabular}

Filter

\begin{tabular}{|l|c|c|c|c|c|c|c|c|c|c|}
\hline \multirow{2}{*}{ Isotope } & \multicolumn{10}{|c|}{ Location number (numbering as in Fig. 1) } \\
\cline { 2 - 12 } & $\mathbf{1}$ & $\mathbf{2}$ & $\mathbf{3}$ & $\mathbf{4}$ & $\mathbf{5}$ & $\mathbf{6}$ & $\mathbf{7}$ & $\mathbf{8}$ & $\mathbf{9}$ & $\mathbf{1 0}$ \\
\hline Fe-59 & $5.85 \mathrm{E}-09$ & $3.07 \mathrm{E}-10$ & $2.20 \mathrm{E}-07$ & $1.39 \mathrm{E}-05$ & $1.58 \mathrm{E}-07$ & $6.50 \mathrm{E}-06$ & $6.95 \mathrm{E}-09$ & $2.45 \mathrm{E}-09$ & $1.36 \mathrm{E}-07$ & $1.78 \mathrm{E}-05$ \\
\hline $\mathrm{Mn}-54$ & $6.02 \mathrm{E}-09$ & $3.53 \mathrm{E}-10$ & $2.20 \mathrm{E}-07$ & $1.93 \mathrm{E}-05$ & $1.60 \mathrm{E}-07$ & $9.37 \mathrm{E}-06$ & $7.37 \mathrm{E}-09$ & $2.88 \mathrm{E}-09$ & $1.41 \mathrm{E}-07$ & $2.48 \mathrm{E}-05$ \\
\hline Fe-55 & $0.00 \mathrm{E}+00$ & $0.00 \mathrm{E}+00$ & $0.00 \mathrm{E}+00$ & $0.00 \mathrm{E}+00$ & $0.00 \mathrm{E}+00$ & $0.00 \mathrm{E}+00$ & $0.00 \mathrm{E}+00$ & $0.00 \mathrm{E}+00$ & $0.00 \mathrm{E}+00$ & $0.00 \mathrm{E}+00$ \\
\hline Co-58 & $9.72 \mathrm{E}-09$ & $6.12 \mathrm{E}-10$ & $3.36 \mathrm{E}-07$ & $2.85 \mathrm{E}-05$ & $2.43 \mathrm{E}-07$ & $1.38 \mathrm{E}-05$ & $1.06 \mathrm{E}-08$ & $4.57 \mathrm{E}-09$ & $2.17 \mathrm{E}-07$ & $3.66 \mathrm{E}-05$ \\
\hline Co-60 & $8.38 \mathrm{E}-08$ & $4.47 \mathrm{E}-09$ & $3.19 \mathrm{E}-06$ & $1.93 \mathrm{E}-04$ & $2.27 \mathrm{E}-06$ & $8.98 \mathrm{E}-05$ & $9.98 \mathrm{E}-08$ & $3.52 \mathrm{E}-08$ & $1.95 \mathrm{E}-06$ & $2.46 \mathrm{E}-04$ \\
\hline $\mathrm{Cr}-51$ & $0.00 \mathrm{E}+00$ & $0.00 \mathrm{E}+00$ & $4.70 \mathrm{E}-12$ & $2.11 \mathrm{E}-09$ & $2.26 \mathrm{E}-12$ & $1.23 \mathrm{E}-09$ & $0.00 \mathrm{E}+00$ & $0.00 \mathrm{E}+00$ & $4.12 \mathrm{E}-12$ & $2.71 \mathrm{E}-09$ \\
\hline $\mathrm{Mn}-56$ & $6.59 \mathrm{E}-06$ & $2.89 \mathrm{E}-07$ & $2.69 \mathrm{E}-04$ & $1.21 \mathrm{E}-02$ & $1.88 \mathrm{E}-04$ & $5.47 \mathrm{E}-03$ & $7.87 \mathrm{E}-06$ & $2.57 \mathrm{E}-06$ & $1.59 \mathrm{E}-04$ & $1.53 \mathrm{E}-02$ \\
\hline Cu-64 & $8.22 \mathrm{E}-07$ & $4.07 \mathrm{E}-08$ & $2.99 \mathrm{E}-05$ & $2.14 \mathrm{E}-03$ & $2.13 \mathrm{E}-05$ & $1.02 \mathrm{E}-03$ & $8.65 \mathrm{E}-07$ & $3.17 \mathrm{E}-07$ & $1.86 \mathrm{E}-05$ & $2.74 \mathrm{E}-03$ \\
\hline TOTAL & $7.52 \mathrm{E}-06$ & $3.36 \mathrm{E}-07$ & $3.02 \mathrm{E}-04$ & $1.45 \mathrm{E}-02$ & $2.12 \mathrm{E}-04$ & $6.61 \mathrm{E}-03$ & $8.86 \mathrm{E}-06$ & $2.94 \mathrm{E}-06$ & $1.80 \mathrm{E}-04$ & $1.83 \mathrm{E}-02$ \\
\hline
\end{tabular}

Ion Exchanger + Filter

\begin{tabular}{|l|c|c|c|c|c|c|c|c|c|c|}
\hline \multirow{2}{*}{ Isotope } & \multicolumn{10}{|c|}{ Location number (numbering as in Fig. 1) } \\
\cline { 2 - 11 } & $\mathbf{1}$ & $\mathbf{2}$ & $\mathbf{3}$ & $\mathbf{4}$ & $\mathbf{5}$ & $\mathbf{6}$ & $\mathbf{7}$ & $\mathbf{8}$ & $\mathbf{9}$ & $\mathbf{1 0}$ \\
\hline Fe-59 & $9.31 \mathrm{E}-06$ & $1.49 \mathrm{E}-07$ & $3.03 \mathrm{E}-06$ & $1.42 \mathrm{E}-05$ & $3.30 \mathrm{E}-06$ & $7.40 \mathrm{E}-06$ & $1.27 \mathrm{E}-07$ & $7.82 \mathrm{E}-08$ & $1.92 \mathrm{E}-07$ & $1.79 \mathrm{E}-05$ \\
\hline Mn-54 & $1.11 \mathrm{E}-05$ & $1.29 \mathrm{E}-07$ & $3.20 \mathrm{E}-06$ & $1.96 \mathrm{E}-05$ & $3.94 \mathrm{E}-06$ & $1.03 \mathrm{E}-05$ & $5.52 \mathrm{E}-08$ & $3.07 \mathrm{E}-08$ & $1.78 \mathrm{E}-07$ & $2.49 \mathrm{E}-05$ \\
\hline Fe-55 & $0.00 \mathrm{E}+00$ & $0.00 \mathrm{E}+00$ & $0.00 \mathrm{E}+00$ & $0.00 \mathrm{E}+00$ & $0.00 \mathrm{E}+00$ & $0.00 \mathrm{E}+00$ & $0.00 \mathrm{E}+00$ & $0.00 \mathrm{E}+00$ & $0.00 \mathrm{E}+00$ & $0.00 \mathrm{E}+00$ \\
\hline Co-58 & $1.62 \mathrm{E}-05$ & $2.01 \mathrm{E}-07$ & $4.77 \mathrm{E}-06$ & $2.89 \mathrm{E}-05$ & $5.76 \mathrm{E}-06$ & $1.52 \mathrm{E}-05$ & $1.42 \mathrm{E}-07$ & $8.39 \mathrm{E}-08$ & $2.86 \mathrm{E}-07$ & $3.67 \mathrm{E}-05$ \\
\hline Co-60 & $1.21 \mathrm{E}-04$ & $2.01 \mathrm{E}-06$ & $4.01 \mathrm{E}-05$ & $1.97 \mathrm{E}-04$ & $4.28 \mathrm{E}-05$ & $1.02 \mathrm{E}-04$ & $1.22 \mathrm{E}-06$ & $7.33 \mathrm{E}-07$ & $2.60 \mathrm{E}-06$ & $2.48 \mathrm{E}-04$ \\
\hline Cr-51 & $8.45 \mathrm{E}-10$ & $8.43 \mathrm{E}-12$ & $1.35 \mathrm{E}-10$ & $2.12 \mathrm{E}-09$ & $3.87 \mathrm{E}-10$ & $1.26 \mathrm{E}-09$ & $0.00 \mathrm{E}+00$ & $0.00 \mathrm{E}+00$ & $6.84 \mathrm{E}-12$ & $2.71 \mathrm{E}-09$ \\
\hline Mn-56 & $9.13 \mathrm{E}-03$ & $2.24 \mathrm{E}-04$ & $4.18 \mathrm{E}-03$ & $1.27 \mathrm{E}-02$ & $3.51 \mathrm{E}-03$ & $6.70 \mathrm{E}-03$ & $8.59 \mathrm{E}-03$ & $5.38 \mathrm{E}-03$ & $2.32 \mathrm{E}-03$ & $1.55 \mathrm{E}-02$ \\
\hline Cu-64 & $1.31 \mathrm{E}-03$ & $1.94 \mathrm{E}-05$ & $4.39 \mathrm{E}-04$ & $2.19 \mathrm{E}-03$ & $4.84 \mathrm{E}-04$ & $1.16 \mathrm{E}-03$ & $4.00 \mathrm{E}-04$ & $2.57 \mathrm{E}-04$ & $1.14 \mathrm{E}-04$ & $2.75 \mathrm{E}-03$ \\
\hline TOTAL & $1.06 \mathrm{E}-02$ & $2.46 \mathrm{E}-04$ & $4.67 \mathrm{E}-03$ & $1.51 \mathrm{E}-02$ & $4.05 \mathrm{E}-03$ & $7.99 \mathrm{E}-03$ & $8.99 \mathrm{E}-03$ & $5.64 \mathrm{E}-03$ & $2.44 \mathrm{E}-03$ & $1.86 \mathrm{E}-02$ \\
\hline
\end{tabular}


Table 7. Conversion factors ( $\mathrm{mSv} / \mathrm{h}$-per-GBq) for individual isotopes at external locations (30-cm thick wall, standard concrete)

Ion Exchanger

\begin{tabular}{|l|c|c|c|c|c|c|c|c|c|c|}
\hline \multirow{2}{*}{ Isotope } & \multicolumn{10}{|c|}{ Location number (numbering as in Fig. 1) } \\
\cline { 2 - 12 } & $\mathbf{1}$ & $\mathbf{2}$ & $\mathbf{3}$ & $\mathbf{4}$ & $\mathbf{5}$ & $\mathbf{6}$ & $\mathbf{7}$ & $\mathbf{8}$ & $\mathbf{9}$ & $\mathbf{1 0}$ \\
\hline $\mathrm{Fe}-59$ & $1.72 \mathrm{E}-05$ & $2.76 \mathrm{E}-07$ & $5.20 \mathrm{E}-06$ & $5.24 \mathrm{E}-07$ & $5.81 \mathrm{E}-06$ & $1.67 \mathrm{E}-06$ & $2.22 \mathrm{E}-07$ & $1.40 \mathrm{E}-07$ & $1.05 \mathrm{E}-07$ & $2.49 \mathrm{E}-07$ \\
\hline $\mathrm{Mn}-54$ & $3.22 \mathrm{E}-06$ & $3.75 \mathrm{E}-08$ & $8.67 \mathrm{E}-07$ & $7.11 \mathrm{E}-08$ & $1.10 \mathrm{E}-06$ & $2.75 \mathrm{E}-07$ & $1.39 \mathrm{E}-08$ & $8.10 \mathrm{E}-09$ & $1.08 \mathrm{E}-08$ & $2.95 \mathrm{E}-08$ \\
\hline $\mathrm{Fe}-55$ & $0.00 \mathrm{E}+00$ & $0.00 \mathrm{E}+00$ & $0.00 \mathrm{E}+00$ & $0.00 \mathrm{E}+00$ & $0.00 \mathrm{E}+00$ & $0.00 \mathrm{E}+00$ & $0.00 \mathrm{E}+00$ & $0.00 \mathrm{E}+00$ & $0.00 \mathrm{E}+00$ & $0.00 \mathrm{E}+00$ \\
\hline $\mathrm{Co}-58$ & $3.13 \mathrm{E}-06$ & $3.87 \mathrm{E}-08$ & $8.55 \mathrm{E}-07$ & $7.24 \mathrm{E}-08$ & $1.06 \mathrm{E}-06$ & $2.65 \mathrm{E}-07$ & $2.53 \mathrm{E}-08$ & $1.53 \mathrm{E}-08$ & $1.33 \mathrm{E}-08$ & $2.91 \mathrm{E}-08$ \\
\hline $\mathrm{Co}-60$ & $4.32 \mathrm{E}-05$ & $7.17 \mathrm{E}-07$ & $1.32 \mathrm{E}-05$ & $1.36 \mathrm{E}-06$ & $1.45 \mathrm{E}-05$ & $4.22 \mathrm{E}-06$ & $4.02 \mathrm{E}-07$ & $2.50 \mathrm{E}-07$ & $2.34 \mathrm{E}-07$ & $6.59 \mathrm{E}-07$ \\
\hline $\mathrm{Cr}-51$ & $4.74 \mathrm{E}-11$ & $4.73 \mathrm{E}-13$ & $7.30 \mathrm{E}-12$ & $5.83 \mathrm{E}-13$ & $2.16 \mathrm{E}-11$ & $1.38 \mathrm{E}-12$ & $0.00 \mathrm{E}+00$ & $0.00 \mathrm{E}+00$ & $1.52 \mathrm{E}-13$ & $0.00 \mathrm{E}+00$ \\
\hline $\mathrm{Mn}-56$ & $4.28 \mathrm{E}-05$ & $1.05 \mathrm{E}-06$ & $1.84 \mathrm{E}-05$ & $2.59 \mathrm{E}-06$ & $1.56 \mathrm{E}-05$ & $5.78 \mathrm{E}-06$ & $4.03 \mathrm{E}-05$ & $2.52 \mathrm{E}-05$ & $1.02 \mathrm{E}-05$ & $1.11 \mathrm{E}-06$ \\
\hline $\mathrm{Cu}-64$ & $1.71 \mathrm{E}-07$ & $2.53 \mathrm{E}-09$ & $5.34 \mathrm{E}-08$ & $5.75 \mathrm{E}-09$ & $6.03 \mathrm{E}-08$ & $1.74 \mathrm{E}-08$ & $5.21 \mathrm{E}-08$ & $3.35 \mathrm{E}-08$ & $1.24 \mathrm{E}-08$ & $2.17 \mathrm{E}-09$ \\
\hline
\end{tabular}

Filter

\begin{tabular}{|l|c|c|c|c|c|c|c|c|c|c|}
\hline \multirow{2}{*}{ Isotope } & \multicolumn{9}{|c|}{ Location number (numbering as in Fig. 1) } \\
\cline { 2 - 12 } & $\mathbf{1}$ & $\mathbf{2}$ & $\mathbf{3}$ & $\mathbf{4}$ & $\mathbf{5}$ & $\mathbf{6}$ & $\mathbf{7}$ & $\mathbf{8}$ & $\mathbf{9}$ & $\mathbf{1 0}$ \\
\hline $\mathrm{Fe}-59$ & $1.17 \mathrm{E}-07$ & $6.15 \mathrm{E}-09$ & $4.40 \mathrm{E}-06$ & $2.79 \mathrm{E}-04$ & $3.16 \mathrm{E}-06$ & $1.30 \mathrm{E}-04$ & $1.39 \mathrm{E}-07$ & $4.89 \mathrm{E}-08$ & $2.71 \mathrm{E}-06$ & $3.55 \mathrm{E}-04$ \\
\hline $\mathrm{Mn}-54$ & $1.77 \mathrm{E}-08$ & $1.04 \mathrm{E}-09$ & $6.47 \mathrm{E}-07$ & $5.69 \mathrm{E}-05$ & $4.71 \mathrm{E}-07$ & $2.76 \mathrm{E}-05$ & $2.17 \mathrm{E}-08$ & $8.48 \mathrm{E}-09$ & $4.16 \mathrm{E}-07$ & $7.30 \mathrm{E}-05$ \\
\hline $\mathrm{Fe}-55$ & $0.00 \mathrm{E}+00$ & $0.00 \mathrm{E}+00$ & $0.00 \mathrm{E}+00$ & $0.00 \mathrm{E}+00$ & $0.00 \mathrm{E}+00$ & $0.00 \mathrm{E}+00$ & $0.00 \mathrm{E}+00$ & $0.00 \mathrm{E}+00$ & $0.00 \mathrm{E}+00$ & $0.00 \mathrm{E}+00$ \\
\hline $\mathrm{Co}-58$ & $1.87 \mathrm{E}-08$ & $1.18 \mathrm{E}-09$ & $6.46 \mathrm{E}-07$ & $5.48 \mathrm{E}-05$ & $4.67 \mathrm{E}-07$ & $2.65 \mathrm{E}-05$ & $2.03 \mathrm{E}-08$ & $8.78 \mathrm{E}-09$ & $4.17 \mathrm{E}-07$ & $7.03 \mathrm{E}-05$ \\
\hline $\mathrm{Co}-60$ & $2.99 \mathrm{E}-07$ & $1.59 \mathrm{E}-08$ & $1.14 \mathrm{E}-05$ & $6.91 \mathrm{E}-04$ & $8.10 \mathrm{E}-06$ & $3.21 \mathrm{E}-04$ & $3.56 \mathrm{E}-07$ & $1.26 \mathrm{E}-07$ & $6.97 \mathrm{E}-06$ & $8.80 \mathrm{E}-04$ \\
\hline $\mathrm{Cr}-51$ & $0.00 \mathrm{E}+00$ & $0.00 \mathrm{E}+00$ & $2.64 \mathrm{E}-12$ & $1.18 \mathrm{E}-09$ & $1.27 \mathrm{E}-12$ & $6.93 \mathrm{E}-10$ & $0.00 \mathrm{E}+00$ & $0.00 \mathrm{E}+00$ & $2.32 \mathrm{E}-12$ & $1.52 \mathrm{E}-09$ \\
\hline $\mathrm{Mn}-56$ & $3.89 \mathrm{E}-07$ & $1.71 \mathrm{E}-08$ & $1.59 \mathrm{E}-05$ & $7.17 \mathrm{E}-04$ & $1.11 \mathrm{E}-05$ & $3.23 \mathrm{E}-04$ & $4.65 \mathrm{E}-07$ & $1.52 \mathrm{E}-07$ & $9.37 \mathrm{E}-06$ & $9.02 \mathrm{E}-04$ \\
\hline $\mathrm{Cu}-64$ & $1.16 \mathrm{E}-09$ & $5.75 \mathrm{E}-11$ & $4.22 \mathrm{E}-08$ & $3.03 \mathrm{E}-06$ & $3.01 \mathrm{E}-08$ & $1.45 \mathrm{E}-06$ & $1.22 \mathrm{E}-09$ & $4.48 \mathrm{E}-10$ & $2.63 \mathrm{E}-08$ & $3.86 \mathrm{E}-06$ \\
\hline
\end{tabular}

Table 8. Decay factors for source isotopes

\begin{tabular}{|l|c|c|c|c|}
\hline \multirow{2}{*}{ Isotope } & \multirow{2}{*}{ Half-life } & \multicolumn{3}{|c|}{ Decay factor following time from shutdown } \\
\cline { 3 - 5 } & & 0 days (shutdown) & $\mathbf{2}$ days & $\mathbf{1 0}$ days \\
\hline Fe-59 & $44.5 \mathrm{~d}$ & 1 & $9.693 \mathrm{E}-01$ & $8.558 \mathrm{E}-01$ \\
\hline $\mathrm{Mn}-54$ & $312.1 \mathrm{~d}$ & 1 & $9.956 \mathrm{E}-01$ & $9.780 \mathrm{E}-01$ \\
\hline Fe-55 & $2.73 \mathrm{y}$ & 1 & $9.986 \mathrm{E}-01$ & $9.931 \mathrm{E}-01$ \\
\hline $\mathrm{Co}-58$ & $70.88 \mathrm{~d}$ & 1 & $9.806 \mathrm{E}-01$ & $9.068 \mathrm{E}-01$ \\
\hline $\mathrm{Co}-60$ & $5.271 \mathrm{y}$ & 1 & $9.993 \mathrm{E}-01$ & $9.964 \mathrm{E}-01$ \\
\hline $\mathrm{Cr}-51$ & $27.702 \mathrm{~d}$ & 1 & $9.512 \mathrm{E}-01$ & $7.786 \mathrm{E}-01$ \\
\hline $\mathrm{Mn}-56$ & $2.578 \mathrm{~h}$ & 1 & $2.484 \mathrm{E}-06$ & $9.451 \mathrm{E}-29$ \\
\hline $\mathrm{Cu}-64$ & $12.701 \mathrm{~h}$ & 1 & $7.283 \mathrm{E}-02$ & $2.050 \mathrm{E}-06$ \\
\hline
\end{tabular}


Table 9. Dose rates $(\mathrm{mSv} / \mathrm{h})$ for individual isotopes at $5 \mathrm{~cm}$ from the equipment outer surface at shutdown, 2 days, and 10 days after shutdown

Ion Exchanger

\begin{tabular}{|c|c|c|c|c|c|c|}
\hline \multirow{3}{*}{ Isotope } & \multicolumn{6}{|c|}{ Dose rates, $\mathrm{mSv} / \mathrm{h}$} \\
\hline & \multicolumn{3}{|c|}{ Horizontal Axis $5 \mathrm{~cm}$} & \multicolumn{3}{|c|}{ Vertical Axis $5 \mathrm{~cm}$} \\
\hline & Shutdown & 2 days & 10 days & Shutdown & 2 days & 10 days \\
\hline Fe-59 & $6.88 \mathrm{E}-03$ & $6.66 \mathrm{E}-03$ & $5.88 \mathrm{E}-03$ & $4.22 \mathrm{E}-05$ & $4.09 \mathrm{E}-05$ & $3.62 \mathrm{E}-05$ \\
\hline $\mathrm{Mn}-54$ & $2.58 \mathrm{E}-02$ & $2.57 \mathrm{E}-02$ & $2.52 \mathrm{E}-02$ & $5.99 \mathrm{E}-05$ & $5.97 \mathrm{E}-05$ & $5.86 \mathrm{E}-05$ \\
\hline Fe-55 & $6.52 \mathrm{E}-12$ & $6.51 \mathrm{E}-12$ & $6.48 \mathrm{E}-12$ & $3.06 \mathrm{E}-12$ & $3.06 \mathrm{E}-12$ & $3.04 \mathrm{E}-12$ \\
\hline Co-58 & $4.29 \mathrm{E}-02$ & $4.21 \mathrm{E}-02$ & $3.89 \mathrm{E}-02$ & $1.35 \mathrm{E}-04$ & $1.33 \mathrm{E}-04$ & $1.23 \mathrm{E}-04$ \\
\hline Co- 60 & $7.73 \mathrm{E}-02$ & $7.72 \mathrm{E}-02$ & $7.70 \mathrm{E}-02$ & $3.35 \mathrm{E}-04$ & $3.35 \mathrm{E}-04$ & $3.34 \mathrm{E}-04$ \\
\hline $\mathrm{Cr}-51$ & $2.19 \mathrm{E}-03$ & $2.08 \mathrm{E}-03$ & $1.71 \mathrm{E}-03$ & $1.28 \mathrm{E}-06$ & $1.21 \mathrm{E}-06$ & $9.94 \mathrm{E}-07$ \\
\hline Mn-56 & $3.19 \mathrm{E}+00$ & $7.93 \mathrm{E}-06$ & $3.02 \mathrm{E}-28$ & $1.52 \mathrm{E}+00$ & $3.78 \mathrm{E}-06$ & $1.44 \mathrm{E}-28$ \\
\hline $\mathrm{Cu}-64$ & $8.76 \mathrm{E}+00$ & $6.38 \mathrm{E}-01$ & $1.80 \mathrm{E}-05$ & $1.36 \mathrm{E}+00$ & $9.93 \mathrm{E}-02$ & $2.79 \mathrm{E}-06$ \\
\hline TOTAL & $1.21 \mathrm{E}+01$ & $7.92 \mathrm{E}-01$ & $1.49 \mathrm{E}-01$ & $2.88 \mathrm{E}+00$ & $9.99 \mathrm{E}-02$ & $5.55 \mathrm{E}-04$ \\
\hline
\end{tabular}

Filter

\begin{tabular}{|c|c|c|c|c|c|c|}
\hline \multirow{3}{*}{ Isotope } & \multicolumn{6}{|c|}{ Dose rates, $\mathrm{mSv} / \mathrm{h}$} \\
\hline & \multicolumn{3}{|c|}{ Horizontal Axis $5 \mathrm{~cm}$} & \multicolumn{3}{|c|}{ Vertical Axis $5 \mathrm{~cm}$} \\
\hline & Shutdown & 2 days & 10 days & Shutdown & 2 days & 10 days \\
\hline $\mathrm{Fe}-59$ & $6.41 \mathrm{E}-03$ & $6.21 \mathrm{E}-03$ & 5.49E-03 & $7.80 \mathrm{E}-03$ & $7.56 \mathrm{E}-03$ & 6.67E-03 \\
\hline $\mathrm{Mn}-54$ & $2.86 \mathrm{E}-02$ & $2.85 \mathrm{E}-02$ & $2.80 \mathrm{E}-02$ & $3.51 \mathrm{E}-02$ & $3.50 \mathrm{E}-02$ & $3.44 \mathrm{E}-02$ \\
\hline $\mathrm{Fe}-55$ & $3.88 \mathrm{E}-13$ & $3.87 \mathrm{E}-13$ & $3.85 \mathrm{E}-13$ & 4.62E-13 & $4.61 \mathrm{E}-13$ & $4.59 \mathrm{E}-13$ \\
\hline $\mathrm{Co}-58$ & 4.97E-02 & $4.88 \mathrm{E}-02$ & $4.51 \mathrm{E}-02$ & $6.11 \mathrm{E}-02$ & $5.99 \mathrm{E}-02$ & 5.54E-02 \\
\hline Co-60 & $7.68 \mathrm{E}-02$ & $7.67 \mathrm{E}-02$ & $7.65 \mathrm{E}-02$ & $9.33 \mathrm{E}-02$ & $9.32 \mathrm{E}-02$ & 9.29E-02 \\
\hline $\mathrm{Cr}-51$ & $3.89 \mathrm{E}-03$ & $3.70 \mathrm{E}-03$ & $3.03 \mathrm{E}-03$ & 4.80E-03 & $4.56 \mathrm{E}-03$ & $3.74 \mathrm{E}-03$ \\
\hline $\mathrm{Mn}-56$ & $3.14 \mathrm{E}+00$ & $7.81 \mathrm{E}-06$ & $2.97 \mathrm{E}-28$ & $3.79 \mathrm{E}+00$ & $9.41 \mathrm{E}-06$ & $3.58 \mathrm{E}-28$ \\
\hline $\mathrm{Cu}-64$ & $1.17 \mathrm{E}+01$ & 8.49E-01 & 2.39E-05 & $1.44 \mathrm{E}+01$ & $1.05 \mathrm{E}+00$ & $2.95 \mathrm{E}-05$ \\
\hline TOTAL & $1.50 \mathrm{E}+01$ & $1.01 E+00$ & $1.58 \mathrm{E}-01$ & $1.84 \mathrm{E}+01$ & $1.25 \mathrm{E}+00$ & 1.93E-01 \\
\hline
\end{tabular}


Table 10. Dose rates $(\mathrm{mSv} / \mathrm{h})$ for individual isotopes at $30 \mathrm{~cm}$ from the equipment outer surface at shutdown, 2 days, and 10 days after shutdown

Ion Exchanger

\begin{tabular}{|c|c|c|c|c|c|c|}
\hline \multirow{3}{*}{ Isotope } & \multicolumn{6}{|c|}{ Dose rates, $\mathrm{mSv} / \mathrm{h}$} \\
\hline & \multicolumn{3}{|c|}{ Horizontal Axis $30 \mathrm{~cm}$} & \multicolumn{3}{|c|}{ Vertical Axis $30 \mathrm{~cm}$} \\
\hline & Shutdown & 2 days & 10 days & Shutdown & 2 days & 10 days \\
\hline Fe-59 & $4.71 \mathrm{E}-03$ & $4.57 \mathrm{E}-03$ & $4.03 \mathrm{E}-03$ & $3.92 \mathrm{E}-05$ & $3.80 \mathrm{E}-05$ & $3.36 \mathrm{E}-05$ \\
\hline $\mathrm{Mn}-54$ & $1.79 \mathrm{E}-02$ & $1.78 \mathrm{E}-02$ & $1.75 \mathrm{E}-02$ & $5.70 \mathrm{E}-05$ & $5.68 \mathrm{E}-05$ & $5.58 \mathrm{E}-05$ \\
\hline $\mathrm{Fe}-55$ & $6.95 \mathrm{E}-12$ & $6.94 \mathrm{E}-12$ & $6.90 \mathrm{E}-12$ & $4.18 \mathrm{E}-12$ & 4.17E-12 & $4.15 \mathrm{E}-12$ \\
\hline Co-58 & $2.98 \mathrm{E}-02$ & $2.92 \mathrm{E}-02$ & $2.70 \mathrm{E}-02$ & $1.31 \mathrm{E}-04$ & $1.28 \mathrm{E}-04$ & $1.18 \mathrm{E}-04$ \\
\hline Co-60 & $5.29 \mathrm{E}-02$ & $5.28 \mathrm{E}-02$ & $5.27 \mathrm{E}-02$ & $3.05 \mathrm{E}-04$ & $3.05 \mathrm{E}-04$ & $3.04 \mathrm{E}-04$ \\
\hline $\mathrm{Cr}-51$ & $1.57 \mathrm{E}-03$ & $1.50 \mathrm{E}-03$ & $1.23 \mathrm{E}-03$ & $1.31 \mathrm{E}-06$ & $1.25 \mathrm{E}-06$ & $1.02 \mathrm{E}-06$ \\
\hline Mn-56 & $2.23 \mathrm{E}+00$ & $5.54 \mathrm{E}-06$ & $2.11 \mathrm{E}-28$ & $1.39 \mathrm{E}+00$ & $3.46 \mathrm{E}-06$ & $1.32 \mathrm{E}-28$ \\
\hline $\mathrm{Cu}-64$ & $6.19 \mathrm{E}+00$ & $4.51 \mathrm{E}-01$ & $1.27 \mathrm{E}-05$ & $1.25 \mathrm{E}+00$ & $9.09 \mathrm{E}-02$ & $2.56 \mathrm{E}-06$ \\
\hline TOTAL & $8.52 E+00$ & 5.57E-01 & 1.02E-01 & $2.64 E+00$ & $9.14 \mathrm{E}-02$ & $5.16 \mathrm{E}-04$ \\
\hline
\end{tabular}

Filter

\begin{tabular}{|c|c|c|c|c|c|c|}
\hline \multirow{3}{*}{ Isotope } & \multicolumn{6}{|c|}{ Dose rates, $\mathrm{mSv} / \mathrm{h}$} \\
\hline & \multicolumn{3}{|c|}{ Horizontal Axis $30 \mathrm{~cm}$} & \multicolumn{3}{|c|}{ Vertical Axis $30 \mathrm{~cm}$} \\
\hline & Shutdown & 2 days & 10 days & Shutdown & 2 days & 10 days \\
\hline Fe-59 & $3.31 \mathrm{E}-03$ & $3.21 \mathrm{E}-03$ & $2.84 \mathrm{E}-03$ & $3.91 \mathrm{E}-03$ & $3.79 \mathrm{E}-03$ & $3.34 \mathrm{E}-03$ \\
\hline $\mathrm{Mn}-54$ & $1.50 \mathrm{E}-02$ & $1.49 \mathrm{E}-02$ & $1.46 \mathrm{E}-02$ & $1.83 \mathrm{E}-02$ & $1.82 \mathrm{E}-02$ & $1.79 \mathrm{E}-02$ \\
\hline $\mathrm{Fe}-55$ & $2.26 \mathrm{E}-13$ & $2.25 \mathrm{E}-13$ & $2.24 \mathrm{E}-13$ & $3.38 \mathrm{E}-13$ & $3.37 \mathrm{E}-13$ & $3.36 \mathrm{E}-13$ \\
\hline Co-58 & $2.61 \mathrm{E}-02$ & $2.56 \mathrm{E}-02$ & $2.37 \mathrm{E}-02$ & $3.21 \mathrm{E}-02$ & $3.15 \mathrm{E}-02$ & $2.91 \mathrm{E}-02$ \\
\hline Co-60 & $3.96 \mathrm{E}-02$ & $3.95 \mathrm{E}-02$ & $3.94 \mathrm{E}-02$ & 4.64E-02 & 4.64E-02 & 4.62E-02 \\
\hline $\mathrm{Cr}-51$ & $2.11 \mathrm{E}-03$ & $2.01 \mathrm{E}-03$ & $1.64 \mathrm{E}-03$ & $2.80 \mathrm{E}-03$ & $2.67 \mathrm{E}-03$ & $2.18 \mathrm{E}-03$ \\
\hline $\mathrm{Mn}-56$ & $1.61 \mathrm{E}+00$ & $4.00 \mathrm{E}-06$ & $1.52 \mathrm{E}-28$ & $1.88 \mathrm{E}+00$ & $4.67 \mathrm{E}-06$ & $1.78 \mathrm{E}-28$ \\
\hline $\mathrm{Cu}-64$ & $6.22 \mathrm{E}+00$ & $4.53 \mathrm{E}-01$ & $1.27 \mathrm{E}-05$ & $7.88 \mathrm{E}+00$ & $5.74 \mathrm{E}-01$ & $1.62 \mathrm{E}-05$ \\
\hline TOTAL & $7.91 \mathrm{E}+00$ & $5.38 \mathrm{E}-01$ & $8.22 \mathrm{E}-02$ & $9.87 E+00$ & $6.77 E-01$ & $9.88 \mathrm{E}-02$ \\
\hline
\end{tabular}


Table 11. Dose rates $(\mathrm{mSv} / \mathrm{h})$ for individual isotopes at $50 \mathrm{~cm}$ from the equipment outer surface at shutdown, 2 days, and 10 days after shutdown

Ion Exchanger

\begin{tabular}{|l|c|c|c|c|c|c|}
\hline \multirow{3}{*}{ Isotope } & \multicolumn{6}{c|}{ Dose rates, mSv/h } \\
\cline { 2 - 8 } & \multicolumn{2}{|c|}{ Horizontal Axis 50 cm } & \multicolumn{3}{c|}{ Vertical Axis 50 cm } \\
\cline { 2 - 8 } & Shutdown & 2 days & 10 days & Shutdown & 2 days & 10 days \\
\hline Fe-59 & $3.48 \mathrm{E}-03$ & $3.37 \mathrm{E}-03$ & $2.98 \mathrm{E}-03$ & $3.39 \mathrm{E}-05$ & $3.28 \mathrm{E}-05$ & $2.90 \mathrm{E}-05$ \\
\hline Mn-54 & $1.33 \mathrm{E}-02$ & $1.33 \mathrm{E}-02$ & $1.30 \mathrm{E}-02$ & $4.79 \mathrm{E}-05$ & $4.77 \mathrm{E}-05$ & $4.68 \mathrm{E}-05$ \\
\hline Fe-55 & $7.38 \mathrm{E}-12$ & $7.37 \mathrm{E}-12$ & $7.33 \mathrm{E}-12$ & $4.11 \mathrm{E}-12$ & $4.10 \mathrm{E}-12$ & $4.08 \mathrm{E}-12$ \\
\hline Co-58 & $2.23 \mathrm{E}-02$ & $2.19 \mathrm{E}-02$ & $2.02 \mathrm{E}-02$ & $1.08 \mathrm{E}-04$ & $1.06 \mathrm{E}-04$ & $9.82 \mathrm{E}-05$ \\
\hline Co-60 & $3.90 \mathrm{E}-02$ & $3.90 \mathrm{E}-02$ & $3.89 \mathrm{E}-02$ & $2.65 \mathrm{E}-04$ & $2.65 \mathrm{E}-04$ & $2.64 \mathrm{E}-04$ \\
\hline Cr-51 & $1.20 \mathrm{E}-03$ & $1.14 \mathrm{E}-03$ & $9.35 \mathrm{E}-04$ & $1.18 \mathrm{E}-06$ & $1.12 \mathrm{E}-06$ & $9.19 \mathrm{E}-07$ \\
\hline Mn-56 & $1.68 \mathrm{E}+00$ & $4.17 \mathrm{E}-06$ & $1.59 \mathrm{E}-28$ & $1.23 \mathrm{E}+00$ & $3.05 \mathrm{E}-06$ & $1.16 \mathrm{E}-28$ \\
\hline Cu-64 & $4.71 \mathrm{E}+00$ & $3.43 \mathrm{E}-01$ & $9.65 \mathrm{E}-06$ & $1.13 \mathrm{E}+00$ & $8.24 \mathrm{E}-02$ & $2.32 \mathrm{E}-06$ \\
\hline TOTAL & $\mathbf{6 . 4 7 E}+\mathbf{0 0}$ & $\mathbf{4 . 2 2 E - 0 1}$ & $7.60 \mathrm{E}-02$ & $\mathbf{2 . 3 6 E}+\mathbf{0 0}$ & $\mathbf{8 . 2 8 E}-02$ & $4.41 \mathrm{E}-04$ \\
\hline
\end{tabular}

Filter

\begin{tabular}{|c|c|c|c|c|c|c|}
\hline \multirow{3}{*}{ Isotope } & \multicolumn{6}{|c|}{ Dose rates, $\mathrm{mSv} / \mathrm{h}$} \\
\hline & \multicolumn{3}{|c|}{ Horizontal Axis $50 \mathrm{~cm}$} & \multicolumn{3}{|c|}{ Vertical Axis $50 \mathrm{~cm}$} \\
\hline & Shutdown & 2 days & 10 days & Shutdown & 2 days & 10 days \\
\hline $\mathrm{Fe}-59$ & $2.16 \mathrm{E}-03$ & $2.09 \mathrm{E}-03$ & $1.85 \mathrm{E}-03$ & $2.26 \mathrm{E}-03$ & $2.19 \mathrm{E}-03$ & $1.93 \mathrm{E}-03$ \\
\hline $\mathrm{Mn}-54$ & $9.79 \mathrm{E}-03$ & $9.75 \mathrm{E}-03$ & $9.58 \mathrm{E}-03$ & $1.06 \mathrm{E}-02$ & $1.05 \mathrm{E}-02$ & $1.03 \mathrm{E}-02$ \\
\hline $\mathrm{Fe}-55$ & $1.58 \mathrm{E}-13$ & $1.58 \mathrm{E}-13$ & $1.57 \mathrm{E}-13$ & $2.19 \mathrm{E}-13$ & $2.19 \mathrm{E}-13$ & $2.18 \mathrm{E}-13$ \\
\hline Co-58 & $1.71 \mathrm{E}-02$ & $1.68 \mathrm{E}-02$ & $1.55 \mathrm{E}-02$ & $1.86 \mathrm{E}-02$ & $1.83 \mathrm{E}-02$ & $1.69 \mathrm{E}-02$ \\
\hline Co-60 & $2.57 \mathrm{E}-02$ & $2.57 \mathrm{E}-02$ & $2.56 \mathrm{E}-02$ & $2.68 \mathrm{E}-02$ & $2.67 \mathrm{E}-02$ & $2.67 \mathrm{E}-02$ \\
\hline $\mathrm{Cr}-51$ & $1.41 \mathrm{E}-03$ & $1.34 \mathrm{E}-03$ & $1.10 \mathrm{E}-03$ & $1.66 \mathrm{E}-03$ & $1.58 \mathrm{E}-03$ & $1.29 \mathrm{E}-03$ \\
\hline $\mathrm{Mn}-56$ & $1.05 \mathrm{E}+00$ & $2.60 \mathrm{E}-06$ & $9.88 \mathrm{E}-29$ & $1.09 \mathrm{E}+00$ & $2.70 \mathrm{E}-06$ & $1.03 \mathrm{E}-28$ \\
\hline $\mathrm{Cu}-64$ & $4.09 \mathrm{E}+00$ & $2.98 \mathrm{E}-01$ & $8.39 \mathrm{E}-06$ & $4.61 \mathrm{E}+00$ & $3.36 \mathrm{E}-01$ & $9.45 \mathrm{E}-06$ \\
\hline TOTAL & $5.19 \mathrm{E}+00$ & $3.54 \mathrm{E}-01$ & 5.37E-02 & $5.76 \mathrm{E}+00$ & 3.95E-01 & $5.71 \mathrm{E}-02$ \\
\hline
\end{tabular}


Table 12. Dose rates $(\mathrm{mSv} / \mathrm{h})$ for individual isotopes at $100 \mathrm{~cm}^{3}$ from the equipment outer surface at shutdown, 2 days, and 10 days after shutdown

Ion Exchanger

\begin{tabular}{|l|c|c|c|c|c|c|}
\hline \multirow{3}{*}{ Isotope } & \multicolumn{6}{|c|}{ Dose rates, mSv/h } \\
\cline { 2 - 7 } & \multicolumn{2}{|c|}{ Horizontal Axis 100 cm } & \multicolumn{3}{c|}{ Vertical Axis 75 cm } \\
\cline { 2 - 7 } & Shutdown & 2 days & 10 days & Shutdown & 2 days & 10 days \\
\hline Fe-59 & $1.81 \mathrm{E}-03$ & $1.75 \mathrm{E}-03$ & $1.55 \mathrm{E}-03$ & $2.85 \mathrm{E}-05$ & $2.76 \mathrm{E}-05$ & $2.44 \mathrm{E}-05$ \\
\hline Mn-54 & $6.98 \mathrm{E}-03$ & $6.95 \mathrm{E}-03$ & $6.82 \mathrm{E}-03$ & $4.39 \mathrm{E}-05$ & $4.37 \mathrm{E}-05$ & $4.30 \mathrm{E}-05$ \\
\hline Fe-55 & $8.46 \mathrm{E}-12$ & $8.45 \mathrm{E}-12$ & $8.40 \mathrm{E}-12$ & $4.06 \mathrm{E}-12$ & $4.06 \mathrm{E}-12$ & $4.04 \mathrm{E}-12$ \\
\hline Co-58 & $1.17 \mathrm{E}-02$ & $1.15 \mathrm{E}-02$ & $1.06 \mathrm{E}-02$ & $9.90 \mathrm{E}-05$ & $9.71 \mathrm{E}-05$ & $8.98 \mathrm{E}-05$ \\
\hline Co-60 & $2.02 \mathrm{E}-02$ & $2.02 \mathrm{E}-02$ & $2.01 \mathrm{E}-02$ & $2.29 \mathrm{E}-04$ & $2.29 \mathrm{E}-04$ & $2.29 \mathrm{E}-04$ \\
\hline Cr-51 & $6.39 \mathrm{E}-04$ & $6.08 \mathrm{E}-04$ & $4.98 \mathrm{E}-04$ & $1.24 \mathrm{E}-06$ & $1.18 \mathrm{E}-06$ & $9.69 \mathrm{E}-07$ \\
\hline Mn-56 & $9.24 \mathrm{E}-01$ & $2.30 \mathrm{E}-06$ & $8.73 \mathrm{E}-29$ & $1.01 \mathrm{E}+00$ & $2.51 \mathrm{E}-06$ & $9.55 \mathrm{E}-29$ \\
\hline Cu-64 & $2.52 \mathrm{E}+00$ & $1.83 \mathrm{E}-01$ & $5.16 \mathrm{E}-06$ & $9.30 \mathrm{E}-01$ & $6.78 \mathrm{E}-02$ & $1.91 \mathrm{E}-06$ \\
\hline TOTAL & $\mathbf{3 . 4 8 E}+\mathbf{0 0}$ & $\mathbf{2 . 2 4 E - 0 1}$ & $\mathbf{3 . 9 6 E - 0 2}$ & $\mathbf{1 . 9 4 E}+\mathbf{0 0}$ & $\mathbf{6 . 8 2 E}-02$ & $3.89 \mathrm{E}-04$ \\
\hline
\end{tabular}

Filter

\begin{tabular}{|l|c|c|c|c|c|c|}
\hline \multirow{3}{*}{ Isotope } & \multicolumn{6}{|c|}{ Dose rates, $\mathbf{~ m S v / h}$} \\
\cline { 2 - 7 } & \multicolumn{2}{|c|}{ Horizontal Axis 100 cm } & \multicolumn{3}{c|}{ Vertical Axis 100 cm } \\
\cline { 2 - 7 } & Shutdown & 2 days & 10 days & Shutdown & 2 days & 10 days \\
\hline Fe-59 & $9.30 \mathrm{E}-04$ & $9.01 \mathrm{E}-04$ & $7.96 \mathrm{E}-04$ & $8.11 \mathrm{E}-04$ & $7.86 \mathrm{E}-04$ & $6.94 \mathrm{E}-04$ \\
\hline Mn-54 & $4.24 \mathrm{E}-03$ & $4.22 \mathrm{E}-03$ & $4.15 \mathrm{E}-03$ & $3.79 \mathrm{E}-03$ & $3.78 \mathrm{E}-03$ & $3.71 \mathrm{E}-03$ \\
\hline Fe-55 & $7.35 \mathrm{E}-14$ & $7.34 \mathrm{E}-14$ & $7.30 \mathrm{E}-14$ & $8.38 \mathrm{E}-14$ & $8.36 \mathrm{E}-14$ & $8.32 \mathrm{E}-14$ \\
\hline Co-58 & $7.44 \mathrm{E}-03$ & $7.30 \mathrm{E}-03$ & $6.75 \mathrm{E}-03$ & $6.70 \mathrm{E}-03$ & $6.57 \mathrm{E}-03$ & $6.08 \mathrm{E}-03$ \\
\hline Co-60 & $1.11 \mathrm{E}-02$ & $1.11 \mathrm{E}-02$ & $1.10 \mathrm{E}-02$ & $9.65 \mathrm{E}-03$ & $9.64 \mathrm{E}-03$ & $9.62 \mathrm{E}-03$ \\
\hline Cr-51 & $6.23 \mathrm{E}-04$ & $5.93 \mathrm{E}-04$ & $4.85 \mathrm{E}-04$ & $6.00 \mathrm{E}-04$ & $5.71 \mathrm{E}-04$ & $4.67 \mathrm{E}-04$ \\
\hline Mn-56 & $4.52 \mathrm{E}-01$ & $1.12 \mathrm{E}-06$ & $4.27 \mathrm{E}-29$ & $3.91 \mathrm{E}-01$ & $9.71 \mathrm{E}-07$ & $3.70 \mathrm{E}-29$ \\
\hline Cu-64 & $1.79 \mathrm{E}+00$ & $1.30 \mathrm{E}-01$ & $3.66 \mathrm{E}-06$ & $1.65 \mathrm{E}+00$ & $1.20 \mathrm{E}-01$ & $3.38 \mathrm{E}-06$ \\
\hline TOTAL & $\mathbf{2 . 2 6 E}+\mathbf{0 0}$ & $\mathbf{1 . 5 4 E - 0 1}$ & $\mathbf{2 . 3 2 E - 0 2}$ & $\mathbf{2 . 0 6 E}+00$ & $\mathbf{1 . 4 1 E}-01$ & $2.06 \mathrm{E}-02$ \\
\hline
\end{tabular}

\footnotetext{
${ }^{3}$ Distance on the vertical axis above the ion exchanger is limited to $75 \mathrm{~cm}$.
} 
Table 13. Summary of dose rates $(\mathrm{mSv} / \mathrm{h})$ at $5 \mathrm{~cm}, 30 \mathrm{~cm}, 50 \mathrm{~cm}$, and $100 \mathrm{~cm}^{4}$ from the equipment outer surface at shutdown, 2 days, and 10 days after shutdown

Ion Exchanger

\begin{tabular}{|l|c|c|c|c|c|c|}
\hline \multirow{3}{*}{$\begin{array}{c}\text { Distance } \\
(\mathbf{c m})\end{array}$} & \multicolumn{3}{|c|}{ Dorizontal Axis } & & \multicolumn{3}{c|}{ Vertical Axis } \\
\cline { 2 - 7 } & Shutdown & 2 days & 10 days & Shutdown & 2 days & 10 days \\
\cline { 2 - 7 } & $1.21 \mathrm{E}+01$ & $7.92 \mathrm{E}-01$ & $1.49 \mathrm{E}-01$ & $2.88 \mathrm{E}+00$ & $9.99 \mathrm{E}-02$ & $5.55 \mathrm{E}-04$ \\
\hline 30 & $8.52 \mathrm{E}+00$ & $5.57 \mathrm{E}-01$ & $1.02 \mathrm{E}-01$ & $2.64 \mathrm{E}+00$ & $9.14 \mathrm{E}-02$ & $5.16 \mathrm{E}-04$ \\
\hline 50 & $6.47 \mathrm{E}+00$ & $4.22 \mathrm{E}-01$ & $7.60 \mathrm{E}-02$ & $2.36 \mathrm{E}+00$ & $8.28 \mathrm{E}-02$ & $4.41 \mathrm{E}-04$ \\
\hline $100(75)$ & $3.48 \mathrm{E}+00$ & $2.24 \mathrm{E}-01$ & $3.96 \mathrm{E}-02$ & $1.94 \mathrm{E}+00$ & $6.82 \mathrm{E}-02$ & $3.89 \mathrm{E}-04$ \\
\hline
\end{tabular}

Filter

\begin{tabular}{|l|c|c|c|c|c|c|}
\hline \multirow{2}{*}{$\begin{array}{c}\text { Distance } \\
(\mathbf{c m})\end{array}$} & \multicolumn{4}{|c|}{ Dorizontal Axis } & \multicolumn{3}{c|}{ Vertical Axis } \\
\cline { 2 - 7 } & Shutdown & 2 days & 10 days & Shutdown & 2 days & 10 days \\
\cline { 2 - 7 } 5 & $1.50 \mathrm{E}+01$ & $1.01 \mathrm{E}+00$ & $1.58 \mathrm{E}-01$ & $1.84 \mathrm{E}+01$ & $1.25 \mathrm{E}+00$ & $1.93 \mathrm{E}-01$ \\
\hline 30 & $7.91 \mathrm{E}+00$ & $5.38 \mathrm{E}-01$ & $8.22 \mathrm{E}-02$ & $9.87 \mathrm{E}+00$ & $6.77 \mathrm{E}-01$ & $9.88 \mathrm{E}-02$ \\
\hline 50 & $5.19 \mathrm{E}+00$ & $3.54 \mathrm{E}-01$ & $5.37 \mathrm{E}-02$ & $5.76 \mathrm{E}+00$ & $3.95 \mathrm{E}-01$ & $5.71 \mathrm{E}-02$ \\
\hline 100 & $2.26 \mathrm{E}+00$ & $1.54 \mathrm{E}-01$ & $2.32 \mathrm{E}-02$ & $2.06 \mathrm{E}+00$ & $1.41 \mathrm{E}-01$ & $2.06 \mathrm{E}-02$ \\
\hline
\end{tabular}

\footnotetext{
${ }^{4}$ Distance on the vertical axis above the ion exchanger is limited to $75 \mathrm{~cm}$.
} 
Table 14. Dose rates $(\mathrm{mSv} / \mathrm{h})$ at shutdown for individual isotopes (distance $X$ and $Z$ is from the equipment outer surface)

Ion Exchanger

\begin{tabular}{|c|c|c|c|c|c|c|c|c|}
\hline \multirow{3}{*}{ Isotope } & \multicolumn{8}{|c|}{ Dose rates, $\mathrm{mSv} / \mathrm{h}$} \\
\hline & \multicolumn{4}{|c|}{ Horizontal Axis $X, \mathrm{~cm}$} & \multicolumn{4}{|c|}{ Vertical Axis X, cm } \\
\hline & 5 & 30 & 50 & 100 & 5 & 30 & 50 & 75 \\
\hline $\mathrm{Fe}-59$ & $6.88 \mathrm{E}-03$ & $4.71 \mathrm{E}-03$ & $3.48 \mathrm{E}-03$ & $1.81 \mathrm{E}-03$ & 4.22E-05 & $3.92 \mathrm{E}-05$ & $3.39 \mathrm{E}-05$ & $2.85 \mathrm{E}-05$ \\
\hline Mn-54 & $2.58 \mathrm{E}-02$ & $1.79 \mathrm{E}-02$ & $1.33 \mathrm{E}-02$ & $6.98 \mathrm{E}-03$ & $5.99 \mathrm{E}-05$ & $5.70 \mathrm{E}-05$ & 4.79E-05 & 4.39E-05 \\
\hline Fe-55 & $6.52 \mathrm{E}-12$ & $6.95 \mathrm{E}-12$ & $7.38 \mathrm{E}-12$ & $8.46 \mathrm{E}-12$ & $3.06 \mathrm{E}-12$ & $4.18 \mathrm{E}-12$ & $4.11 \mathrm{E}-12$ & $4.06 \mathrm{E}-12$ \\
\hline Co-58 & $4.29 \mathrm{E}-02$ & $2.98 \mathrm{E}-02$ & $2.23 \mathrm{E}-02$ & $1.17 \mathrm{E}-02$ & $1.35 \mathrm{E}-04$ & $1.31 \mathrm{E}-04$ & $1.08 \mathrm{E}-04$ & $9.90 \mathrm{E}-05$ \\
\hline Co-60 & $7.73 \mathrm{E}-02$ & 5.29E-02 & $3.90 \mathrm{E}-02$ & $2.02 \mathrm{E}-02$ & $3.35 \mathrm{E}-04$ & $3.05 \mathrm{E}-04$ & $2.65 \mathrm{E}-04$ & $2.29 \mathrm{E}-04$ \\
\hline $\mathrm{Cr}-51$ & $2.19 \mathrm{E}-03$ & $1.57 \mathrm{E}-03$ & $1.20 \mathrm{E}-03$ & $6.39 \mathrm{E}-04$ & $1.28 \mathrm{E}-06$ & $1.31 \mathrm{E}-06$ & $1.18 \mathrm{E}-06$ & $1.24 \mathrm{E}-06$ \\
\hline Mn-56 & $3.19 \mathrm{E}+00$ & $2.23 \mathrm{E}+00$ & $1.68 \mathrm{E}+00$ & $9.24 \mathrm{E}-01$ & $1.52 \mathrm{E}+00$ & $1.39 \mathrm{E}+00$ & $1.23 \mathrm{E}+00$ & $1.01 \mathrm{E}+00$ \\
\hline $\mathrm{Cu}-64$ & $8.76 \mathrm{E}+00$ & $6.19 \mathrm{E}+00$ & $4.71 \mathrm{E}+00$ & $2.52 \mathrm{E}+00$ & $1.36 \mathrm{E}+00$ & $1.25 \mathrm{E}+00$ & $1.13 \mathrm{E}+00$ & $9.30 \mathrm{E}-01$ \\
\hline TOTAL & $1.21 \mathrm{E}+01$ & $8.52 \mathrm{E}+00$ & $6.47 \mathrm{E}+00$ & $3.48 \mathrm{E}+00$ & $2.88 \mathrm{E}+00$ & $2.64 \mathrm{E}+00$ & $2.36 \mathrm{E}+00$ & $1.94 \mathrm{E}+00$ \\
\hline
\end{tabular}

Filter

\begin{tabular}{|c|c|c|c|c|c|c|c|c|}
\hline \multirow{3}{*}{ Isotope } & \multicolumn{8}{|c|}{ Dose rates, $\mathrm{mSv} / \mathrm{h}$} \\
\hline & \multicolumn{4}{|c|}{ Horizontal Axis X, cm } & \multicolumn{4}{|c|}{ Vertical Axis X, cm } \\
\hline & 5 & 30 & 50 & 100 & 5 & 30 & 50 & 100 \\
\hline Fe-59 & $6.41 \mathrm{E}-03$ & $3.31 \mathrm{E}-03$ & $2.16 \mathrm{E}-03$ & $9.30 \mathrm{E}-04$ & $7.80 \mathrm{E}-03$ & $3.91 \mathrm{E}-03$ & $2.26 \mathrm{E}-03$ & $8.11 \mathrm{E}-04$ \\
\hline $\mathrm{Mn}-54$ & $2.86 \mathrm{E}-02$ & $1.50 \mathrm{E}-02$ & $9.79 \mathrm{E}-03$ & $4.24 \mathrm{E}-03$ & $3.51 \mathrm{E}-02$ & $1.83 \mathrm{E}-02$ & $1.06 \mathrm{E}-02$ & $3.79 \mathrm{E}-03$ \\
\hline $\mathrm{Fe}-55$ & $3.88 \mathrm{E}-13$ & $2.26 \mathrm{E}-13$ & $1.58 \mathrm{E}-13$ & $7.35 \mathrm{E}-14$ & $4.62 \mathrm{E}-13$ & $3.38 \mathrm{E}-13$ & $2.19 \mathrm{E}-13$ & $8.38 \mathrm{E}-14$ \\
\hline $\mathrm{Co}-58$ & 4.97E-02 & $2.61 \mathrm{E}-02$ & $1.71 \mathrm{E}-02$ & $7.44 \mathrm{E}-03$ & $6.11 \mathrm{E}-02$ & $3.21 \mathrm{E}-02$ & $1.86 \mathrm{E}-02$ & $6.70 \mathrm{E}-03$ \\
\hline Co-60 & $7.68 \mathrm{E}-02$ & $3.96 \mathrm{E}-02$ & $2.57 \mathrm{E}-02$ & $1.11 \mathrm{E}-02$ & $9.33 \mathrm{E}-02$ & $4.64 \mathrm{E}-02$ & $2.68 \mathrm{E}-02$ & $9.65 \mathrm{E}-03$ \\
\hline Cr-51 & $3.89 \mathrm{E}-03$ & $2.11 \mathrm{E}-03$ & $1.41 \mathrm{E}-03$ & $6.23 \mathrm{E}-04$ & $4.80 \mathrm{E}-03$ & $2.80 \mathrm{E}-03$ & $1.66 \mathrm{E}-03$ & $6.00 \mathrm{E}-04$ \\
\hline $\mathrm{Mn}-56$ & $3.14 \mathrm{E}+00$ & $1.61 \mathrm{E}+00$ & $1.05 \mathrm{E}+00$ & $4.52 \mathrm{E}-01$ & $3.79 \mathrm{E}+00$ & $1.88 \mathrm{E}+00$ & $1.09 \mathrm{E}+00$ & $3.91 \mathrm{E}-01$ \\
\hline $\mathrm{Cu}-64$ & $1.17 \mathrm{E}+01$ & $6.22 \mathrm{E}+00$ & $4.09 \mathrm{E}+00$ & $1.79 \mathrm{E}+00$ & $1.44 \mathrm{E}+01$ & $7.88 \mathrm{E}+00$ & $4.61 \mathrm{E}+00$ & $1.65 \mathrm{E}+00$ \\
\hline TOTAL & $1.50 \mathrm{E}+01$ & $7.91 \mathrm{E}+00$ & $5.19 \mathrm{E}+00$ & $2.26 \mathrm{E}+00$ & $1.84 \mathrm{E}+01$ & $9.87 \mathrm{E}+00$ & $5.76 \mathrm{E}+00$ & $2.06 \mathrm{E}+00$ \\
\hline
\end{tabular}


Table 15. Conversion Factors (mSv/h-per-GBq) for individual isotopes (distance $\mathrm{X}$ and $\mathrm{Z}$ is given from the equipment outer surface)

Ion Exchanger

\begin{tabular}{|c|c|c|c|c|c|c|c|c|}
\hline \multirow{3}{*}{ Isotope } & \multicolumn{8}{|c|}{ Conversion Factor, mSv/h per GBq } \\
\hline & \multicolumn{4}{|c|}{ Horizontal Axis $X, \mathrm{~cm}$} & \multicolumn{4}{|c|}{ Vertical Axis $X, \mathrm{~cm}$} \\
\hline & 5 & 30 & 50 & 100 & 5 & 30 & 50 & 75 \\
\hline Fe-59 & $1.27 \mathrm{E}-02$ & $8.71 \mathrm{E}-03$ & $6.43 \mathrm{E}-03$ & $3.34 \mathrm{E}-03$ & $7.81 \mathrm{E}-05$ & $7.25 \mathrm{E}-05$ & $6.26 \mathrm{E}-05$ & $5.26 \mathrm{E}-05$ \\
\hline $\mathrm{Mn}-54$ & $7.52 \mathrm{E}-03$ & $5.20 \mathrm{E}-03$ & $3.88 \mathrm{E}-03$ & $2.03 \mathrm{E}-03$ & $1.75 \mathrm{E}-05$ & $1.66 \mathrm{E}-05$ & $1.40 \mathrm{E}-05$ & $1.28 \mathrm{E}-05$ \\
\hline $\mathrm{Fe}-55$ & $2.20 \mathrm{E}-12$ & $2.35 \mathrm{E}-12$ & $2.49 \mathrm{E}-12$ & $2.86 \mathrm{E}-12$ & $1.03 \mathrm{E}-12$ & $1.41 \mathrm{E}-12$ & $1.39 \mathrm{E}-12$ & $1.37 \mathrm{E}-12$ \\
\hline Co-58 & $8.27 \mathrm{E}-03$ & $5.75 \mathrm{E}-03$ & $4.30 \mathrm{E}-03$ & $2.25 \mathrm{E}-03$ & $2.61 \mathrm{E}-05$ & $2.52 \mathrm{E}-05$ & $2.09 \mathrm{E}-05$ & $1.91 \mathrm{E}-05$ \\
\hline Co-60 & $2.77 \mathrm{E}-02$ & $1.89 \mathrm{E}-02$ & $1.40 \mathrm{E}-02$ & $7.23 \mathrm{E}-03$ & $1.20 \mathrm{E}-04$ & $1.09 \mathrm{E}-04$ & $9.49 \mathrm{E}-05$ & $8.22 \mathrm{E}-05$ \\
\hline Cr-51 & $1.23 \mathrm{E}-04$ & $8.82 \mathrm{E}-05$ & $6.73 \mathrm{E}-05$ & $3.58 \mathrm{E}-05$ & $7.15 \mathrm{E}-08$ & $7.36 \mathrm{E}-08$ & $6.61 \mathrm{E}-08$ & $6.97 \mathrm{E}-08$ \\
\hline Mn-56 & $1.50 \mathrm{E}-02$ & $1.05 \mathrm{E}-02$ & $7.88 \mathrm{E}-03$ & $4.34 \mathrm{E}-03$ & $7.14 \mathrm{E}-03$ & $6.54 \mathrm{E}-03$ & $5.76 \mathrm{E}-03$ & $4.74 \mathrm{E}-03$ \\
\hline $\mathrm{Cu}-64$ & $1.14 \mathrm{E}-03$ & 8.07E-04 & $6.14 \mathrm{E}-04$ & $3.28 \mathrm{E}-04$ & $1.78 \mathrm{E}-04$ & $1.63 \mathrm{E}-04$ & $1.47 \mathrm{E}-04$ & $1.21 \mathrm{E}-04$ \\
\hline
\end{tabular}

Filter

\begin{tabular}{|c|c|c|c|c|c|c|c|c|}
\hline \multirow{3}{*}{ Isotope } & \multicolumn{8}{|c|}{ Conversion Factor, mSv/h per GBq } \\
\hline & \multicolumn{4}{|c|}{ Horizontal Axis X, cm } & \multicolumn{4}{|c|}{ Vertical Axis X, cm } \\
\hline & 5 & 30 & 50 & 100 & 5 & 30 & 50 & 100 \\
\hline Fe-59 & $1.28 \mathrm{E}-01$ & $6.63 \mathrm{E}-02$ & $4.31 \mathrm{E}-02$ & $1.86 \mathrm{E}-02$ & $1.56 \mathrm{E}-01$ & $7.81 \mathrm{E}-02$ & $4.51 \mathrm{E}-02$ & $1.62 \mathrm{E}-02$ \\
\hline $\mathrm{Mn}-54$ & $8.42 \mathrm{E}-02$ & $4.40 \mathrm{E}-02$ & $2.88 \mathrm{E}-02$ & $1.25 \mathrm{E}-02$ & $1.03 \mathrm{E}-01$ & $5.37 \mathrm{E}-02$ & $3.11 \mathrm{E}-02$ & $1.12 \mathrm{E}-02$ \\
\hline $\mathrm{Fe}-55$ & $2.42 \mathrm{E}-12$ & $1.41 \mathrm{E}-12$ & $9.86 \mathrm{E}-13$ & $4.59 \mathrm{E}-13$ & $2.89 \mathrm{E}-12$ & $2.11 \mathrm{E}-12$ & $1.37 \mathrm{E}-12$ & $5.23 \mathrm{E}-13$ \\
\hline $\mathrm{Co}-58$ & $9.56 \mathrm{E}-02$ & $5.02 \mathrm{E}-02$ & $3.29 \mathrm{E}-02$ & $1.43 \mathrm{E}-02$ & $1.18 \mathrm{E}-01$ & $6.18 \mathrm{E}-02$ & $3.58 \mathrm{E}-02$ & $1.29 \mathrm{E}-02$ \\
\hline Co-60 & $2.74 \mathrm{E}-01$ & $1.41 \mathrm{E}-01$ & $9.18 \mathrm{E}-02$ & $3.96 \mathrm{E}-02$ & $3.33 \mathrm{E}-01$ & $1.66 \mathrm{E}-01$ & $9.56 \mathrm{E}-02$ & $3.45 \mathrm{E}-02$ \\
\hline Cr-51 & $2.18 \mathrm{E}-03$ & $1.18 \mathrm{E}-03$ & $7.91 \mathrm{E}-04$ & $3.50 \mathrm{E}-04$ & $2.70 \mathrm{E}-03$ & $1.57 \mathrm{E}-03$ & $9.31 \mathrm{E}-04$ & $3.37 \mathrm{E}-04$ \\
\hline Mn-56 & $1.86 \mathrm{E}-01$ & $9.51 \mathrm{E}-02$ & $6.17 \mathrm{E}-02$ & $2.67 \mathrm{E}-02$ & $2.24 \mathrm{E}-01$ & $1.11 \mathrm{E}-01$ & $6.42 \mathrm{E}-02$ & $2.31 \mathrm{E}-02$ \\
\hline $\mathrm{Cu}-64$ & $1.65 \mathrm{E}-02$ & $8.78 \mathrm{E}-03$ & $5.78 \mathrm{E}-03$ & $2.52 \mathrm{E}-03$ & $2.03 \mathrm{E}-02$ & $1.11 \mathrm{E}-02$ & $6.51 \mathrm{E}-03$ & $2.33 \mathrm{E}-03$ \\
\hline
\end{tabular}




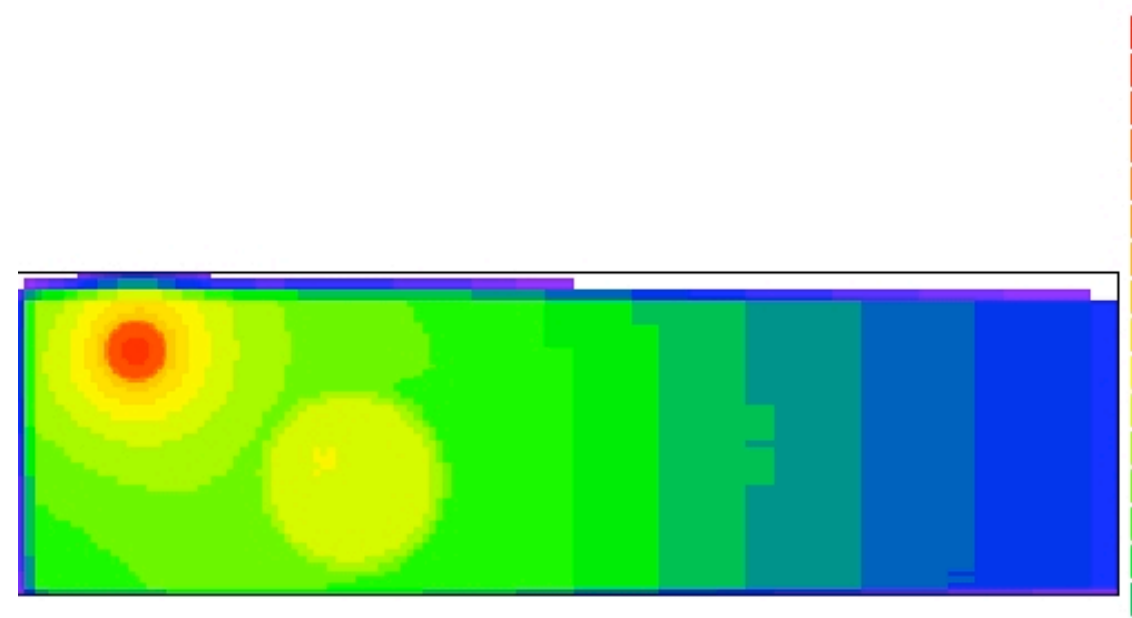

photon Results

Total photon Results

Values

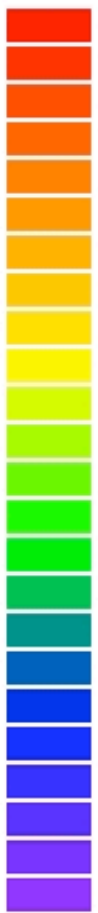

$6.81 \mathrm{E} 01-1.00 \mathrm{E} 02$

$4.64 \mathrm{EO}-6.81 \mathrm{E01}$

$3.16 \mathrm{E} 01-4.64 \mathrm{E} 01$

$2.15 \mathrm{E} 01-3.16 \mathrm{E} 01$

$1.47 E 01-2.15 E 01$

$1.00 \mathrm{E} 01-1.47 \mathrm{E} 01$

$6.81 \mathrm{E} 00-1.00 \mathrm{E} 01$

$4.64 \mathrm{E} 00-6.81 \mathrm{E} 00$

$3.16 \mathrm{E} 00-4.64 \mathrm{E} 00$

$2.15 \mathrm{E} 00-3.16 \mathrm{E} 00$

$1.47 \mathrm{E} 00-2.15 \mathrm{E} 00$

$1.00 \mathrm{E} 00-1.47 \mathrm{E} 00$

$6.81 \mathrm{E}-01-1.00 \mathrm{E} 00$

4.64E-01 - 6.81E-01

$3.16 \mathrm{E}-01-4.64 \mathrm{E}-01$

2.15E-01 - 3.16E-01

$1.47 \mathrm{E}-01-2.15 \mathrm{E}-01$

$1.00 \mathrm{E}-01-1.47 \mathrm{E}-01$

$6.81 \mathrm{E}-02-1.00 \mathrm{E}-01$

$4.64 \mathrm{E}-02-6.81 \mathrm{E}-02$

$3.16 \mathrm{E}-02-4.64 \mathrm{E}-02$

$2.15 \mathrm{E}-02-3.16 \mathrm{E}-02$

$1.47 \mathrm{E}-02-2.15 \mathrm{E}-02$

$1.00 \mathrm{E}-02-1.47 \mathrm{E}-02$
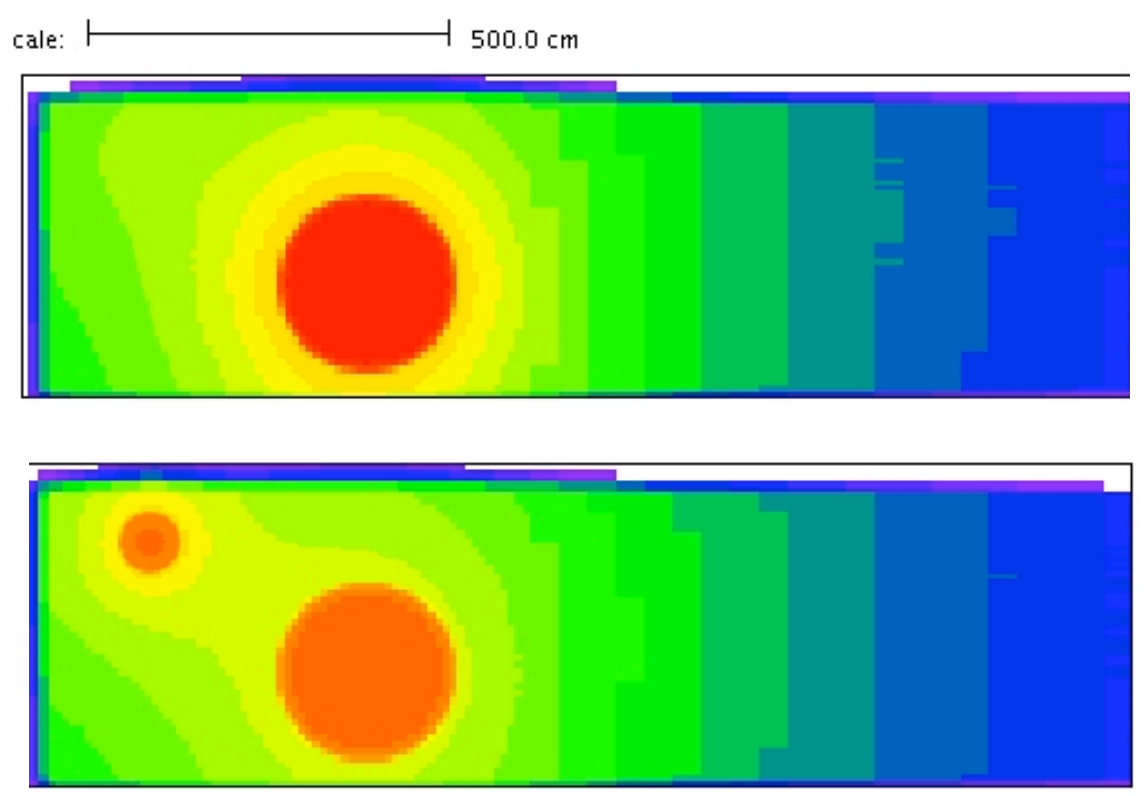

Fig. 4. XY-plane mesh tally dose rate $(\mathrm{mSv} / \mathrm{h})$ contour plots through vertical center of filter (top), ion exchanger resin (center), and ion exchanger water (bottom) for standard concrete walls/ceiling at shutdown. Distance scale and color-coded dose rate values are the same for all three plots. 


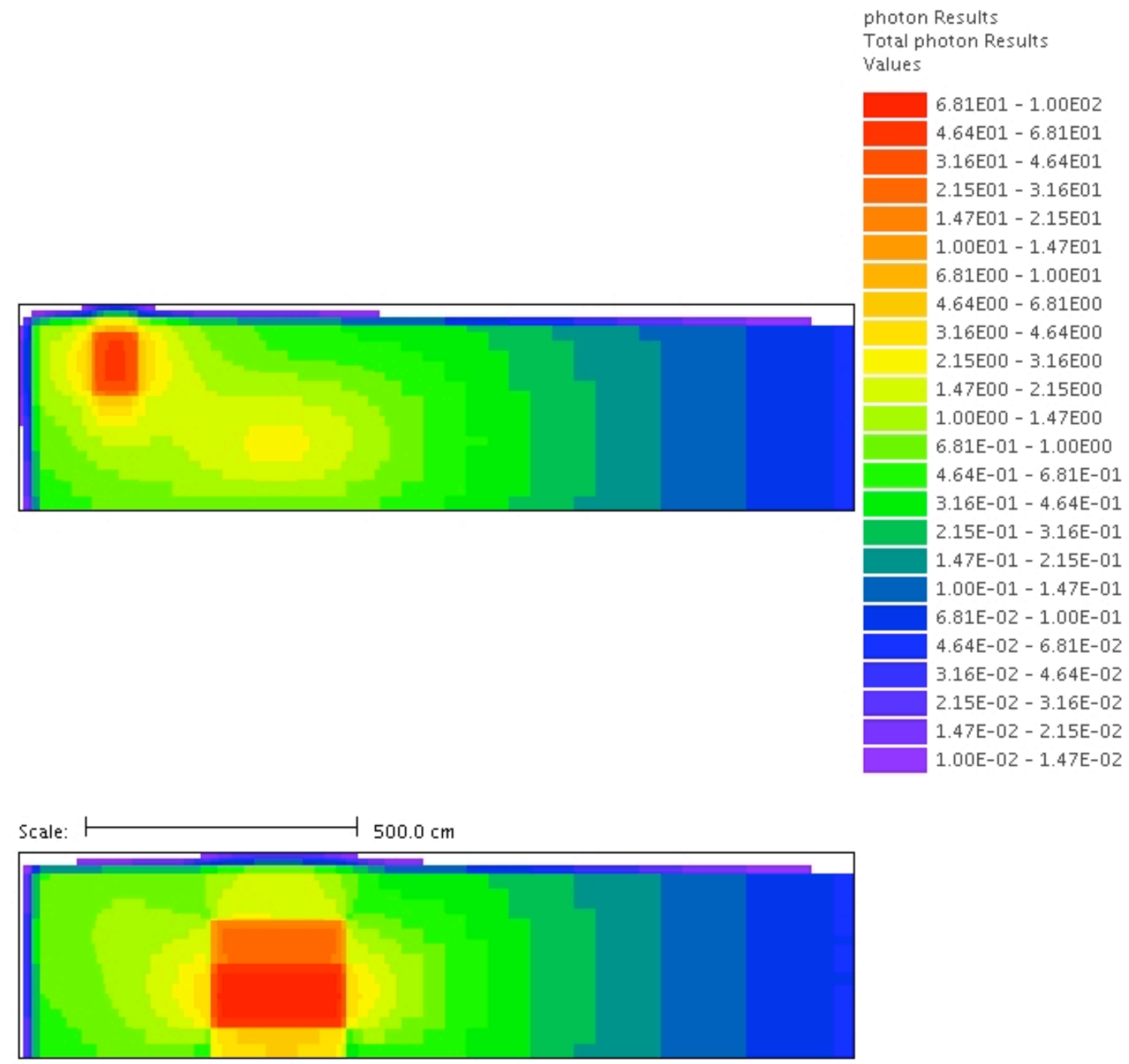

Fig. 5. XZ-plane mesh tally dose rate $(\mathrm{mSv} / \mathrm{h})$ contour plots through $\mathrm{Y}$-axis center of filter (top) and ion exchanger (bottom) for standard concrete walls/ceiling at shutdown. Distance scale and color-coded dose rate values are the same for both plots. 


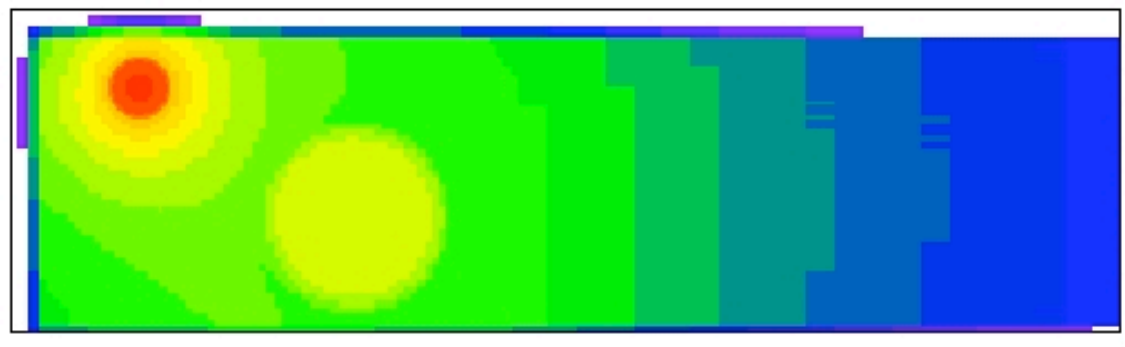

photon Results

Total photon Results

Values

\begin{tabular}{|l|l}
\hline $6.81 E 01-1.00 E 02$ \\
\hline $4.64 E 01-6.81 E 01$ \\
$3.16 E 01-4.64 \mathrm{E} 01$ \\
\hline $2.15 \mathrm{E} 01-3.16 \mathrm{E} 01$ \\
$1.47 \mathrm{E} 01-2.15 \mathrm{E} 01$ \\
$1.00 \mathrm{E} 01-1.47 \mathrm{E} 01$ \\
$6.81 \mathrm{E} 00-1.00 \mathrm{E} 01$ \\
\hline $4.64 \mathrm{E} 00-6.81 \mathrm{E} 00$ \\
\hline $3.16 \mathrm{E} 00-4.64 \mathrm{E} 00$ \\
\hline $2.15 \mathrm{E} 00-3.16 \mathrm{E} 00$ \\
\hline $1.47 \mathrm{E} 00-2.15 \mathrm{E} 00$ \\
\hline $1.00 \mathrm{E} 00-1.47 \mathrm{E} 00$ \\
\hline $6.81 \mathrm{E}-01-1.00 \mathrm{E} 00$ \\
\hline $4.64 \mathrm{E}-01-6.81 \mathrm{E}-01$ \\
\hline $3.16 \mathrm{E}-01-4.64 \mathrm{E}-01$ \\
\hline $2.15 \mathrm{E}-01-3.16 \mathrm{E}-01$ \\
\hline $1.47 \mathrm{E}-01-2.15 \mathrm{E}-01$ \\
\hline $1.00 \mathrm{E}-01-1.47 \mathrm{E}-01$ \\
\hline $6.81 \mathrm{E}-02-1.00 \mathrm{E}-01$ \\
\hline $4.64 \mathrm{E}-02-6.81 \mathrm{E}-02$ \\
\hline $3.16 \mathrm{E}-02-4.64 \mathrm{E}-02$ \\
\hline $2.15 \mathrm{E}-02-3.16 \mathrm{E}-02$ \\
\hline $1.47 \mathrm{E}-02-2.15 \mathrm{E}-02$ \\
\hline $1.00 \mathrm{E}-02-1.47 \mathrm{E}-02$ \\
\hline
\end{tabular}

Scale: $\longmapsto 500.0 \mathrm{~cm}$
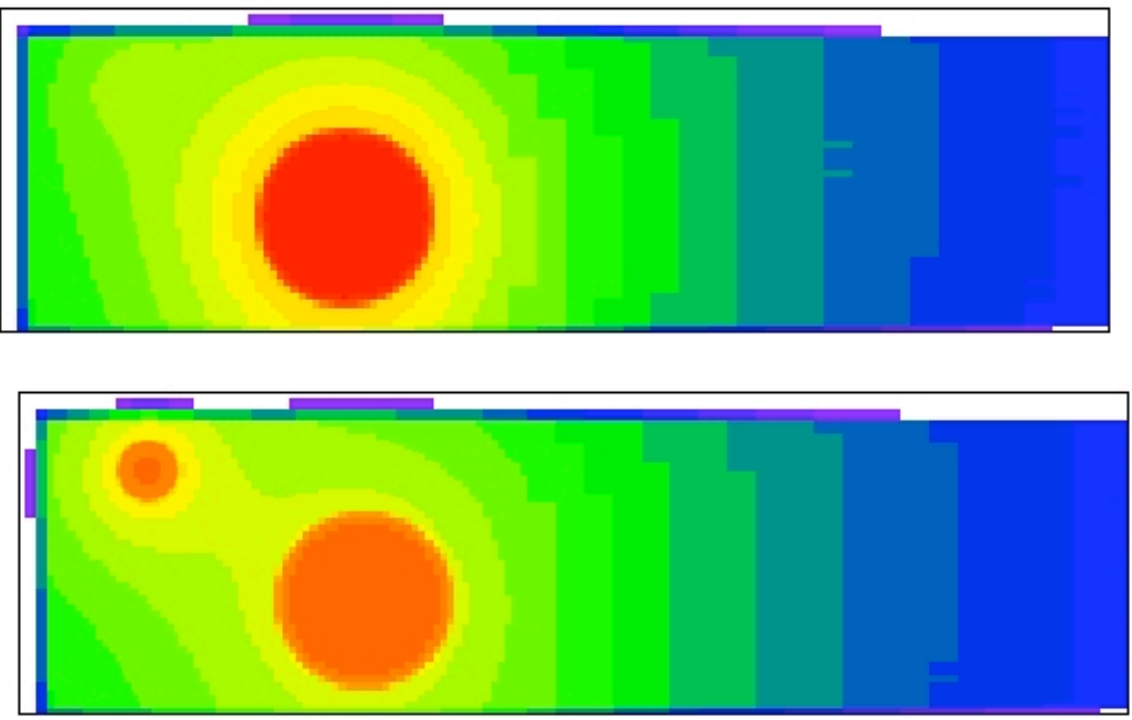

Fig. 6. XY-plane mesh tally dose rate (mSv/h) contour plots through vertical center of filter (top), ion exchanger resin (center), and ion exchanger water (bottom) for barytes concrete walls/ceiling at shutdown. Distance scale and color-coded dose rate values are the same for all three plots. 


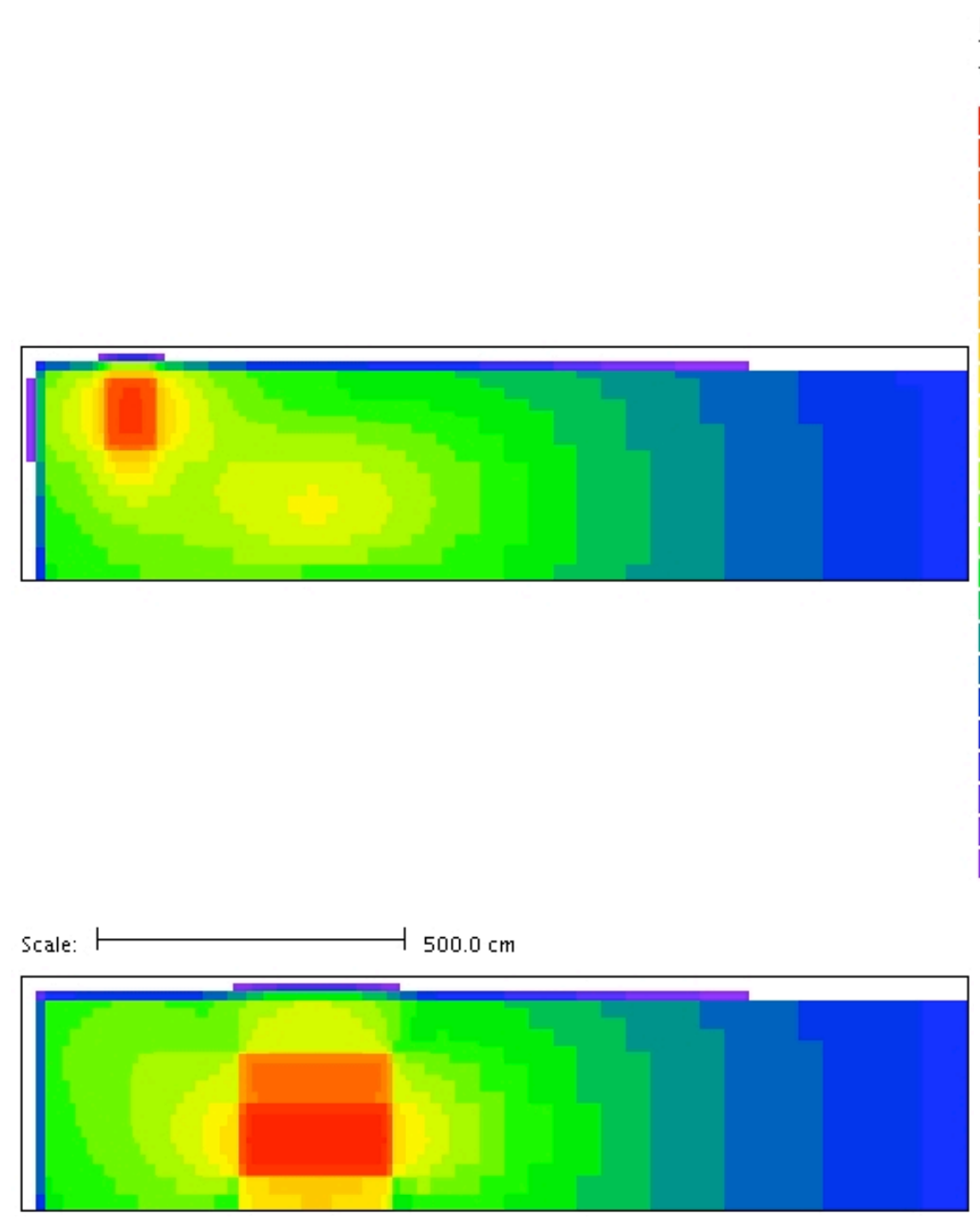

photon Results

Total photon Results

Values

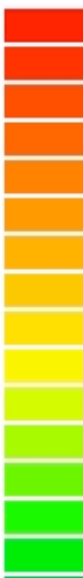

$6.81 \mathrm{E} 01-1.00 \mathrm{E} 02$

$4.64 \mathrm{E} 01-6.81 \mathrm{E} 01$

$3.16 \mathrm{E} 01-4.64 \mathrm{E} 01$

$2.15 \mathrm{E} 01-3.16 \mathrm{E} 01$

1.47E01 - 2.15E01

$1.00 \mathrm{E} 01-1.47 \mathrm{E} 01$

$6.81 \mathrm{E} 00-1.00 \mathrm{E} 01$

$4.64 \mathrm{E} 00-6.81 \mathrm{E} 00$

$3.16 \mathrm{EO0}-4.64 \mathrm{E} 00$

$2.15 \mathrm{E} 00-3.16 \mathrm{E} 00$

$1.47 \mathrm{E} 00-2.15 \mathrm{E} 00$

$1.00 \mathrm{E} 00-1.47 \mathrm{E} 00$

$6.81 \mathrm{E}-01-1.00 \mathrm{E} 00$

4.64E-01 - 6.81E-01

$3.16 \mathrm{E}-01-4.64 \mathrm{E}-01$

$2.15 \mathrm{E}-01-3.16 \mathrm{E}-01$

$1.47 \mathrm{E}-01-2.15 \mathrm{E}-01$

$1.00 \mathrm{E}-01-1.47 \mathrm{E}-01$

$6.81 \mathrm{E}-02-1.00 \mathrm{E}-01$

$4.64 \mathrm{E}-02-6.81 \mathrm{E}-02$

$3.16 \mathrm{E}-02-4.64 \mathrm{E}-02$

$2.15 \mathrm{E}-02-3.16 \mathrm{E}-02$

$1.47 \mathrm{E}-02-2.15 \mathrm{E}-02$

$1.00 \mathrm{E}-02-1.47 \mathrm{E}-02$

Fig. 7. XZ-plane mesh tally dose rate $(\mathrm{mSv} / \mathrm{h})$ contour plots through Y-axis center of filter (top) and ion exchanger (bottom) for barytes concrete walls/ceiling at shutdown. Distance scale and color-coded dose rate values are the same for both plots. 


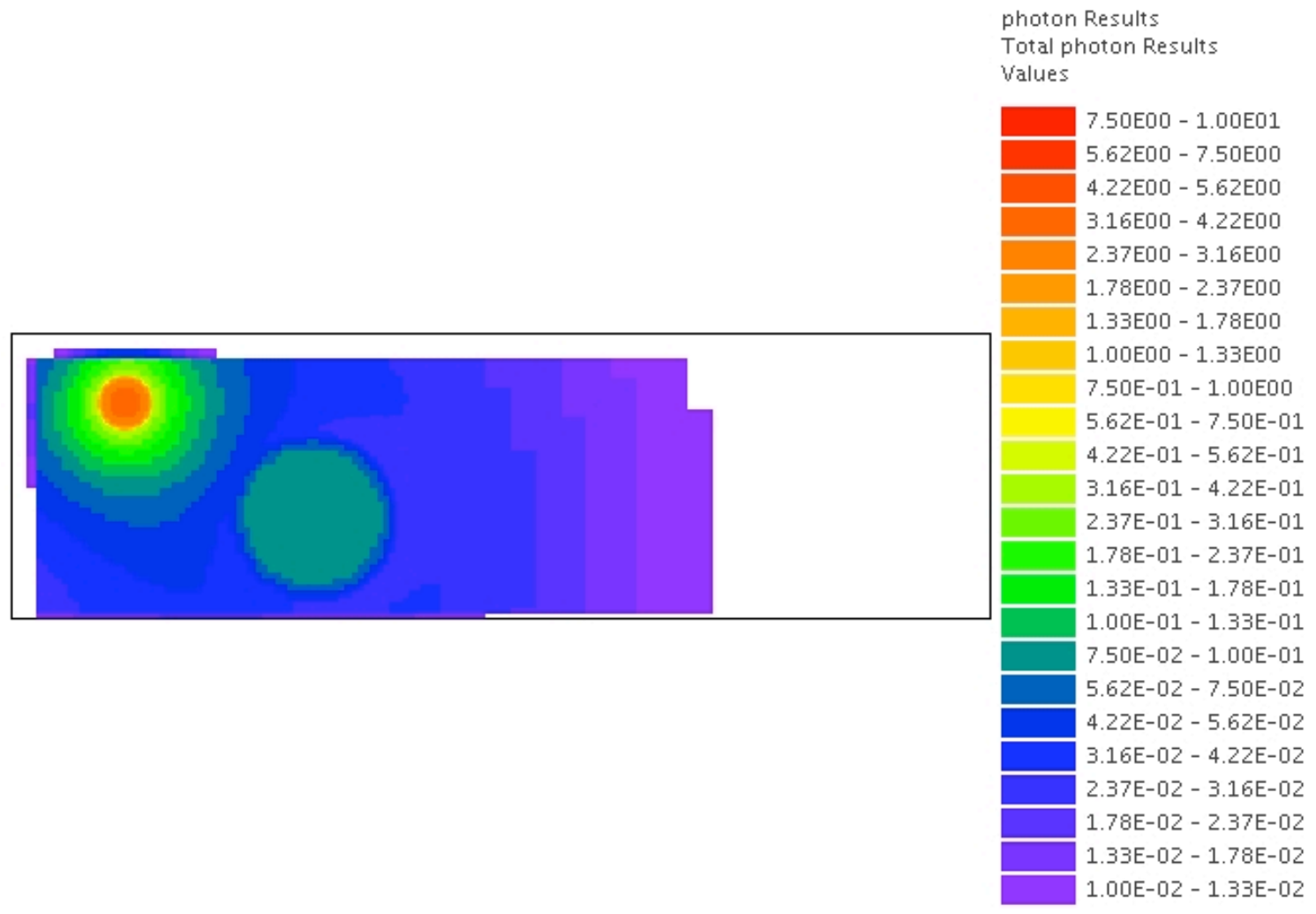

Scale: $\longmapsto 500.0 \mathrm{~cm}$
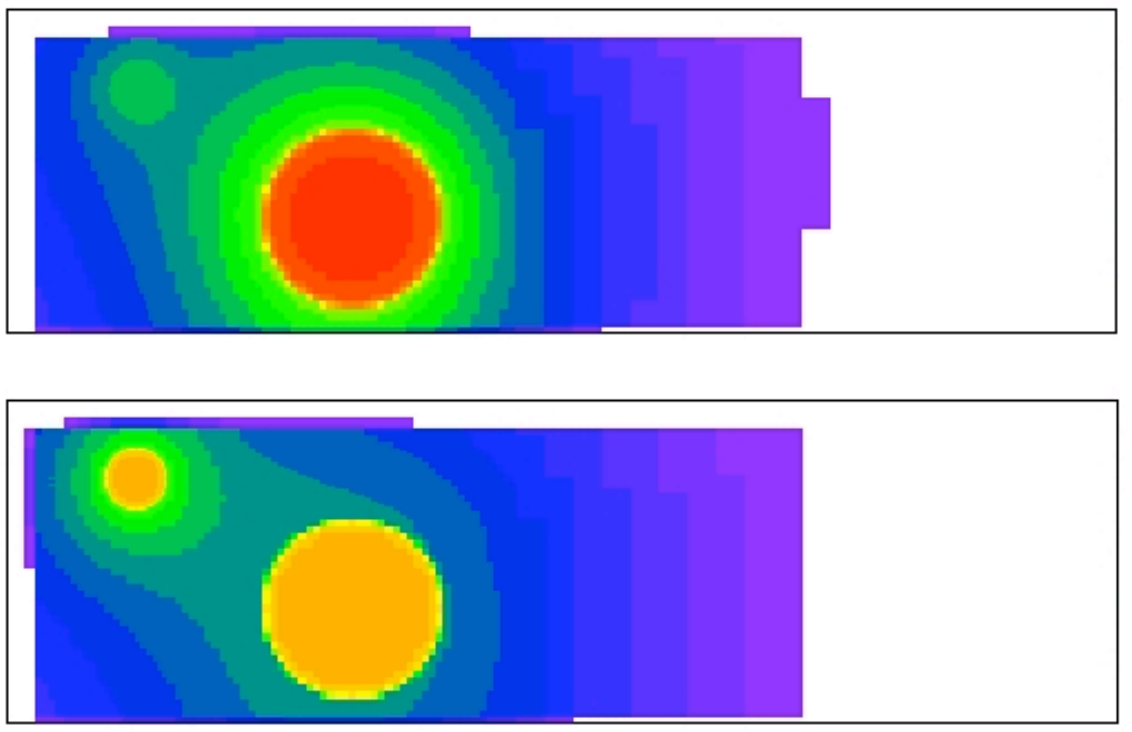

Fig. 8. XY-plane mesh tally dose rate $(\mathrm{mSv} / \mathrm{h})$ contour plots through vertical center of filter (top), ion exchanger resin (center), and ion exchanger water (bottom) for barytes concrete walls/ceiling 2 days after shutdown. Distance scale and color-coded dose rate values are the same for all three plots. 


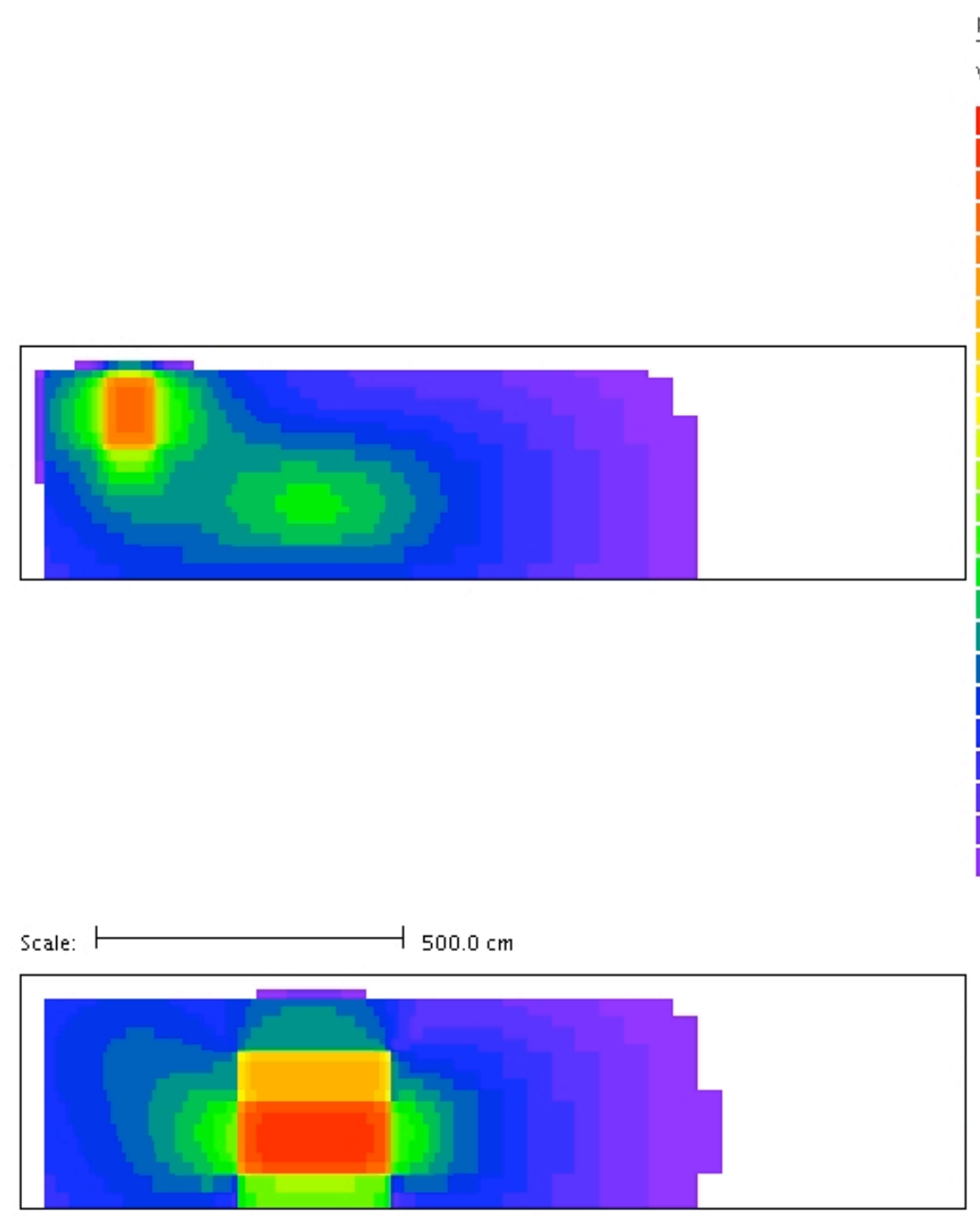

photon Results

Total photon Results

Values

$7.50 E 00-1.00 E 01$

$5.62 \mathrm{E} 00-7.50 \mathrm{E} 00$

$4.22 \mathrm{E} 00-5.62 \mathrm{E} 00$

$3.16 \mathrm{E} 00-4.22 \mathrm{E} 00$

$2.37 \mathrm{E} 00-3.16 \mathrm{E} 00$

$1.78 \mathrm{E} 00-2.37 \mathrm{E} 00$

$1.33 \mathrm{E} 00-1.78 \mathrm{E} 00$

$1.00 E 00-1.33 \mathrm{E} 00$

$7.50 \mathrm{E}-01-1.00 \mathrm{E} 00$

$5.62 \mathrm{E}-01-7.50 \mathrm{E}-01$

4.22E-01 - 5.62E-01

$3.16 \mathrm{E}-01-4.22 \mathrm{E}-01$

2.37E-01 - 3.16E-01

$1.78 \mathrm{E}-01-2.37 \mathrm{E}-01$

$1.33 \mathrm{E}-01-1.78 \mathrm{E}-01$

$1.00 \mathrm{E}-01-1.33 \mathrm{E}-01$

7.50E-02 - 1.00E-01

$5.62 \mathrm{E}-02-7.50 \mathrm{E}-02$

$4.22 \mathrm{E}-02-5.62 \mathrm{E}-02$

$3.16 \mathrm{E}-02-4.22 \mathrm{E}-02$

$2.37 \mathrm{E}-02-3.16 \mathrm{E}-02$

$1.78 \mathrm{E}-02-2.37 \mathrm{E}-02$

$1.33 \mathrm{E}-02-1.78 \mathrm{E}-02$

$1.00 \mathrm{E}-02-1.33 \mathrm{E}-02$

Fig. 9. XZ-plane mesh tally dose rate $(\mathrm{mSv} / \mathrm{h})$ contour plots through $\mathrm{Y}$-axis center of filter (top) and ion exchanger (bottom) for barytes concrete walls/ceiling 2 days after shutdown. Distance scale and color-coded dose rate values are the same for both plots. 


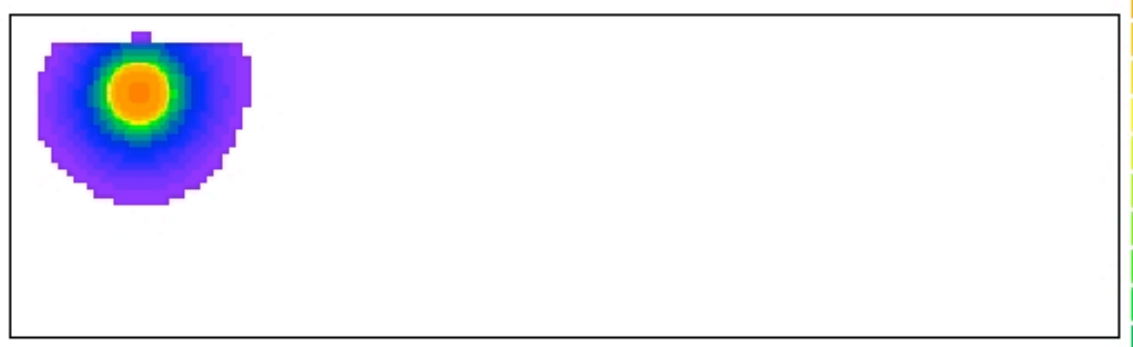

photon Results

Total photon Results

Values

\begin{tabular}{|c|}
\hline $8.25 \mathrm{E}-01-1.00 \mathrm{E} 00$ \\
\hline $6.81 E-01-8.25 E-01$ \\
\hline $5.62 \mathrm{E}-01-6.81 \mathrm{E}-01$ \\
\hline $4.64 \mathrm{E}-01-5.62 \mathrm{E}-01$ \\
\hline $3.83 \mathrm{E}-01-4.64 \mathrm{E}-01$ \\
\hline $3.16 \mathrm{E}-01-3.83 \mathrm{E}-01$ \\
\hline $2.61 \mathrm{E}-01-3.16 \mathrm{E}-01$ \\
\hline $2.15 \mathrm{E}-01-2.61 \mathrm{E}-01$ \\
\hline $1.78 \mathrm{E}-01-2.15 \mathrm{E}-01$ \\
\hline $1.47 \mathrm{E}-01-1.78 \mathrm{E}-01$ \\
\hline $1.21 \mathrm{E}-01-1.47 \mathrm{E}-01$ \\
\hline $1.00 \mathrm{E}-01-1.21 \mathrm{E}-01$ \\
\hline $8.25 \mathrm{E}-02-1.00 \mathrm{E}-01$ \\
\hline $6.81 \mathrm{E}-02-8.25 \mathrm{E}-02$ \\
\hline $5.62 \mathrm{E}-02-6.81 \mathrm{E}-02$ \\
\hline $4.64 \mathrm{E}-02-5.62 \mathrm{E}-0$ \\
\hline $3.83 \mathrm{E}-02-4.64 \mathrm{E}-0$ \\
\hline $3.16 \mathrm{E}-02-3.83 \mathrm{E}-02$ \\
\hline $2.61 \mathrm{E}-02-3.16 \mathrm{E}-0$ \\
\hline $2.15 \mathrm{E}-02-2.61 \mathrm{E}-0$ \\
\hline $1.78 \mathrm{E}-02-2.15 \mathrm{E}-0$ \\
\hline $1.47 \mathrm{E}-02-1.78 \mathrm{E}-0$ \\
\hline $1.21 \mathrm{E}-02-1.47 \mathrm{E}-0$ \\
\hline $1.00 \mathrm{E}-02-1.21 \mathrm{E}-0$ \\
\hline
\end{tabular}

Scale: $\longmapsto 500.0 \mathrm{~cm}$
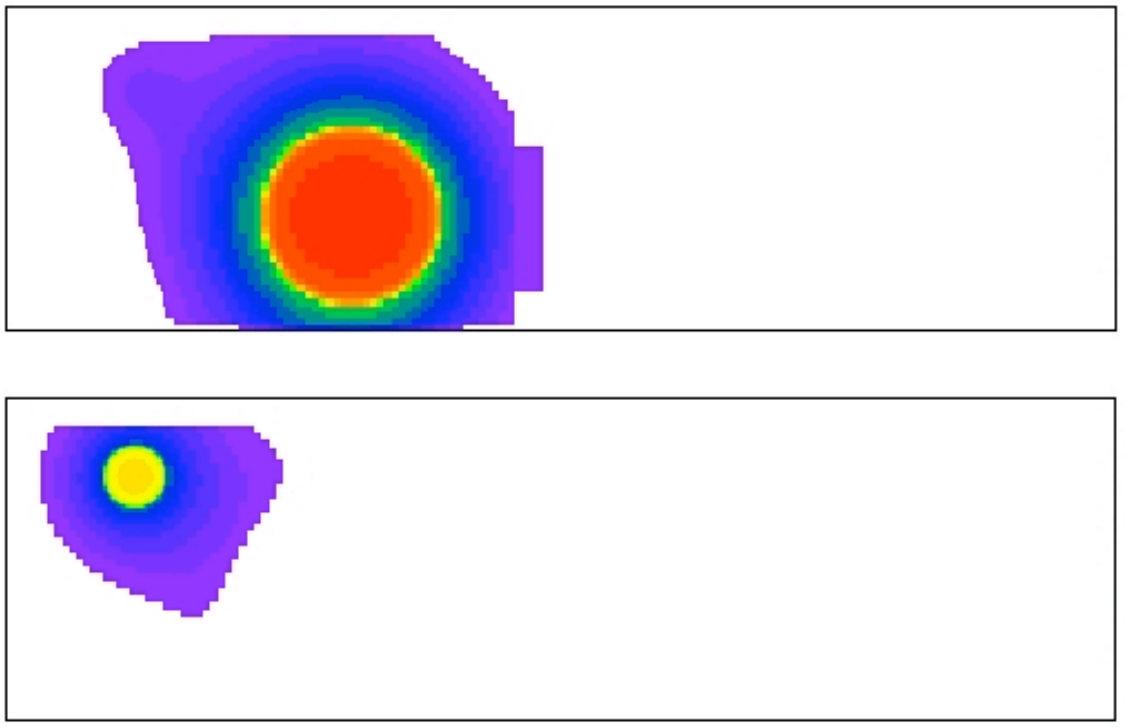

Fig. 10. XY-plane mesh tally dose rate $(\mathrm{mSv} / \mathrm{h})$ contour plots through vertical center of filter (top), ion exchanger resin (center), and ion exchanger water (bottom) for barytes concrete walls/ceiling 10 days after shutdown. Distance scale and color-coded dose rate values are the same for all three plots. 


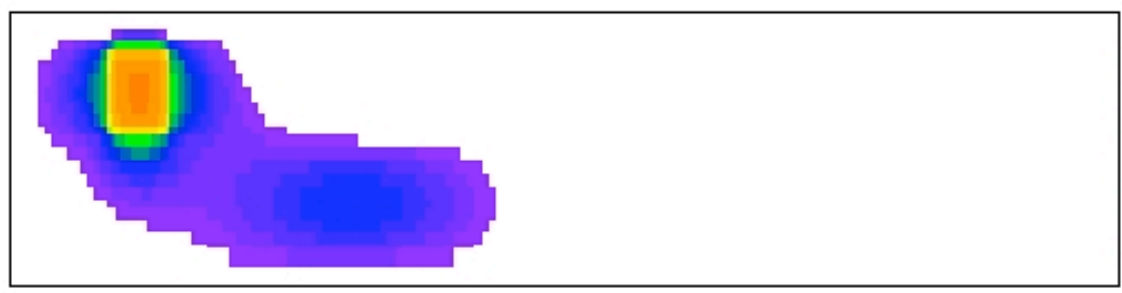

photon Results

Total photon Results

Values

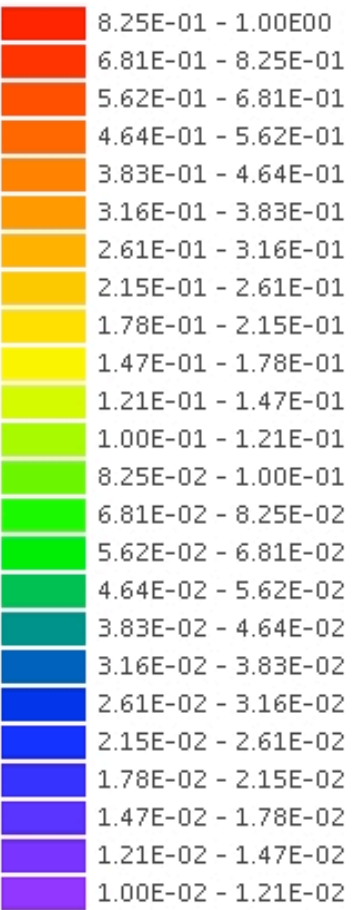

Scale: $\longmapsto 500.0 \mathrm{~cm}$

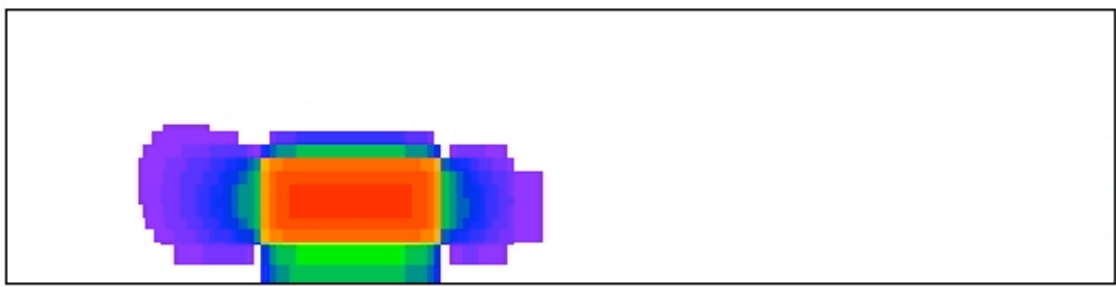

Fig. 11. XZ-plane mesh tally dose rate $(\mathrm{mSv} / \mathrm{h})$ contour plots through Y-axis center of filter (top) and ion exchanger (bottom) for barytes concrete walls/ceiling 10 days after shutdown. Distance scale and color-coded dose rate values are the same for both plots. 


\section{REFERENCES}

1. X-5 Monte Carlo Team. MCNP-A General Monte Carlo N-Particle Transport Code, Version 5, LA-UR-03-1987. LANL, Los Alamos, NM, USA, 2003.

2. V. Barabash and P. Cortes. Fluid Radioactivity Concentration for the ITER Tokamak Cooling Water System. ITER D 26GLXV, v. 2.4. ITER IO, Cadarache, France, 2010.

3. SCALE: A Modular Code System for Performing Standardized Computer Analyses for Licensing Evaluation, ORNL/TM-2005/39. ORNL, Oak Ridge, TN, USA, 2009.

4. J. Berry and A. Petrov. DA Minutes of Meetings October 10 through October 15, 2012. ITER 6PUU2L, v. 1.1. ITER IO, Cadarache, France, 2012.

5. R.G. Williams III, C.J. Gesh, and R.T. Pagh. Compendium of Material Composition Data for Radiation Transport Modeling, PNNL-15870. PNNL, Richland, WA, USA, 2006.

6. National Nuclear Data Center. Brookhaven National Laboratory. Available at http://www.nndc.bnl.gov/. 
US ITER 1020105-TD0001-R00

PA 2.6P1A_B.US.01.0

\section{Appendix}





\title{
Estimation of Activity Accumulated on Inlet Filters and Ion Exchangers of IBED CVCS
}

\author{
Andrei Petrov, TCWS, US ITER
}

\section{Objective}

Estimate the activity on inlet filters and ion exchangers accumulated during operational time between filter/resin replacements. This information is later used for estimation of shielding requirements in the CVCS area.

\section{Problem Description}

The European Union Domestic Agency has decided to accelerate issuance of Tokamak building specifications and requested the information about required shielding in the CVCS area. This estimation supports this request by providing input data to be used in the shielding estimation.

\section{Assumptions}

The approach and models from previously released report [1] with recent estimation of individual ACP isotope activities [2] were used as the basis for estimation of activity around inlet filters and ion exchangers of the IBED CVCS. The basic assumptions used in these estimations were as follows:

3.1 The loop parameters (flowrates, volumes, surface areas, materials, release rates) used in estimation of individual activities [1,2] were the same as those used in previous evaluation of ACPs for the Divertor cooling loop [3]. The flowrate through CVCS was established as $1 \%$.

3.2 The ACP activities on the DIV/LIM inlet filters and ion exchangers were estimated by the difference between the loop activities without and with CVCS over the operational period of 1.5 years.

3.3 The ACP activities on the IBED inlet filters and ion exchangers were estimated by multiplying corresponding DIV/LIM activities by 4 .

3.4 The following ACP isotopes were considered in this estimation [3, 4]: Fe-59, Mn-54, Fe-55, Co-58, Co-60, Cr-51, Mn-56, and Cu-64.

3.5 The ACP removal efficiency was established as follows: $98 \%$ for all isotopes (except $\mathrm{Cu}-64$ ) and $70 \%$ for $\mathrm{Cu}-64$.

3.6 It was assumed that $90 \%$ of the total mass release was in soluble form, and $10 \%$ - in insoluble form [5].

3.7 It was assumed that $8 \%$ of the total mass release was deposited on the loop surfaces [3]; although for activity and dose rate estimations, more conservative data without account for deposition were used (Tables 1 and 2).

3.8 The surface areas of IBED inlet filter and ion exchanger were estimated from the cut-out sheets provided by AREVA. 


\section{Approach}

The total activities of the radionuclides of interest in the Diverter loop after 1.5 years of operation - with and without purification - are shown in Table 1 [1]. These data were used, in accordance with the above assumptions, to estimate source terms associated with IBED inlet filter and ion exchanger (Table 2).

Table 1. Total Radionuclide Activities (Bq) after 4,357 Cycles (1.5 years of Operation) [1]

\begin{tabular}{|l|c|c|}
\hline \multicolumn{1}{|c|}{ Radionuclide } & No Filtration/Ion Exchange & 1\% Filtration/Ion Exchange \\
\hline Cu-64 & $2.53 \mathrm{E}+12$ & $5.88 \mathrm{E}+11$ \\
\hline Mn-56 & $9.03 \mathrm{E}+10$ & $4.37 \mathrm{E}+10$ \\
\hline Cr-51 & $4.95 \mathrm{E}+09$ & $4.31 \mathrm{E}+07$ \\
\hline Co-58 & $1.43 \mathrm{E}+09$ & $5.88 \mathrm{E}+06$ \\
\hline Mn-54 & $9.44 \mathrm{E}+08$ & $2.07 \mathrm{E}+06$ \\
\hline Fe-55 & $7.83 \mathrm{E}+08$ & $1.47 \mathrm{E}+06$ \\
\hline Co-60 & $7.69 \mathrm{E}+08$ & $1.40 \mathrm{E}+06$ \\
\hline Fe-59 & $1.48 \mathrm{E}+08$ & $8.65 \mathrm{E}+05$ \\
\hline
\end{tabular}

Table 2. Source Terms Associated with IBED Inlet Filter and Ion Exchanger after 4,357 Cycles (1.5 years of Operation)

\begin{tabular}{|l|c|c|c|c|c|c|c|c|c|}
\hline & \multicolumn{9}{|c|}{ Isotopes, GBq } \\
\hline & Fe-59 & Mn-54 & Fe-55 & Co-58 & Co-60 & Cr-51 & Mn-56 & Cu-64 & Total \\
\hline IBED Filter & 0.05 & 0.34 & 0.16 & 0.52 & 0.28 & 1.78 & 16.94 & 708.06 & 728.13 \\
\hline $\begin{array}{l}\text { IBED Ion } \\
\text { Exchanger }\end{array}$ & 0.54 & 3.43 & 2.96 & 5.18 & 2.79 & 17.84 & 169.37 & 7080.58 & 7282.69 \\
\hline
\end{tabular}

These data were used as inputs to the estimation of the shielding around filters and ion exchangers in the CVCS area. 


\section{References}

1. O. Jonas and L. Machemer. Corrosion, Activation, Water Chemistry, and MaterialsITER Tokamak Cooling Water Systems - Interim Assessment. U.S. ITER Task 4 Report. U.S. ITER, ORNL, Oak Ridge, TN, USA, 2008.

2. L. Machemer. Estimation of ACP Isotope Activities for Copper-Based TCWS Loop during ITER Operation. U.S. ITER, ORNL, Oak Ridge, TN, USA, 2011.

3. L. Di Pace. Activated Corrosion Products Evaluation for the ITER TCWS DIV/LIM Loop Using the PACTITER $V$ 2.1. EFDA Task TW-TSS-SEA5 Final Report. ENEA, Frascati, Italy, 2003.

4. Preliminary Safety Report. Volumes I and II. ITER 3ZR2NC. ITER IO, Cadarache, France, 2010.

5. D. Hussey. Impacts of CVCS Cleanup Systems on Activity Release and Dose Rate. Proceedings of the 2007 Condensate Polishing Workshop, June 5-7, 2007, Palm Springs, CA. EPRI Report 1015447. EPRI, Palo Alto, CA, USA, 2007. 